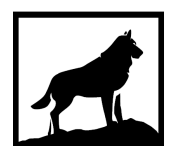

Michigan Technological

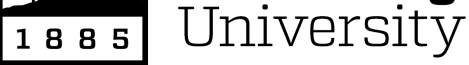

Michigan Technological University Digital Commons @ Michigan Tech

2018

EMPLOYING DIELECTRIC-BASED MEDIA FOR CONTROLLING

FIELD PATTERNS AND WAVE PROPAGATION IN ADVANCED ELECTROMAGNETIC DEVICES

Navid Pourramzan Gandji

Michigan Technological University, npourram@mtu.edu

Copyright 2018 Navid Pourramzan Gandji

Recommended Citation

Pourramzan Gandji, Navid, "EMPLOYING DIELECTRIC-BASED MEDIA FOR CONTROLLING FIELD PATTERNS AND WAVE PROPAGATION IN ADVANCED ELECTROMAGNETIC DEVICES", Open AccesS Dissertation, Michigan Technological University, 2018.

https://doi.org/10.37099/mtu.dc.etdr/677

Follow this and additional works at: https://digitalcommons.mtu.edu/etdr

Part of the Electrical and Computer Engineering Commons 


\title{
EMPLOYING DIELECTRIC-BASED MEDIA FOR CONTROLLING FIELD PATTERNS AND WAVE PROPAGATION IN ADVANCED ELECTROMAGNETIC DEVICES
}

\author{
By: \\ Navid Pourramzan Gandji
}

\begin{abstract}
A DISSERTATION
Submitted in partial fulfillment of the requirements for the degree of DOCTOR OF PHILOSOPHY In Electrical Engineering MICHIGAN TECHNOLOGICAL UNIVERSITY 2018 (C) 2018 Navid Pourramzan Gandji
\end{abstract}


This dissertation has been approved in partial fulfillment of the requirements for the Degree of DOCTOR OF PHILOSOPHY in Electrical Engineering.

Department of Electrical \& Computer Engineering

Dissertation Advisor: Elena Semouchkina

Committee Member: Smitha Malalur Nagaraja Rao

Committee Member: $\quad$ Durdu Guney

Committee Member: Warren F. Perger

Department Chair: Daniel R. Fuhrmann 


\section{Contents}

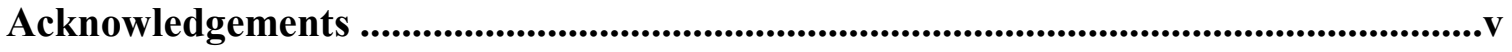

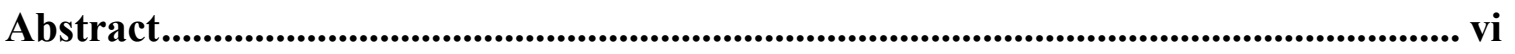

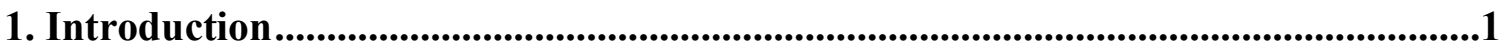

1.1 Dielectric materials and recent advances in their employment ............................1

1.2 RF probes for ultrahigh field MRI scanners ......................................................

1.3 Mie resonances in dielectric particles and negative refraction .............................

1.4 Directional wave scattering from metasurfaces of dielectric nano-disk resonators

1.5 All-dielectric photonic crystals for transformation optics based devices ............11

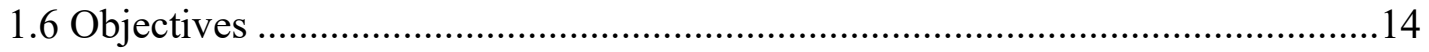

2. Developing and Experimental Testing of alternative RF probes for 14T MRI

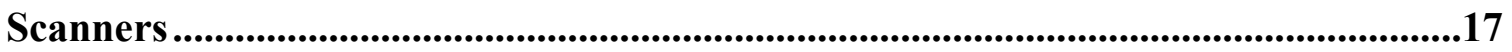

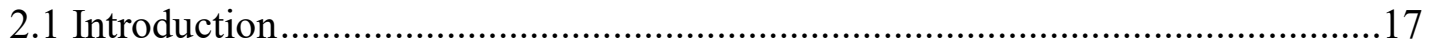

2.2 Patch with Engineered Multi-Dielectric Substrate as the Surface Probe..............17

2.3 System of vis-à-vis Placed Patches as Volume Probe..........................................24

2.4 Systems of Profiled Patches as Volume Probes ..................................................28

2.5 Comparing Patch Probes with Birdcage Coils ........................................................33

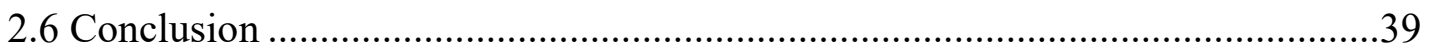

3. Clarifying the nature of negative refraction in all-dielectric metamaterials composed of identical resonators...............................................................................................41

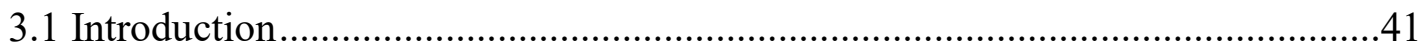

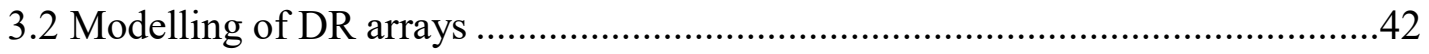

3.3 Mie resonances versus surface resonances in rod arrays .....................................4

3.4 Correlation between changes of index spectra and of dispersion diagrams at decreasing the lattice constant of arrays ..................................................................4

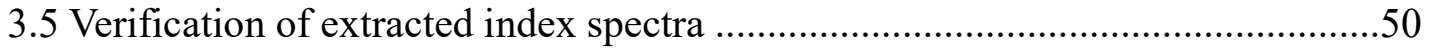

3.6 Applicability of the conclusion about the nature of negative refraction to other DR

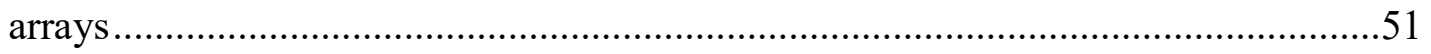

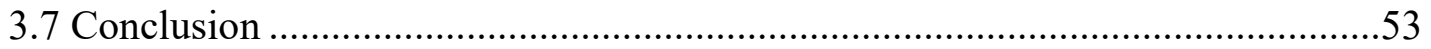


4. Electromagnetic responses of metasurfaces composed from dielectric nano-disk resonators....................................................................................................................................54

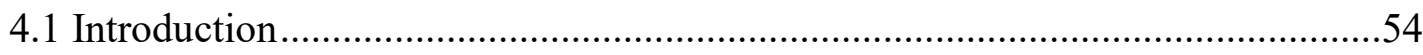

4.2 Changes in spectral positions of dipolar resonances in nano-disks at varying

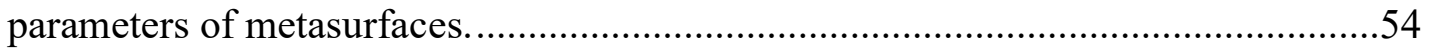

4.3 Correlation between EM responses of metasurfaces and dispersion phenomena in

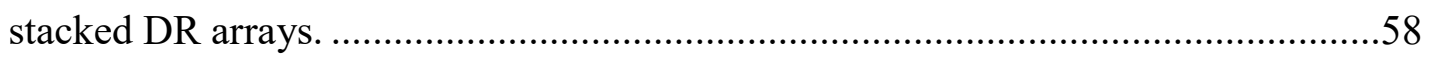

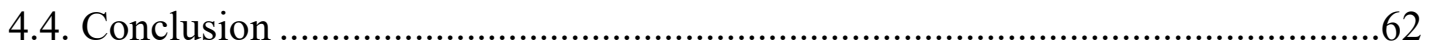

5. Selected problems of implementing all-dielectric photonic crystals in the media of transformation optics based devices.........................................................................................63

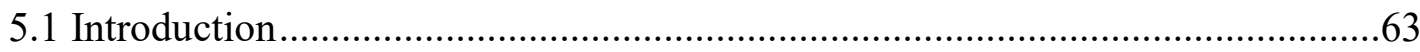

5.2 $\mathrm{PhC}$ parameters providing superluminal wave propagation .................................64

5.3 Bending the wave paths in cylindrical cloak composed of rod arrays..................66

5.4 Optimal structure of PhC fragments and clarifying the problem of EM interaction

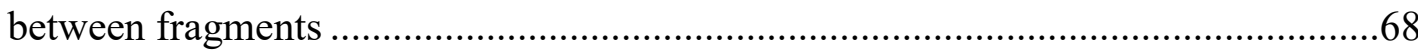

5.5 Additional options for employing the self-collimation in cloaking devices .........71

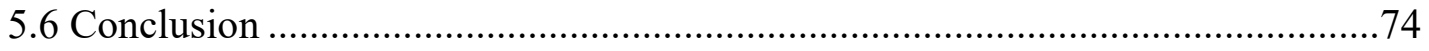

6. Summary and future works .........................................................................................75

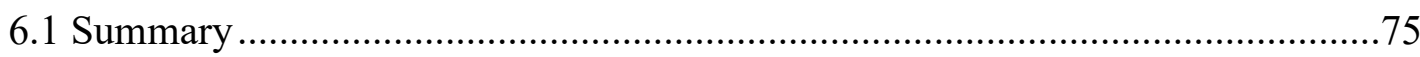

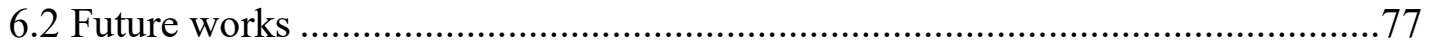

References..........................................................................................................................79

Appendix A. Copyright permission of the figures in chapter 3 .....................................85

Appendix B. Copyright permission of the figures 5.4 and 5.5 .......................................86

Appendix C. Copyright permission of the figures 5.6 and 5.7 …….................................87 


\section{Acknowledgements}

I would like to extend my thanks to the many people, without the support of whom, My $\mathrm{PhD}$ study would not have been possible to be accomplished.

The first and most special thanks go to my supportive supervisor, Prof. Elena Semouchkina, not only for her great academic support, but also for trusting and giving me so many wonderful opportunities.

Secondly, I want to give my tremendous thanks to my committee members Dr. Smitha Rao, Dr. Durdu Guney, and Dr. Warren Perger for willing to serve on my dissertation committee, spending time to read my dissertation and providing valuable comments for me.

Thirdly, I thank my group members in our research lab: Dr. George Semouchkin, Dr. Xiaohui Wang, Dr. Fang Chen, Dr. Ran Duan, and Saeed Jamilan who accompanied me in this journey and kept me on my toes.

I am very grateful to Dr. Michael Lanagan, Dr. Thomas Neuberger and Dr. Gangchea Lee from Pennsylvania State University, for their collaboration in some parts of this dissertation.

I would like to thank also Electrical and Computer Engineering Department in Michigan Technological University from chair to staff who helped me to continue my research.

The final thanks belong to my parents and family members, for their unconditional love and support. Most importantly, I wish to thank my beautiful wife, Atefeh, for providing unending inspiration. 


\begin{abstract}
Rapid progress in developing electromagnetic devices and in governing the wave propagation during last years caused renewed interest to dielectric materials. First, engineered dielectric structures with spatial dispersion of their parameters came to replace uniform substrates in antennas and other resonance devices. Then additional boom of dielectric applications was caused by the possibility to employ dielectrics as materials of artificial media. Later, attention of researchers was attracted to properties of the media composed of dielectric resonators (DRs). Currently DRs are used to create metamaterials - the media with unprecedented properties, which cannot be found in nature. Dielectric photonic crystals and metamaterials are considered as the most perspective materials for photonics, since they can be integrated in devices without loss problems, which characterize, for example, plasmonic techniques. Recently, a booming interest emerged to employing in photonics directional light scattering from dielectric particles, since the wavelengths of this light could be controlled by dimensions of particles and their dielectric permittivity.

Our work followed basic innovations, which defined contemporary employment of dielectrics in electromagnetics and photonics. In particular, we started from working out new engineering approaches to developing dielectric substrates in patch structures inspired by microstrip patch antennas, which are proposed to serve as MRI RF probes (Chapter 2). Then we redirected our attention to the problems, which restricted employment of dielectrics in left-handed media. In particular, we have shown that negative refraction in all-dielectric metamaterials is irrelevant to Mie resonances in dielectric elements (Chapter 3). Next, we turned to analysis of problems defining directional scattering from dielectric metasurfaces and have demonstrated that the nature of observed phenomena cannot be correctly understood without accounting for strong interaction between "atoms" of metasurafces (Chapter 4). Finally we discussed selected problems met at implementation of photonic crystals in the media of transformation optics based devices and have shown that some of the problems can be solved at employing the phenomenon of self-collimation, characteristic for periodic photonic structures (Chapter 5).
\end{abstract}




\section{Introduction}

\subsection{Dielectric materials and recent advances in their employment}

Dielectric materials have been conventionally considered as materials in which negatively charged electrons are firmly bound to positively charged nucleus. This defined their applications as insulators well described in electrostatics theory. According to this theory, external electric field applied to dielectric media should cause a force to be exerted on each charged particle that results in displacement of positive and negative charges in opposite directions (Figure 1.1). This displacement (even small compared to the atomic dimension) polarizes the dielectric material that decreases its internal field and can be described by increased compared to vacuum as relative dielectric permittivity. This property of dielectric media is employed in capacitors for energy storage [1].

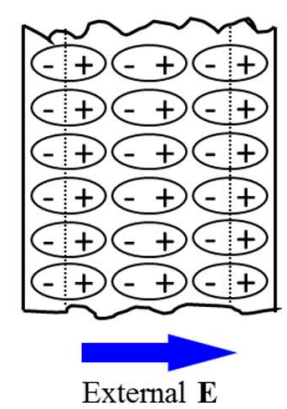

Figure 1.1 A schematic cross section of polarized dielectric medium

The polarization in the dielectric material can be presented by the following equation:

$$
\overrightarrow{P_{e}}=\varepsilon_{0} \chi_{e} \vec{E}
$$

where $\varepsilon_{0}$ is the permittivity of the free space $\left(\varepsilon_{0} \approx 8.85 \times 10^{-12}\right), \chi_{e}$ is the electric susceptibility of the material, and $\vec{E}$ is the electric field. The electric flux density (or electric displacement), $\mathrm{D}$, is then defined as

$$
\begin{aligned}
\vec{D} & =\varepsilon_{0} \vec{E}+\overrightarrow{P_{e}} \\
& =\varepsilon_{0}\left(1+\chi_{e}\right) \vec{E}=\varepsilon_{0} \varepsilon_{r} \vec{E}=\varepsilon \vec{E}
\end{aligned}
$$

where $\varepsilon$ is the absolute permittivity (also called as "permittivity") and $\varepsilon_{r}$ is named as the relative permittivity or dielectric constant of the material [2], and generally is a complex number. Values of $\varepsilon_{r}$ range from 1 to 10 for many plastics, 10 to 100 for polar solvents such 
as water $\left(\varepsilon_{r}=81\right), 100$ to several thousand for some compounds such as $\operatorname{SrTiO} 3\left(\varepsilon_{r}=300\right)$ or BST $\left(\varepsilon_{r}=600\right)$, and above 10,000 for specialized materials which exhibit what is known as "giant permittivity" materials [3].

The effect of magnetic field on dielectric materials has been usually considered as insignificant. Although orbiting around nucleus electrons can be considered as circulating current creating magnetic dipoles, their contribution in magnetization (magnetic analogue of polarization) is negligible and does not lead to any meaningful difference of dielectric permeability from characteristic for vacuum value $\mu_{0}=4 \pi \times 10^{-7} \mathrm{H} / \mathrm{m}$.

Relatively simple and basically linear character of responses to applied fields from conventional dielectric materials was the reason of low scientific interest to dielectrics during many years. However, recently the situation experienced drastic changes. First, dielectrics were found to be best materials for building photonic crystals - artificial materials capable of controlling propagation of EM waves similar to that provided by natural crystals in optics [4-6]. Then, in 1999, a new class of artificial materials with unprecedented properties has been discovered [7]. They were called Metamaterials (from the Greek word $\mu \varepsilon \tau \alpha$ (meta), which means "beyond"), and were shown to perform due to electric and magnetic resonances in their "atoms" [8]. Originally, MMs were composed of metallic resonators. However, soon it became clear that metamaterials can be created also by using dielectric resonators $[9,10]$. In particular, it was revealed that the capability of dielectric resonators to support magnetic resonances can transform arrays of dielectric resonators into the media with relatively high magnetic permeability and that such media can demonstrate as positive, so negative refraction $[9,10]$. Thus, arrays of dielectric resonators were found able to support backward wave propagation [11]. All-dielectric photonic crystals, in turn, were found capable of competing with metamaterials in providing unusual properties, which seemed rather impossible for natural materials and were called the "left-handedness" $[12,13]$. In addition, both classes of all-dielectric media demonstrated low loss and promised unrestricted employing in high frequency ranges, up to optics. Therefore, about 5 years ago interest of front-edge researchers all over the world started to shift to nano-photonics based on dielectric media, instead of searching low loss plasmonic solutions $[14,15]$. Presented below work is aimed at participating in the 
described above development and, although it started from employing conventional approaches for engineering dielectric media in patch structures (Chapter 2), then became redirected to problems restricting employment of left-handed all-dielectric media in various conditions, including those in nano-photonics (Chapters 3 and 4) and in transformation optics based devices (Chapter 5).

It is worth noting that, since new applications of dielectric media are basically defined by the effects of media properties on wave propagation, it is important to realize how these properties affects the solutions of Maxwell equations. The "wave equation", used to describe the EM wave propagation in dielectric media has been derived from Maxwell's equation, and by assuming the sinusoidal EM wave, could be written in form [16]:

$$
\nabla^{2} \vec{\psi}+k^{2} \vec{\psi}=0, \quad k^{2}=\left(\frac{\omega}{c}\right)^{2} \varepsilon_{r} \mu_{r}
$$

Where $\vec{\psi}$ is either E- or H-field vector, $\mathrm{k}$ is the propagation constant, $\omega$ is the angular frequency and $c$ is the speed of light in free space. The general solution for Equation 1.4 is

$$
\vec{\psi}=\overrightarrow{\psi_{0}} e^{ \pm j \vec{k} \cdot \vec{r}}
$$

where $\vec{k}$ vector is directed along the wave propagation and $\vec{r}$ is the radius-vector. The wavelength inside dielectric materials is inversely proportional to the refractive index, which is defined as $n=\sqrt{\varepsilon_{r} \mu_{r}}$.

While the relative permittivity and permeability of a conventional dielectric material are always the same within the chosen medium, metamaterials and photonic crystals with, characteristic for them, unconventional EM properties could be used to change wave propagation locally and make it functional as it takes place in transformation optics based devices [17]. The dielectric media, properties of which depend on periodicity, as in photonic crystals, are well investigated theoretically and can be described by using different approximations for solving Maxwell and Bloch-Floquet equations. In particular, they can be represented by dispersion diagrams [18], which characterize propagation bands and also the band gaps, defining the ranges of frequencies, at which wave transmission 
through the medium is excluded. In addition, at the analysis of photonic crystals, researchers often use the equi-frequency contours (EFCs) [19].

In difference from photonic crystals, metamaterials are usually described in the frame of so-called effective medium theory, which ignores periodicity and considers the material to be totally homogenized, when all unit cells respond identically [20-23]. This approach is in some sense close to the approach used by Clausius-Mossotti at consideration of dielectric polarization [24]. It let to the suggestion that the theory should work, if the size of each unit cell of metamaterial is much smaller than the wavelength in free space; therefore, "waves do not see the details of structure" [25]. In such cases, the media can be presented by effective permittivity $\left(\varepsilon_{e f f}\right)$ and permeability $\left(\mu_{e f f}\right)$. The resonance responses, which define the values of the above parameters, are supposed to obey the Lorentz approximation with definitions of frequency ranges with positive and negative values of parameters [7]. In this dissertation, we investigate and use various dielectric media with the aim to provide advanced functionalities of selected EM devices and address challenges preventing them from proper development and employing. Below we briefly present chosen topics and characterize the problems, solutions of which has requested the conducted work.

\subsection{RF probes for ultrahigh field MRI scanners}

Magnetic resonance imaging (MRI) is a noninvasive tool for imaging a comprehensive range of biological tissues from single cells to humans [26-29]. Basic components of MRI systems as shown in Figure 1.2 are:

- Super conducting magnet,

- Gradient coil,

- Radio frequency (RF) coil/probe. 


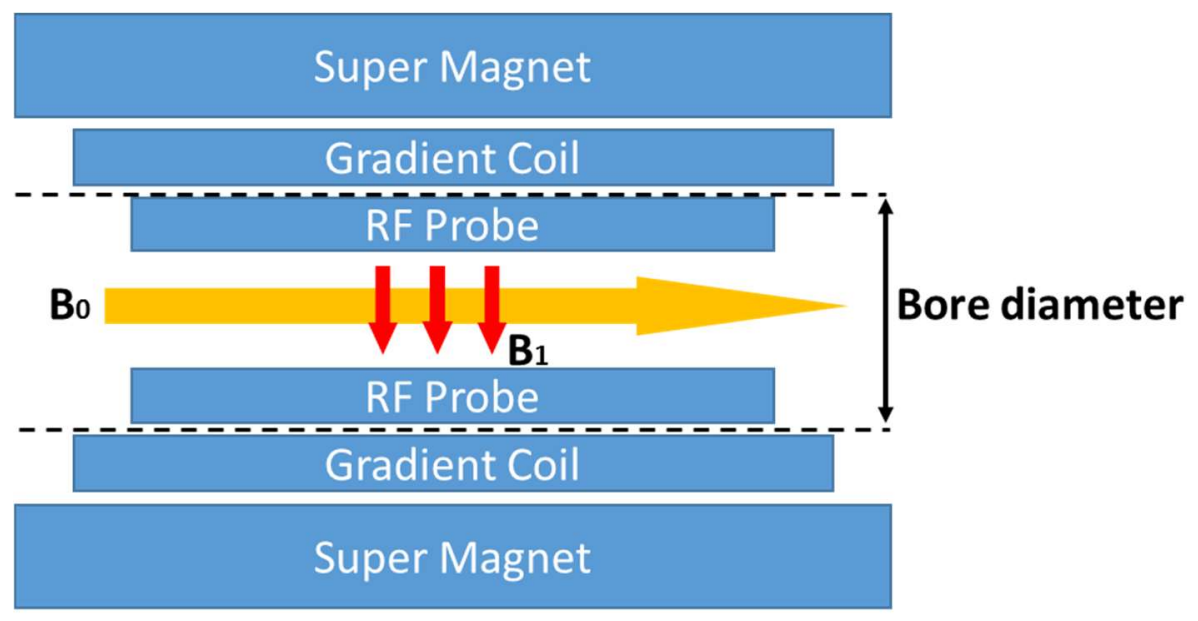

Figure 1.2 Cross-sectional schematic of basic components for each MRI system.

Super conducting magnet in the MRI system supplies a large static magnetic field, $\mathrm{B}_{0}$, along the bore of MRI system (Figure 1.2) and pushes quantum magnetic moments of protons/nuclei in the sample to become parallel the axis of bore (direction of $\mathrm{B}_{0}$ ). The Gradient coil is used to produce deliberate spatial variation in $\mathrm{B}_{0}$, and this variation permits the localization of image slices. Radio Frequency (RF) coil/probe, as another essential component of MRI systems, serves as the transmitter/receiver and creates an alternating magnetic field (known as $\mathrm{B}_{1}$ ) normal to $\mathrm{B}_{0}$. The frequency of $\mathrm{B}_{1}$ is linearly proportional to $\mathrm{B}_{0}$ following the Larmour equation, $\omega=\gamma B_{0}$, where $\gamma$ is called as the gyromagnetic ratio and is equal to $42.53 \mathrm{MHz} / \mathrm{T}$ for hydrogen nuclei [30]. The imaging data originate from responses of tissues under study to RF pulses of $\mathrm{B}_{1}$ magnetic field.

Conventionally, various types of coils have been used as RF probes for creating $\mathrm{B}_{1}$ fields. Obtaining high resolution images in MRI scanners requires high signal-to-noise ratio (SNR) in probe responses [31]. Achieving higher SNR values is possible at higher strength of $\mathrm{B}_{0}$ field [32]. Therefore, soon after MRI invention for human studies in early $1970 \mathrm{~s}$, the call to improve the resolution of imaging has requested progressive increase in the strength of $\mathrm{B}_{0}$ fields, from fractions of one Tesla (1T) in the 1980s, up to $3 \mathrm{~T}$ by the late 1990s [33]. Currently, MRI scanners with $\mathrm{B}_{0}$ up to $7 \mathrm{~T}$ are in ordinary use for clinical human studies. For research purposes, ultra-high fields (UHF) $\left(\mathrm{B}_{0}>7 \mathrm{~T}\right)$ up to $10.5 \mathrm{~T}$ are also used $[34,35]$, while for animal studies, scanners with $\mathrm{B}_{0}$ fields up to $21.1 \mathrm{~T}$ are applied [36-38]. At increasing the strength of static fields, the frequency of B1 fields should also grow up 
following previously mentioned Larmour relation. Therefore, the wavelengths used for imaging become shorter in UHF MRI systems, especially within samples of high dielectric permittivity, that can cause inhomogeneity of B1 field distributions [39] and decrease of the field of view (FOV) (the area or volume over which an MR image is acquired). Therefore, special care about homogeneity of $\mathrm{B}_{1}$ field distribution presents a critical problem for UHF MRI probes.

RF probes could be divided into two categories: surface probes and volume probes [40]. Surface probes are typically employed for imaging topical tissues [41, 42]. However, at high frequencies, conventional surface coils suffer from low transmitting/receiving efficiency because of attenuating $B_{1}$ field in conducting layers of tissues [43]. To increase the SNR, it was proposed to employ dipole antennas instead of coils as RF probes [44, 45]. Other alternatives to surface coils included folded dipole array [46], monopole [47] and slot antennas [48]. However, operating with antennas in UHF MRI systems is complicated by small sizes of bores [49]. For example, the bore of 14T MRI scanner has typically the diameter of $54 \mathrm{~mm}$. Therefore, antennas should be properly miniaturized to be used as RF probes inside MRI bores.

Most common types of volume coils, which should create $\mathrm{B}_{1}$ field within extended volumes, such as human/animals heads or bodies, are birdcage coils and TEM coils [5053]. In order to provide homogeneous distributions of $B_{1}$ field in desired volumes, their designs often require substantial complications. One of the problems with these coils is that they begin to resonate at adjusting to scanners with bores of small sizes. In addition, there are problems with their impedance matching and with decreasing of their quality $(\mathrm{Q})$ factors at placing coils inside MRI bores. As known, the Q-factor, is tied to SNR. To realize high-Q probes to provide high SNR, the use of dielectric resonators (DRs) has been suggested in $[54,55]$. However, because of problems with excitation, DR probes have been used only for very small samples such as zebra fish. To resolve the problem of B1 uniformity in large volumes, authors of [56-58] proposed to employ travelling waves within the bores, which could perform as waveguides. However, small bores of UHF MRI scanners, operating at higher frequencies could not be used as waveguides because of supporting only evanescent waves. 
In this dissertation, we develop approaches to designing both surface and volume RF probes for UHF scanners by employing multi-layer structures originating from the designs of microstrip patch antennas (MPAs). We will call further these structures as "patches" even though in some cases they will have a little in common with typical MPAs. As known, performance of MPAs is based on resonant wave oscillations in the cavities formed in substrates between patch and ground plane electrodes. These oscillations are accompanied by currents in the patch, and, respectively, by oscillating magnetic fields created around patches. Since currents are directed along the length of the patch, magnetic fields should act along its width, i.e. to be normal to the direction of currents that provides convenient opportunity for employing respective fields as testing $\mathrm{B}_{1}$ fields in MRI systems. Patch probes promise a high Q-factor because of their resonance nature, and a possibility to provide high uniformity of magnetic field along the width of planar MPAs-type structure. However, it is worth noticing here that designing patch probes pursues opposite target in comparison with designing MPAs. In particular, patch probes should not be good radiators to avoid strong radiation losses.

It will be shown in Chapter 2 that single patch probes can perform as surface coils, while properly organized systems of coupled patches can provide 3D sampling volumes with controlled uniformity of magnetic field in the space between patches. It is important to underline once again here that despite close to MPA designs, patches used in MRI scanners are not supposed to act as radiation sources and perform, instead, as antenna-inspired resonators, which create, similar to RF coils, magnetic fields necessary for testing the samples under study. While RF coils start to suffer from accompanying resonances, which disturb formation of $\mathrm{B}_{1}$ fields even at $600 \mathrm{MHz}$ [59], RF probes made of patch structures tend to employ resonances for producing strong magnetic fields at frequencies up to microwave range. In order to fit patch probes in small MRI bores, we miniaturized them either by embedding in their low permittivity substrates several high permittivity pellets $[60,61]$ or by using in their design bulk substrates of higher permittivity. 


\subsection{Mie resonances in dielectric particles and negative refraction}

As it was already mentioned above, significant advances in employing dielectric materials in electromagnetics were defined by the revealed opportunity to form, from dielectric particles, the media with properties characteristic for MMs. The most promising property of MMs is known to be the negative refraction supposedly caused by the double negativity of effective parameters - permittivity $\varepsilon_{\text {eff, }}$ and permeability $\mu_{\text {eff. }}$. The investigation of negative refraction and other left-handed phenomena was pioneered by Victor Veselago in 1968 when he studied substances with simultaneously negative effective permittivity, $\varepsilon_{\text {eff, }}$ and permeability, $\mu_{e f f}[62]$. However, until 2000 researchers did not see an opportunity for obtaining the left-handedness in practice. According to first reports about realizing the lefthanded media the double negativity could be achieved at simultaneous excitation of two types of dipolar resonances - electric and magnetic [63, 64]. Pendry, Smith et al. have proposed to excite the electric resonance in cut wires and the magnetic resonance - in splitring resonators $[65,66]$. The frequencies of two resonances had to be properly adjusted to provide the negativity of effective parameters in composed medium - permittivity and permeability - in overlapping frequency ranges. Figure 1.3(a) and (b) presents an opportunity to compare the refractions of incident beam at passing the prism with double positive and double negative parameters.

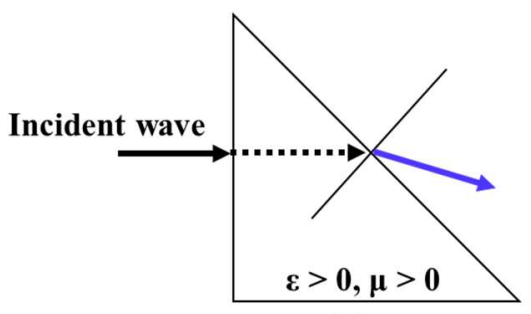

(a)

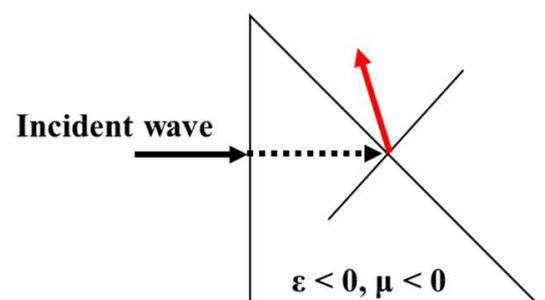

(b)

Figure 1.3 the incident beam refraction from the prism with simultaneously (a) positive and (b) negative constitutive parameters.

In all-dielectric MMs the magnetic resonance could be excited by displacement currents induced in dielectric resonators by magnetic fields of incident waves [67]. The electric resonance could be produced by oscillations of electric fields of incident waves due to reflections of waves from resonator boundaries [68]. Originally, it was supposed 
worthwhile of employing two different types of resonators for exciting two resonances, i.e. to act similar to that at designing conventional MMs [69, 70]. However, later it was suggested that two resonances can be excited in the same resonators and, thus, all-dielectric MMs can be composed from identical resonators [9, 71-73]. This suggestion was in part based on the results of studying the resonances in single dielectric sphere, i.e. so-called Mie resonances named after one of the first researchers in the field of wave scattering from dielectric particles $[67,74]$. Spheres were found capable of supporting both resonances electric and magnetic, however, these resonances were observed at significantly different frequencies. Some earlier researchers have suggested that for obtaining the left-handed effects coincidence of two resonance frequencies is not necessary and it is enough, instead, to get overlapping of the resonance tails [75-83]. In favor of such suggestion, they presented numerous cases of correlation between overlapping of resonances and observing the negative refraction. However, no detailed studies of the hybrid resonance modes expected at overlapping the resonances were presented and no investigations of alternative reasons for observing negative refraction were conducted. In Chapter 3 we described our studies directed on understanding the origin of negative refraction in all dielectric MMs composed of identical resonators. We reproduced conditions of several works, and did not find, in obtained data, any evidences of either overlapping two resonance modes or double negativity of effective parameters. Our data have shown that negative refraction in MMs composed from identical dielectric resonators is defined by their dispersive properties.

\subsection{Directional wave scattering from metasurfaces of dielectric nano- disk resonators}

Recently, attention of many researchers working in the field of nano-photonics has turned to investigating an opportunity of obtaining directional scattering of light from arrays of dielectric nanoparticles [84-86]. As in the case with negative refraction in adMMs, this phenomenon was from the start considered as resulting from interplay between two dipolar resonances, magnetic (MR) and electric (ER) ones. Such consideration was based on earlier theoretical works on scattering conducted by the Kerker's team [87, 88] who used as the objects under study magneto-dielectric particles, in particular, spheres. This team, in fact, 
continued studying Mie resonances and found that overlapping of the tails of dipolar Mie resonances in particles, at frequencies below the resonance frequency of the first in the spectrum magnetic Mie resonance, could lead to suppression of resonant back scattering (BS), thus making particles supporting only front scattering (FS). This phenomenon was considered as a sequence of occurrence of the so-called $1^{\text {st }}$ Kerker's condition, at which waves scattered by spheres at electric and magnetic resonances obtained phase difference providing for their destructive interference. The results of Kerker's team works were later confirmed for dielectric spheres operating in microwave [89] and optical (in the case of nano-dimensions) [14] ranges. It was noticed in [90], however, that directional FS from ordinary dielectric spheres cannot be enough strong for practical applications in photonics. In order to enhance scattering, it seemed worthwhile to find the opportunities for shifting magnetic (MR) and electric (ER) resonances closer to each other in the frequency spectrum. It appeared that spherical $[14,89]$ and pillar-type [91] dielectric particles could not be used for such purpose, since they had only one dimensional parameter for controlling the resonant frequencies. Therefore, there appeared studies of scattering from particles with other than spherical shapes, in particular, of spheroids [92] or hollow cylinders [93, 94]. However, these geometries were not practical for using in optical range. Therefore, disklike particles, possessing two parameters (thickness and diameter) for controlling the frequencies of MRs and ERs, have been considered for applications in photonics in [15, 95]. It was shown that at the aspect ratio of about 1:2 [15], frequencies of two resonances in such disks could be shifted up to coincidence. In the case of coincidence, the $1^{\text {st }}$ Kerker's condition had to be achieved, supposedly, at the common resonance frequency, thus providing powerful FS at zero backward scattering (BS). However, it is worth mentioning here that resonance modes in disks were expected to have few in common with modes of Mie resonances in spheres and that it demanded justification of extending the Kerker's concepts to disk-like particles. Despite it was not done so far, researchers moved to next steps suggesting the possibility of extending the Kerker's concepts to planar arrays of dielectric nano-disks (metasurfaces) [15, 95].

The data presented for metasurfaces of silicon nano-disks in [15, 95], caused some questions. In particular, supposed coincidence of two resonances in [15, 95] was 
accompanied by full transmission through the arrays in relatively wide frequency range, while no signs of resonance occurrence were observed. In addition, authors of [95] advertised $2 \pi$ phase shift in FS with respect to incident wave (i.e. no phase shift, in fact). Expectations of such shift were based on suggestion that two phase shifts by $\pi$ in oscillations of electric and magnetic dipoles could be combined. However, this suggestion omits from consideration the fact that shifts of the phase at resonances occur with respect to the phase of incident wave and not with respect to each other.

Described above extension of Kerker's concepts to arrays of nano-disks pushed us to conduct more careful investigation of responses from nano-disk based DR arrays without considering them as a kind of metamaterials with effective parameters. In addition, taking into account the results of described in Chapter 3 studies of adMMs we did not exclude the possibility to reveal in metasurfaces phenomena defined by dispersive properties of these periodic structures. Therefore, we also compared and analyzed responses of disk arrays at different lattice constants.

\subsection{All-dielectric photonic crystals for transformation optics based devices}

The year 2006 was marked by publication of seed papers introducing new theory called Transformation Optics (TO), which provided an approach for developing new electromagnetic devices capable of changing the paths of waves in chosen media in any desired way $[17,96]$. The TO was based on the form-invariance of Maxwell equations in different coordinate systems, which allowed to control wave paths using coordinate transformations of the original space into a new arbitrary space. The equivalence of the

original and transformed space for the wave propagation could be accomplished by setting prescribed anisotropic permittivity and permeability tensors for the transformed space following specific mapping functions. Figure 1.4 shows the field trajectory in both the original and an arbitrarily transformed space. 


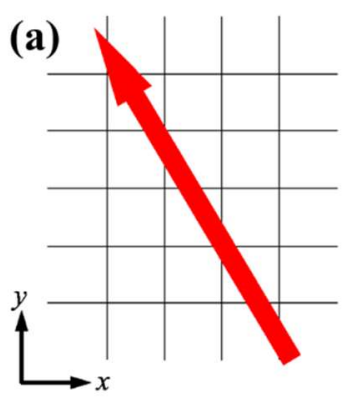

Original

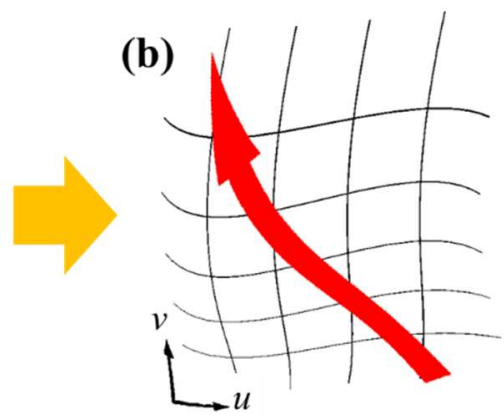

Transformed

Figure 1.4 (a) field line in free space in the original medium, (b) the distorted field line in transformed medium

The TO has led to creation of an invisibility cloak i.e. the device which could hide any object at placing it inside respective shell. When electromagnetic waves entered the cloak medium, they were guided around the cloaked object and then followed along the original trajectory, as if the object did not exist. (Fig.1.5) This intriguing device has been first implemented for operation at microwave frequency [96]. It used as the cloak medium providing requested parameters for coordinate transformation the MM composed from split-ring resonators. However, employing such conventional MMs excluded a possibility to extend the frequency range of the invisibility to higher frequencies even in mm-wave range, nothing to tell about optics. The problem was in exponentially increasing with frequency losses controlled by the skin effect in metallic resonators.

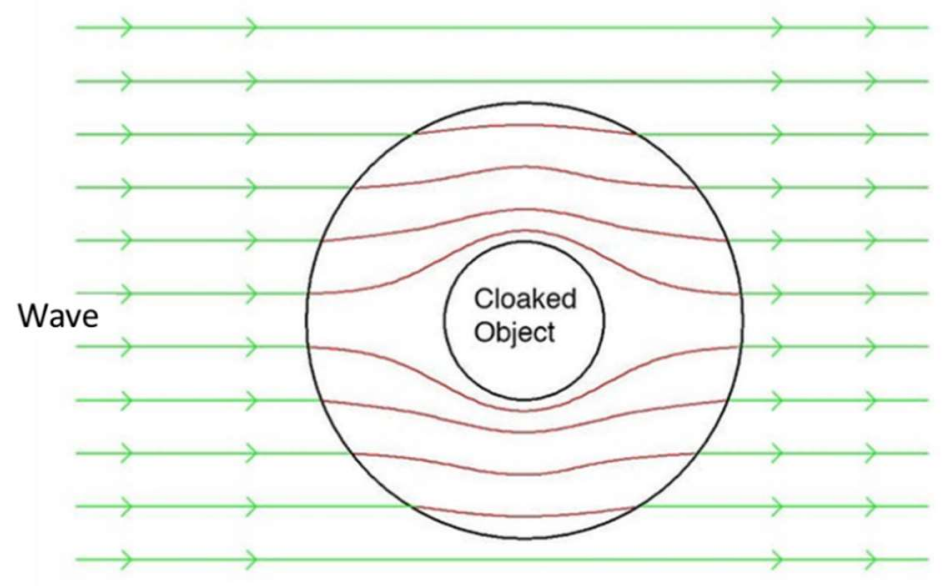

Figure 1.5 An invisibility cloak surrounding the object changing the wave trajectory

TO-based prescriptions for the cloaking shell demanded the medium relative effective permittivity or permeability to have values between zero to one to support the superluminal 
phase velocities around the object. Superluminal phase velocity provided accelerating the waves moving along curvilinear paths around the object thus allowing for the restoration of original flat wave front beyond the cloak. Since regular materials could not provide the above-mentioned values of permittivity and permeability $(0<\varepsilon, \mu<1)$, metamaterials (MMs) seemed to be good candidates for designing the cloak $[96,97]$. The effective permittivity at electric resonance and permeability at magnetic resonance of MMs were expected to approach zero at plasma frequency, $f_{p}$, and cause superluminal phase velocities of propagating waves. The cloak in [96] was designed from concentric layers, each of them was hosting split-ring resonators (SRRs) with dimensions providing for slightly different values of $f_{p}$ to realize gradually changing superluminal responses from layer to layer. The resonance nature of MMs and described design specifics of the cloak made cloaking effect extremely narrowband. In addition, an employment of metal SRRs in the cloak led to delayloss constraints, which restricted the maximum size of cloaked object by a few wavelengths of the incident wave [98]. Finally, the saturation of magnetic response could additionally complicate scaling the cloak to THz and optical ranges [99].

Employing metamaterials comprised of dielectric resonators (DRs) instead of split-ring resonators was expected to provide much lower loss and better scalability [73, 100]. An all-dielectric cloak for $\mathrm{THz}$ range was firstly proposed in 2008. However, its design assumed employing DRs of different sizes that was impractical. Better cloak design suggesting employment of identical DRs all over the cloak was proposed in [73]. This spoke-looking cloak employed air fraction for realizing TO requested spatial dispersion of effective permeability and was shown as capable to perform in the infrared range. It was found that it could conceal metal cylinders with diameters of 5 times greater than wavelengths of incident radiation. However, due to the resonance nature of metamaterial structures, the cloak conserved such drawback as the narrowbandness with the bandwidth of cloaking effect not exceeding $1.2 \%$.

In [101] it was assumed that better cloaking performance could be expected at employing in the cloak medium all-dielectric photonic crystals, instead of MMs. In [101, 102] it was also shown that cloak media formed by $1 \mathrm{D}$ PhCs can bend wave paths by similar way as an SRR medium does. In later work of our team [103] we have shown the possibility to 
realize the effect of invisibility of metallic cylinder at using cylindrical cloak with medium formed of dielectric rods. In this dissertation in Chapter 5, we highlight some problems, solving of which is important for implementing the PhCs in transformation media and describe some of efforts undertaken to solve these problems.

\subsection{Objectives}

The objective of this dissertation is to advance functionalities of selected EM devices by proper using their dielectric-based media and to deepen an understanding of the processes, which control electromagnetic responses of the media, in particular, their refractive properties, electric and magnetic resonances in their constituents, and their performance in transformation optics based devices.

The target of the first part of our work, presented in Chapter 2, was developing novel RF probes for ultra-high field MRI scanners. The idea used for this purpose suggested employing antenna-inspired patch structures as resonators capable of supporting relatively strong magnetic field outside the resonance cavity (above the patch electrode). Our goal was to propose and test various approaches to designing both surface and volume RF probes for UHF scanners. One of the approaches was based on replacing the uniform substrate in patch structures by multi-dielectric substrate engineered to affect the uniformity and strength of magnetic field above the patch electrode. Another approach suggested employing substrates of higher permittivity and using patches with modified geometry, i.e. patches with profiled shapes. To verify the results of simulations, we intended to test the prototypes of novel RF probes in 14.1 T MRI scanners. In addition, the performance of the novel probes had to be compared with the performance of conventional probes utilizing birdcage coils.

The purpose of work presented in Chapter 3 was to clarify the physics of processes defining the phenomenon of negative refraction observed in the media formed by dielectric resonators. Understanding of refraction phenomena in DR arrays was critical for both adequate description of DR arrays as physical objects and application of DR arrays for controlling EM waves. Most of published literature described DR arrays as metamaterials and related the negative refraction of light in arrays to overlapping of electric and magnetic 
resonances in dielectric particles that was supposed to lead to double negativity of effective parameters. No works directly checked the role of array dispersion properties in observed phenomena. This situation allowed to formulate the first task of our investigation as studying the dispersion diagrams of DR arrays, which demonstrate negative refraction. In addition, we have planned to check how this phenomenon depended on array lattice parameters and to compare transformations produced by changing the array lattice parameters in dispersion diagrams and in the spectra of scattering coefficients. Building up equi-frequency contours, simulations of field patterns, calculations of Mie resonance spectra, and numerical experiments, aimed at distinguishing between forward and backward wave propagation, have been also incorporated in our plans.

The work presented in Chapter 4 aims to provide deeper insight into the physics of phenomena defining the so-called unidirectional scattering from metasurfaces composed of dielectric resonators. This term is used for planar arrays of dielectric resonators, which have been considered by many researchers as a kind of planar metamaterials. As in the case with negative refraction, the dispersive properties of these arrays were largely ignored, so that they were treated as homogenized media composed of identically responding particles, while array periodicity was considered as insignificant for their responses. Thus, the analysis of scattering from metasurfaces was built on employing the concepts developed 35 years ago for single spheres at Mie resonances in them. At that time, it was shown that overlapping of the tails of electric and magnetic Mie resonances should lead to destructive interference in the backward scattered light flow and, thus, to unidirectional front scattering, which was related to approaching the so-called Kerker's conditions. Suggested possibility of similar phenomena in metasurfaces of nano-disks caused booming interest in photonics. However, extension of Kerker's concepts to such metasurfaces was not justified properly so far. Moreover, taking into account the results of described in Chapter 3 studies of adMMs, we could not exclude the possibility to reveal in metasurfaces the phenomena defined by dispersive properties of these periodic structures. Therefore, as the first task we have chosen an investigation of the effects of lattice constants on scattering from metasurfaces composed from nano-disks. Our further plans included control of expected spectral 
changes for electric and magnetic resonances and estimates of correlation between scattering spectra and the specifics of dispersion diagrams for stacked metasurfaces.

The goals of Chapter 5 were solving the problems accompanying incorporation of alldielectric photonic crystals in the transformation media of invisibility cloaks. The first goal of our studies, defined by the analysis of approaches used at designing the first cylindrical invisibility cloak on photonic crystals, was to obtain the data providing reasonable choice of photonic crystals, which have to support wave propagation with superluminal phase velocity in the cloak medium. The second goal was defined by the fact that used in the cloak design fragments of photonic crystals were presented by sets of circular arrays with close lattice constants. It was necessary to define, what quantity of such arrays should be used in each fragment so that its responses corresponded to the dispersion diagrams calculated for infinite arrays. Without answering this question, it was impossible to realize requested by TO radial dispersion of index values at designing the cloak. In addition, we had to clarify how neighboring arrays of $\mathrm{PhC}$ fragments could interact in the cloak affecting the pattern of wave propagation. Finally, the mentioned above analysis has shown that the performance of the first invisibility cloak based on PhCs did not correspond to the case of radial dispersion of only azimuthal index components, which was provided by the cloak design. Field patterns of wave propagation in the cloak cross-section pointed out at bending wave paths inside the cloak, which should have occurred only at realizing radial dispersion of radial index component, not provided by the cloak design. Analysis of the wave patterns let us to assume that the revealed discrepancy was caused by involvement of the selfcollimation phenomena. It defined the new goal - to obtain additional data about selfcollimation phenomena in bent PhCs. 


\section{Developing and Experimental Testing of alternative RF probes for 14T MRI Scanners}

\subsection{Introduction}

Pursuing the goal to develop RF probes for ultra-high field scanners using patch structures, we decided first to investigate opportunities of employing properly engineered multidielectric substrates for controlling the uniformity and strength of magnetic fields formed above patches at the resonances in these structures. Then we modelled when engineered patch playing the role of a surface coil and compared the performances of this patch and two coils with various diameters. To verify the results of modeling, we used engineered patch as the source of B1 field in MRI scanner and obtained the image of magnetic field above the patch. The obtained results were used for developing volume RF probe by using the system of two vis-à-vis placed patches. Modeling of such probe ensured the possibility of creating a highly uniform magnetic field in the space between two patches due to overlapping fields originated from them. The results of modeling were then confirmed by tests in MRI scanners. Further, we employed profiling of patch structures to increase the volume with high magnetic field uniformity so that bigger part of the bore volume could be used for sample placement. Finally, we have shown by simulations that patch-based probes are capable of providing higher stability of Q-factors at their resonances compared to birdcage-type probes at loading both by identical lossy phantoms. Full-wave simulations in this work have been conducted by using CST Microwave Studio software package. Experimental measurements have been performed in the vertical bore of VARIAN 14.1T MRI scanner.

\subsection{Patch with Engineered Multi-Dielectric Substrate as the Surface Probe}

At the resonance in MPA, oscillating surface currents flow along the length of the patch and their density changes from zero near radiating edges to maximal value in the middle of patch length, while opposite electric charges are distributed from maximal densities near radiating edges down to zero values in the middle of patch length. The length of the patch 
electrode in MPAs is about half-wavelength of the waves oscillating within the substrate cavity, while the width of the patch electrode is typically 1.5 times larger than its length. Patches with conventional for MPAs dimensions, however, could not be employed as RF probes in 14T MRI scanners, since, to fit the bore size, the widths of patches should not be larger than 2-3 centimeters, while the patch lengths, at typical low permittivity substrates, should not be disproportionally big at the wavelengths of about $50 \mathrm{~cm}$. Taking into account the above facts, we considered an opportunity to decrease the lengths of patches by embedding high permittivity dielectric inserts in low permittivity substrates as it was done in [104]. Simultaneously, we employed embedding as a tool for controlling magnetic fields above patch electrodes. In particular, placing inserts at the corners of substrate cavity promised to equalize the distribution of current density along both patch width and length, and thus, to provide higher uniformity of magnetic field above the central part of the patch. (It could be noted here that equalizing of current density along the patch length could be expected due to redistribution of electric charges at embedding inserts with shifting the center of "charge gravity" closer to radiating edges [105]).

The prototypes of microstrip patch-based RF probes were fabricated using FR-4 ( $\varepsilon_{\mathrm{r}}=4.4$, thickness of $1.6 \mathrm{~mm}$ ) as the host substrate material and four cylindrical $\mathrm{SrTiO}_{3}$ pellets $\left(\varepsilon_{r}=300\right)$ as inserts (Figure $2.1 \mathrm{~b}$ ). Pellets were embedded into cavities made in the host material. The diameter of each pellet was equal to $5.6 \mathrm{~mm}$ and the thickness - to $1.6 \mathrm{~mm}$, i.e. similar to the substrate thickness. Patch dimensions were equal to $19 \mathrm{~mm} \times 30 \mathrm{~mm}$, while the substrate dimensions were $30 \mathrm{~mm} \times 40 \mathrm{~mm} \times 1.6 \mathrm{~mm}$ (Figure 2.1a). All dimensions were chosen based on preliminary simulations to provide the patch resonance at $600 \mathrm{MHz}$, i.e. at the operating frequency of 14T MRI scanners. Patch and ground plane electrodes were made of copper tape and attached to the substrate with pellets by using silver paint. The patch probes were fed through the hole in the ground plane by using a semi-rigid coaxial cables and BNC connectors. The location of the feeding point was chosen to provide input impedance of $50 \mathrm{ohms}$. To determine the feed location, we, first, simulated dependencies of patch input impedance on frequency at different locations of the feeding point. As seen from Figure 2.1c, the input impedance of $50 \mathrm{ohms}$ at $600 \mathrm{MHz}$ was expected at the location of the feeding point at the distance of $9 \mathrm{~mm}$ from the center of 
patch electrode. Measurements of scattering parameters of several fabricated prototypes with such location of feed point verified the simulation results and confirmed expected resonances in probes with deep dips down to $-20 \mathrm{~dB}$ and below in the S11 spectra. We noticed that the bandwidths of resonance responses in measured S11 spectra were larger than those in simulations that could be caused by losses in layers of silver paint beneath the electrodes. The latter assumption was based on the fact that simulated and measured S11 spectra of patches prepared by using copper-clad laminate structures, such as PCBs, demonstrated good coincidence.

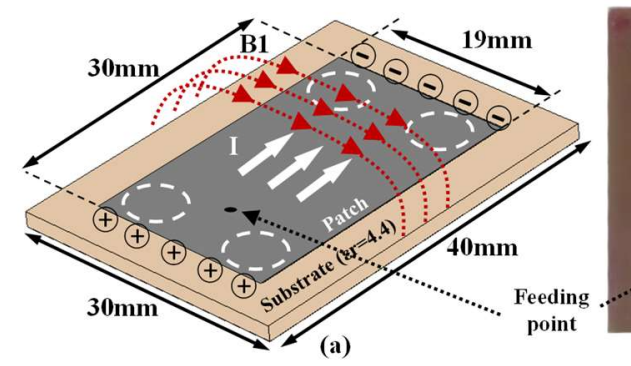

Patch structures

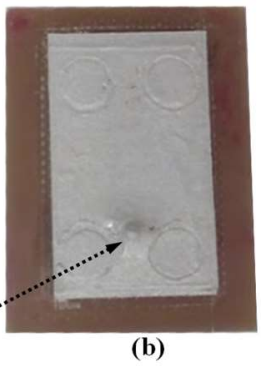

(b)

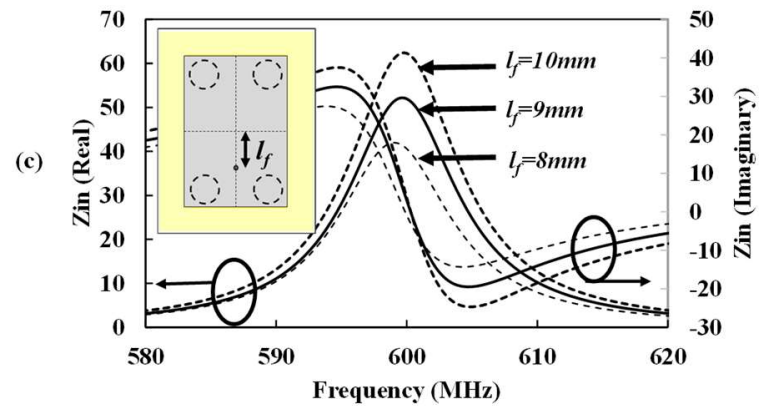

Figure 2.1 (a) Schematic of patch probe showing directions of current and magnetic field: high-permittivity pellets are placed under the patch electrode in the substrate at its corners close to radiating edges; (b) fabricated prototype of the patch probe; and (c) input impedance for different locations of feeding point, ' $\boldsymbol{l}_{\boldsymbol{f}}$ ' shows the distance of feeding point from the patch center.

To judge about uniformity of B1 magnetic fields created above the patch, we, first, present in Figure 2.2a simulated distributions of current density in patch electrodes at the resonance. Three curves in this figure illustrate changes of the current density component $\mathrm{Jz}$, along the patch length at X-coordinates corresponding, respectively, to the middle of the patch width (at $X=0$ ) and to the positions of insert centers near non-radiating edges (at the distance of about $7 \mathrm{~mm}$ from Z-axis of patch symmetry). As seen in the figure, the middle 
portions of all three curves display relatively flat shapes, but although the central curve still has features of cosine function, typical for MPAs, the side curves demonstrate maxima of currents near locations of inserts. Observed shapes of the curves correspond to the expectations of inserts influence on the distribution of current density in the patches. Figure $2.2 \mathrm{~b}$ presents three curves illustrating distributions of transverse magnetic field intensity along $\mathrm{X}$-direction at different $\mathrm{z}$-coordinates, where $\mathrm{Z}=0$ is taken at the middle of patch length. All distributions in Figure 2.2b were obtained in XZ-plane located at the distance of $8 \mathrm{~mm}$ from the patch surface. As seen in Figure 2.2b, all three curves look qualitatively similar to each other that confirms expected positive effect of corner inserts on the uniformity of magnetic field above the patch, despite some decrease of field intensity closer to radiation edges. In addition, Figure 2.3a shows three simulated distributions of magnetic field intensity in the middle of patch length along X-directions taken in XZ-planes located at various distances from the surface of patch. As seen in the figure, the best field uniformity can be seen at the optimal distance of $8 \mathrm{~mm}$ from the patch.

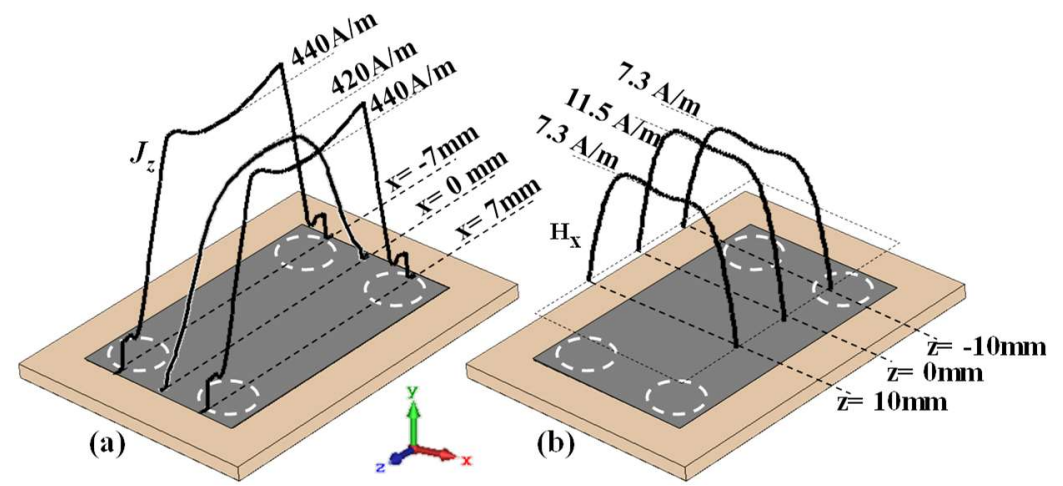

Figure 2.2 (a) Distributions of surface current density $\mathrm{J}_{\mathrm{Z}}$ along $\mathrm{Z}$-axis at different locations along $\mathrm{X}$-axis, where $\mathrm{X}=0$ is taken at the middle of patch width, and (b) distributions of transverse magnetic field intensity $\mathrm{H}_{\mathrm{X}}$ in XZ-plane located at the distance of $8 \mathrm{~mm}$ above the patch along $\mathrm{X}$-axis at different locations along $\mathrm{Z}$ axis, where $\mathrm{Z}=0$ is taken at the middle of patch length.

For comparison, Figures $2.3 \mathrm{~b}$ and $2.3 \mathrm{c}$ show similar changes of field distributions in dependence on the distance from current containing plane, which have to be expected for surface coil probes with two different diameters (in these cases the distributions were simulated along lines parallel to the diameters of coils). As seen in the figures, the coil with the diameter of $19 \mathrm{~mm}$ demonstrated best uniformity of field distribution at the distance of $5 \mathrm{~mm}$ from the coil plane, while often used in practice coil with the diameter of $8 \mathrm{~mm}$ 
provided best result at the distance of $2 \mathrm{~mm}$ from the coil plane. It is worth noticing here that RF probes presented by patches with engineering substrates provide B1 field parallel to the patch surface, while in the case of coil probes, B1 field appears directed normally to the coil plane that can involve in imaging the tissue layers located below the coil plane.

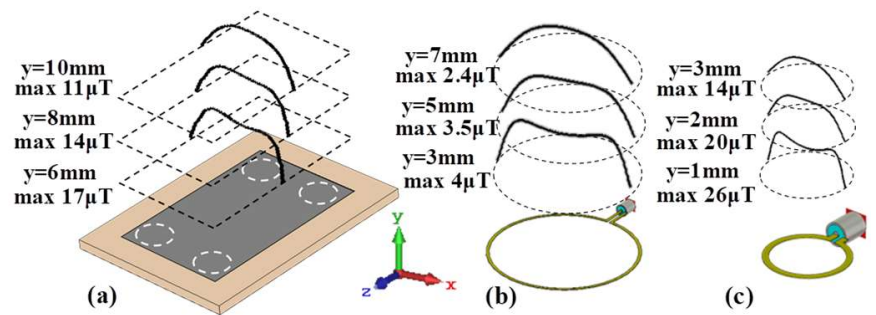

Figure 2.3 Magnetic field distributions along $\mathrm{X}$-axis in $\mathrm{XZ}$ planes located at various distances from currents containing planes of three RF probes: (a) patch with engineered substrate, (b) surface coil with the diameter of $19 \mathrm{~mm}$, and (c) surface coil with the diameter of $8 \mathrm{~mm}$

Figures 2.4a to $2.4 \mathrm{c}$ present snapshots of B1 fields in XZ-planes located at optimal distances from planes, containing currents, in three RF probes presented in Figure 2.3. As seen in the figures, maximal fields were observed above the coil with the diameter of $8 \mathrm{~mm}$, while the intensity of field above the coil with the diameter of $19 \mathrm{~mm}$ was about 6 times less. Fields observed above the patch with engineered substrate were also less than in the case of small coil, however, they were only 1.5 times less, while the area of uniform field looked much bigger. Figures $2.4 \mathrm{~d}$ and $2.4 \mathrm{e}$ compare changes of B1 field intensity along Xand Z-axes in XZ-planes, located at optimal distances from planes, containing currents, in three probes. Since the data presented in Figures $2.4 \mathrm{~d}$ and $2.4 \mathrm{e}$ are normalized, they can be used for estimating the dimensions of areas, in which field intensities for each of three probes remained on the levels exceeding $90 \%$ of the maximal field values. Following [106], such areas can be considered as areas with uniform fields. 


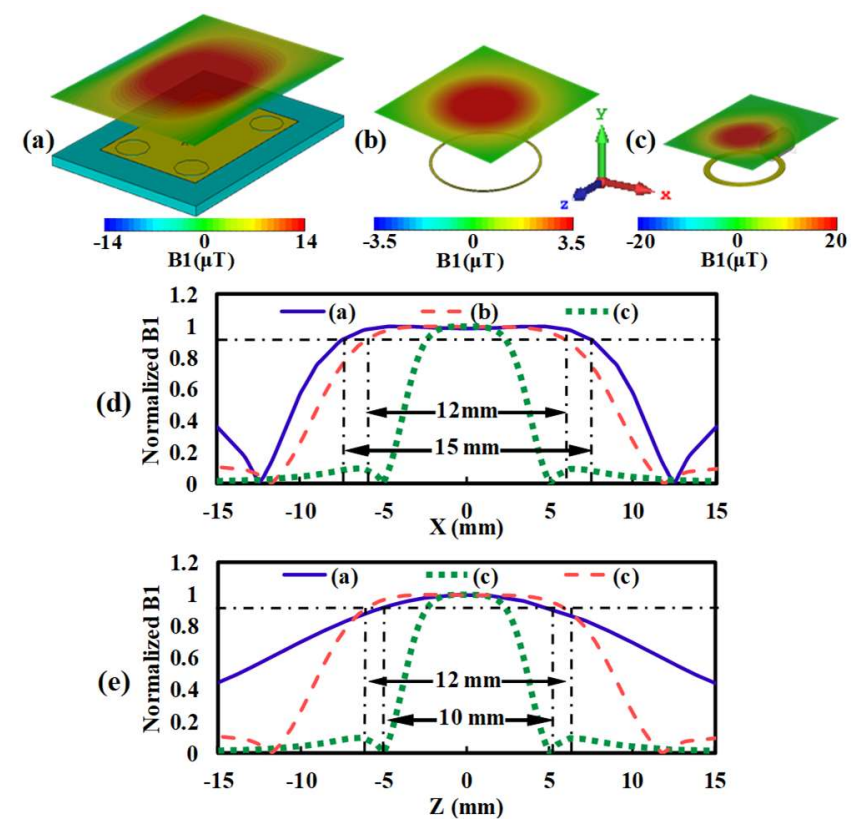

Figure 2.4 (a) - (c): snapshots of magnetic fields distributions in XZ-planes taken at optimal distances from current containing planes of three RF probes presented in Figure. 2.3. (d, e) Spatial changes of B1 field intensity along X-and Z-axes in above mentioned XZ-planes.

In the case of small coil ( $8 \mathrm{~mm}$ in diameter), B1 field intensity remained above $90 \%$ of the maximal field intensity within the circle with the diameter of $4 \mathrm{~mm}$ (green dotted curves in Figures $2.4 \mathrm{~d}-\mathrm{e})$. For the coil with the diameter of $19 \mathrm{~mm}$, i.e. equal to the width of patch with the engineered substrate, the area with similar field uniformity was represented by the circle with the diameter of $12 \mathrm{~mm}$ (red dashed curves in Figures $2.4 \mathrm{~d}$ and $2.4 \mathrm{e}$ ). In the case of the patch with engineered substrate, blue curves in Figures $2.4 \mathrm{~d}$ and $2.4 \mathrm{e}$ allowed to determine X-dimension of the area with uniform field to be of $15 \mathrm{~mm}$, and its Z-dimension to be of $10 \mathrm{~mm}$. Thus, the area of uniform field above the patch with engineered substrate appeared to be almost 1.5 times bigger than that above the larger coil, while field intensity above the patch was 4 times higher than that above the coil. This result makes the patch with engineered substrate to be an advantageous candidate over the large coils for serving as a RF surface probe.

To ensure that placing the patch probe in the bore would not deteriorate predicted by simulations formation of magnetic field above the patch electrode, the patch probe with engineered substrate was tested in the MRI environment. To obtain an image of magnetic field provided by the patch probe, we used special holder for the patch and plastic container 
(usually employed at imaging liquid samples), which was inserted in the vertical bore of 14.1T MRI scanner (Figure 2.5a). This container was filled with the 5\% water solution of MRI contrast substance Magnevist for obtaining a brighter image. It can be seen from comparison of Figures $2.5 \mathrm{~b}$ and $2.5 \mathrm{c}$ that the developed patch probe produces the same type of field inside the bore as that observed in simulations (Figures 2.5b and 2.5c). Obtained results demonstrate that MPA-inspired patch probes can be considered as perspective sources of B1 field at MRI studies.

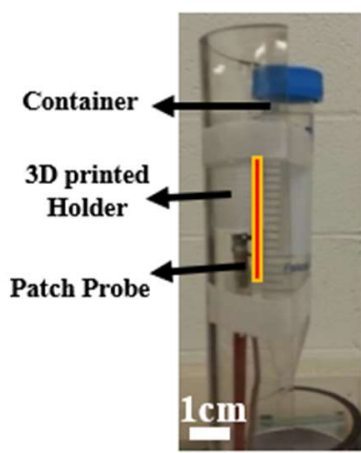

(a)

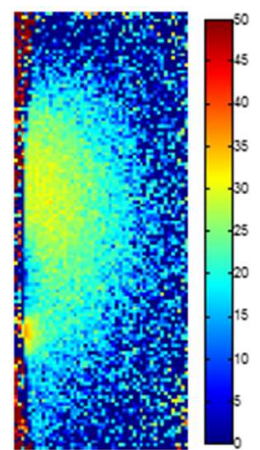

(b)

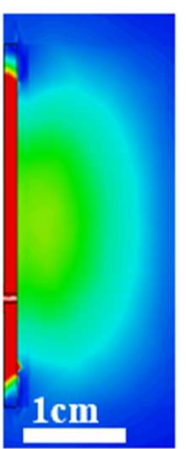

(c)

Figure 2.5 (a) Setup of the patch probe with attached container prepared for insertion in the vertical bore of Varian 14.1 T MRI system, (b) MRI image of B1 magnetic field within container, and (c) snapshot of simulated magnetic field distribution above the patch probe.

An important problem that had to be addressed at MRI applications of proposed patch probes was the problem of tuning the resonance response of patches at their placing inside the bore and at loading them by tissue samples prepared for imaging. We employed literature devoted to the analysis of MPAs $[107,108]$ to choose an equivalent circuit for modeling patch probes and then considered the effects from incorporating tunable reactive elements in this circuit (Figure 2.6a). $X_{L}$ in Figure 2.6a takes into account the inductance of the coaxial feed line. Tunable elements $\left(C_{t}\right)$ should be used to shift the resonance frequency of the probe back to $600 \mathrm{MHz}$ after its increase due to effects of the bore and inserted samples. It was found that this function can be provided by tunable capacitors connecting patch electrodes and ground planes.

Figure $2.6 \mathrm{~b}$ shows how the value of incorporated capacitance affects the resonant frequency. We see that by employing $5 \mathrm{pF}$ capacitor (such as Knowles-Voltronics AP14 trimmer, which is variable between $1 \mathrm{pF}$ to $14 \mathrm{pF}$ ), the resonant frequency can be shifted down by 
about $12 \mathrm{MHz}$, which was found sufficient for mitigating the effects of possible frequency shifts.
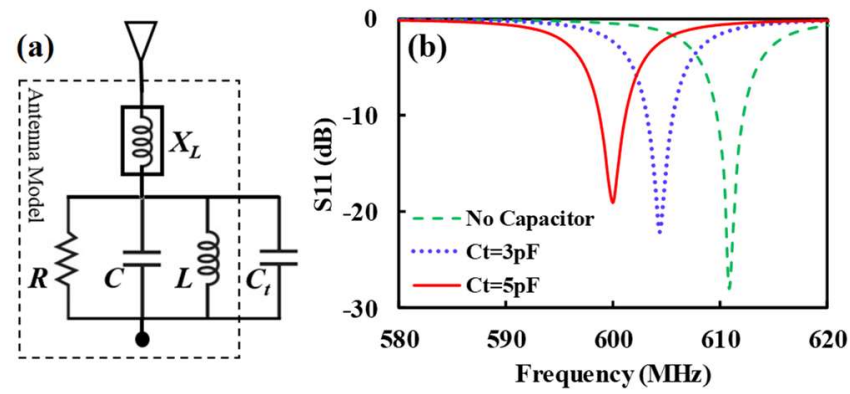

Figure 2.6 (a) equivalent circuit of the coax-fed antenna with the tuning capacitor; and (b) the effect of tuning capacitor introduced between patch and ground plane electrodess on restoring resonance response of the patch back to $600 \mathrm{MHz}$ after its shifting at operating with samples.

Conducted studies allow for recommending patch RF probes to be used as surface probes in MRI systems.

\subsection{System of vis-à-vis Placed Patches as Volume Probe}

Verified in the previous section opportunity to use patch probes for the formation of uniform and strong magnetic field above patch surface points out at a possibility to use two similar patch structures, placed vis-à-vis in the bore with patch electrodes facing each other, for obtaining uniform B1 field in some volume between patches. Such system of two patches could be employed similar to volume coils for studies of 3D objects. To provide additivity of magnetic fields formed by two vis-à-vis placed patches, they should be fed with $180^{\circ}$ phase difference. For this purpose, both patches could be fed from one source, but one of two feeding lines connecting the source with patches, should have a halfwavelength transformer, as it shown in Figure 2.7a (transformer was designed considering the effective wavelength in RG58 coaxial cables at $600 \mathrm{MHz}$ ).

It should be noted here that at the proposed schematic of feeding, two electric fields formed along patch lengths, which control current flow in two patches, should be directed oppositely and, thus, should compensate each other in the 3D volume of their overlapping between patches. Therefore, these fields should not affect samples placed in the inter-patch space. This is important, since electric fields penetrating in conductive tissues of samples under study could induce electric currents in them that can cause tissue heating, capable of 
damaging the samples [109]. However, there is a concern that additional electric fields could be built between vis-à-vis located edges of two patches, since these edges appear oppositely charged. To avoid such negative effect, the distance between two patches should be optimized to be not too small, but still suitable for efficient overlapping the magnetic fields originating from two patches. For experimental testing of formed by two patches volume probe in the bore of 14.1T MRI system, we fabricated a special holder shown in Figure 2.7b.
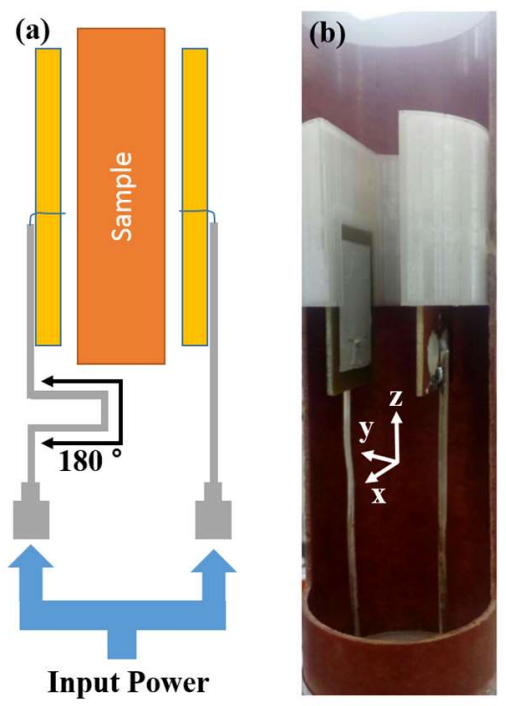

Figure 2.7 (a) Schematic of the system of two patches facing each other and (b) fabricated setup with two patches well distant from each other.

In experiments with new volume probe, the phantom of tissue sample was represented by homogenous mineral oil placed in cylindrical container located between two patches. First, the images of the phantom in XY-cross-section (see Figure 2.7b) were taken at turning on only one or another of two patches, while the counterpart was disconnected from the source (Figures 2.8a and 2.8b). 

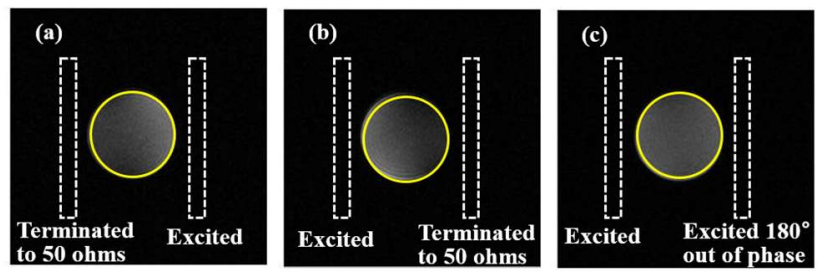

Figure 2.8 Transverse cross-sectional images of the liquid sample by using the volume probe composed of two patches with engineerd substrates: (a) at feeding only the patch located in the figure to the right of the sample, (b) at feeding only the patch located in the figure to the left of the sample, and (c) at feeding both patches with $180^{\circ}$ phase difference. Locations of patches are shown by white dashed contours.

To avoid distortions in source operation at such tests, "disconnected" coax connector was terminated by $50 \mathrm{ohms}$ load. The purpose of these tests was to ensure counter-symmetry of the obtained images. Then, to ensure additivity of magnetic fields from two patches at their operation inside the bore, the image of the phantom was obtained at turning on both patches (Figure 2.8c). As seen in Figures 2.8a and 2.8b, employing only one of two patches, constituting the volume probe, led to appearance of a contrast between two halves of phantom images, i.e. that half of the image, which was located closer to the active (turned on) patch looked bright, while the opposite half was darker. When two patches were fed with the phase difference of $180^{\circ}$, the image of B1 field formed in the phantom by two patches (Figure 2.8c) looked as the result of overlapping images given in Figures 2.8a and $2.8 \mathrm{~b}$, and appeared well homogeneous.

Figures $2.9 \mathrm{a}$ to $2.9 \mathrm{c}$ demonstrate images of the phantom in three orthogonal cross-sections of the volume probe (images were obtained at turning on both patches). As seen in the figures, images obtained at crossing the phantom by XZ- and YZ-planes also confirm high uniformity of B1 field in the central part of the inter-patch space. To obtain quantitative estimates of the field uniformity and to determine the size of the volume with field intensity exceeding $90 \%$ of the maximal value, we extracted the data characterizing B1 fields in phantoms from MRI test results. To realize this procedure, we used the software from the control panel of VARIAN 14.1T MRI scanner. 

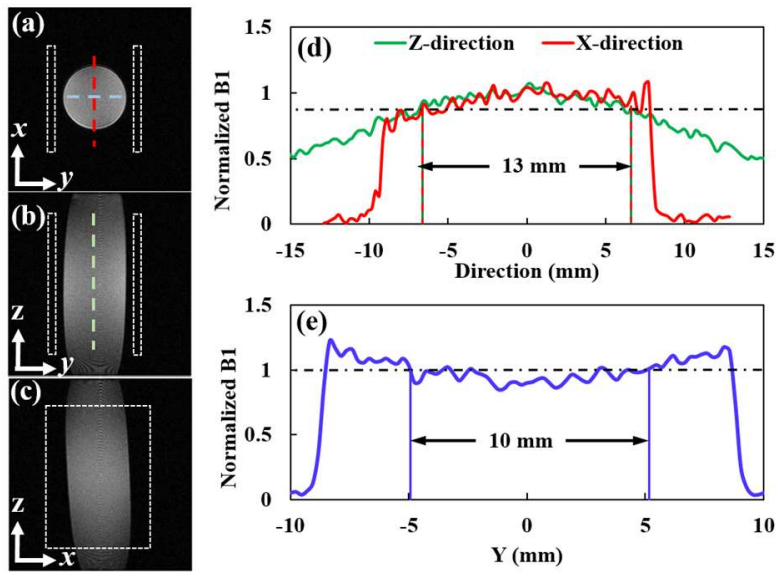

Figure 2.9 (a)-(c) Images of the phantom at three orthogonal cross-sectional planes: (a) XY, (b) YZ, and (c) $\mathrm{XZ}$, and (d)-(e) distributions of B1 field intensity along three axes of phantom symmetry within above listed cross-sections. Locations of patches are shown schematically by white dashed contours.

Figure $2.9 \mathrm{~d}$ presents the results of extraction of B1 field values normalized with respect to their maximal levels in the center of probe symmetry. The data in Figure 2.9e, in which the curve has minimum in the center of probe symmetry, were normalized with respect to a value $B_{N}$, which was found from considering the minimum value of $B 1$ field intensity in the center of probe to be equal to $0.9 \mathrm{~B}_{\mathrm{N}}$. Thus, in this case, we normalized the data with respect to the value of field intensity at some distance of about $6 \mathrm{~mm}$ from the center, and not with respect to values of maximal B1 field intensity observed near the edges of the graph. It is worth mentioning here that the drops of B1 values down to zero, seen in Figures $2.9 \mathrm{~d}$ and $2.9 \mathrm{e}$, are not related to real configuration of fields produced by patches and are defined, instead, by the borders of the phantom body. Presented in Figures 2.9d and 2.9e changes of B1 field intensity along coordinate axes could be compared to simulation results presented for a single patch probe in Figure 2.4. In particular, it is possible to see that the curves showing changes of B1 field intensity along Z-direction in Figures 2.4e and 2.9d are quite similar. Then, while simulated changes of B1 field intensity along X-direction above the single patch in Figure 2.4d demonstrate the length of field uniformity area of about $15 \mathrm{~mm}$, experimental data in Figure 2.9e allow for determining the length of similar area as equal to $13 \mathrm{~mm}$. The lengths of field uniformity areas along Z-direction in two cases are also close, i.e. $12 \mathrm{~mm}$ and $10 \mathrm{~mm}$. Thus, a good correspondence between the simulated and experimental data is observed. It is worth noting in addition that the data presented in 
Figure 2.9 confirm the formation of a 3D volume with uniform fields around the center of probe symmetry. The size of the volume with B1 field intensity exceeding 0.9 of its maximal value could be estimated as equal to $13 \mathrm{~mm}$ in both $\mathrm{X}$ - and Z- directions and to $10 \mathrm{~mm}$ in Y-direction. These numbers characterize the cuboid-type volume, which could be used for placing a sample inside the volume RF probe.

\subsection{Systems of Profiled Patches as Volume Probes}

As shown in previous sections, patches with engineered multi-dielectric substrates are capable of providing in space acceptable B1 field distributions at the resonance and can be employed as surface probes and volume probes. In this section, we investigate another opportunity for controlling B1 fields by modifying patch shape, i.e. by profiling patch structures with uniform substrates (Figures 2.11a and 2.11c). In order to miniaturize these patches, we had to employ substrates with higher permittivity. However, to decrease dimensions of patches with uniform substrates down to dimensions of patches with engineered substrates, we would have to use dielectrics with permittivity of about 80 that could cause problems with impedance matching. Therefore, we selected $1.6 \mathrm{~mm}$ thick flexible Rogers 3010 substrates $\left(\varepsilon_{\mathrm{r}}=10.2\right.$ and $\left.\tan \delta=0.001\right)$, which could be formed in cylindrical and even more complicated shapes. To obtain the resonance at $600 \mathrm{MHz}$ in the patches with such substrates, the lengths of their patch electrodes had to be increased up to $80 \mathrm{~mm}$, while their widths were restricted by the diameter of the bore and, therefore, for the flat substrate, the width was close to the widths of patches with engineered substrates (22 $\mathrm{mm}$ over $19 \mathrm{~mm}$ ). For cylindrical and cosine-profiled substrates, the widths of patch electrodes were kept close to the patch electrode width in the case of flat substrate, i.e. they did not exceed $26 \mathrm{~mm}$. Dimensions of substrates were also close to those in flat substrate case - of about $30 \mathrm{~mm} \times 100 \mathrm{~mm}$. Although dimensions of such patch structures were very different from dimensions of typical MPAs for $600 \mathrm{MHz}$, resonance processes in devices

of both types were expected to be basically similar. Large lengths of patches along the axis of bore did not create problems for imaging samples of small sizes, while were convenient for investigating elongated samples. For matching the input impedance of the probe to 50 ohms, we again conducted described in section 2.2 parametric studies to find optimal 
position of the feeding point. As it is shown in Figure 2.10, input impedance of $50 \mathrm{ohms}$ at the patch resonance could be obtained, if feeding point is located on Z-axis of patch symmetry at the distance of $5 \mathrm{~mm}$ from the patch center. It can be noticed from the figure, that real part of input impedance changes by about $20 \mathrm{ohms}$ per each $1 \mathrm{~mm}$ change in the location of the feeding point. By comparing this change of impedance with that for the patch with engineered substrate (around $10 \mathrm{ohms}$ per each $1 \mathrm{~mm}$ ), we see that impedance for patches with substrates of higher permittivity is more sensitive to the location of feeding point.

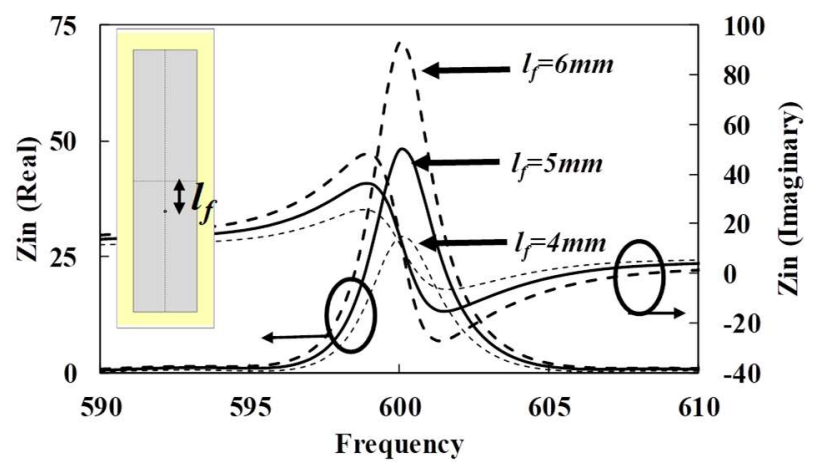

Figure 2.10 Input impedance at different locations of feeding point in the long patch probe, ' $l_{f}$ ' shows the distance between feeding point and the patch center.

Figure 2.11 illustrates differences in magnetic field patterns formed above single patches with various substrate shapes. From the snap-shot of magnetic field in the XY-cross-section of the patch with flat substrate (Figure 2.11a) it is seen that areas with high intensities of the fields above this patch are located near non-radiating edges of patch, while in the central part (dashed circle) the distribution of magnetic field is essentially non-uniform. In the case of patch with cylindrical substrate shape (Figure 2.11b), areas with high intensity of magnetic field appear shifted up with respect to the substrate center that leads to formation of uniform magnetic field in the central part of space above the patch center. Additional profiling of the substrate, i.e. making it "cosine-shaped" (Figure 2.11c), increases the area with uniform field and shifts it a little closer to the patch center.

For building up the volume probes, patch structures of three types were placed vis-à-vis, as shown in Figures 2.11a and 2.11c, and fed with $180^{\circ}$ phase difference (see section 2.3). 
In order to provide comparison of probe functioning, the distances between the centers of two patches were chosen to be of about $30 \mathrm{~mm}$ in all three cases.

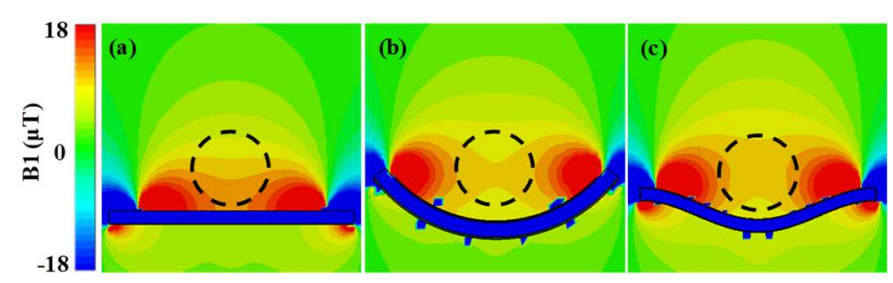

Figure 2.11 Simulated snapshots of distributions of magnetic field intensity in XY-cross-sections of patch probes with various substrate shapes: (a) flat, (b) cylinderical, and (c) cosine-profiled. Dashed circles show the areas convenient for sample placing above patches.

Although patches with cylindrical and cosine-type shapes, i.e. with shapes more conformal to the shape of the bore, were expected to be more efficient at employing the bore space, we concentrated our attention, first of all, on the uniformity of magnetic fields within convenient for placing the samples cylindrical volumes with the diameter of $20 \mathrm{~mm}$ (and arbitrary length up to $80 \mathrm{~mm}$ ) located in the inter-patch space. Cross-sections of these volumes are shown in Figures 2.12a to 2.12c by dashed circles.

As seen from snap-shots of magnetic field distributions in XY-cross-sections of the probe inner spaces (Figures 2.12a to 2.12c), the probe based on patches with flat substrates demonstrated relatively small in size area with uniform field distribution in the central part of inter-patch space, while probes based on patches with profiled substrates demonstrated much larger areas with uniform fields. In particular, the probe with cylindrical shapes of patches produced an extended area of field uniformity along Y-direction, while the probe composed of patches with cosine-type shapes provided uniform fields within almost entire area of dashed circle.

Figures $2.12 \mathrm{~d}$ to $2.12 \mathrm{f}$ compare magnetic field changes along coordinate axes passing through the centers of symmetry in three volume probes. As seen in Figure 2.12d, the lengths of the volume with uniform field along X-axis do not exceed $12 \mathrm{~mm}$ in the probes composed of patches with flat and cylindrical substrates, while in the probe composed of patches with cosine-profiled substrates the respective length reaches $18 \mathrm{~mm}$. 


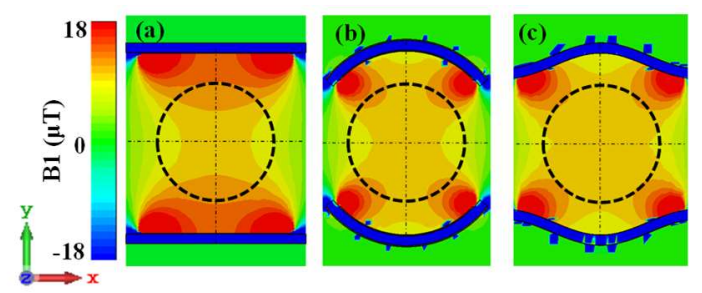

(d)

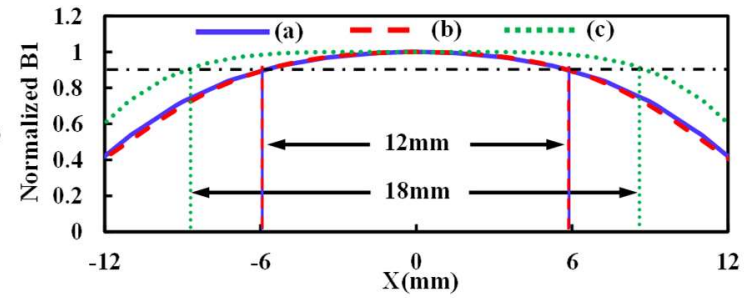

(e)

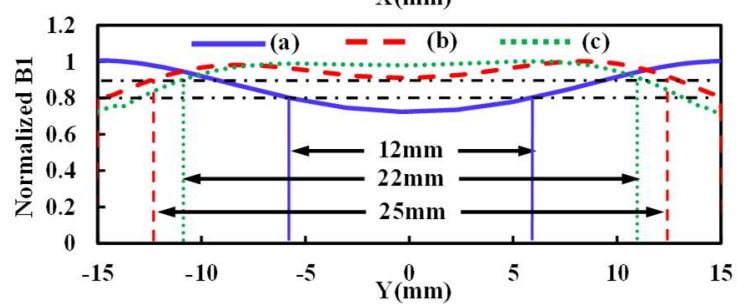

(f)

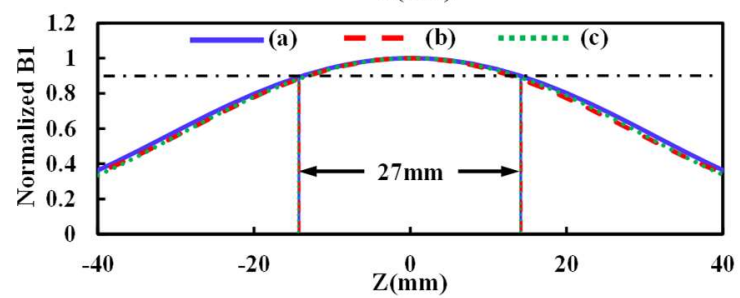

Figure 2.12 (a) - (c) Obtained by simulations snapshots of magnetic field distributions in XY-cross-sections of volume probes with different substrate shapes: (a) flat, (b) cylindrical, and (c) cosine-profiled; dashed circles with diameters of $20 \mathrm{~mm}$ show the areas of interest for placing samples. (d), (e) Characteristic for three volume probes changes of magnetic field intensity along coordinate axes passing through the centers of volume probe symmetry: (d) in X-direction, (e) in Y-direction, and (f) in Z-direction (along the bore axis). $\mathrm{X}$ and $\mathrm{Y}$ axes are shown by dashed dotted lines in (a) - (c).

In Figure $2.12 \mathrm{e}$, the curve characterizing changes of magnetic field intensity along Y-axis for the probe with flat patches has minimum in the center of probe symmetry that agrees with the data presented in Figure 2.12a, while the length of volume with uniform field along Y-axis for this probe is only $12 \mathrm{~mm}$. In contrast, the probes composed of profiled patches demonstrate essentially larger lengths of the volumes with uniform field in Ydirection, exceeding $25 \mathrm{~mm}$ in cylindrical case and $22 \mathrm{~mm}$ at using cosine-profile patches. Along Z- direction (Figure 2.12f), three probes perform similar, i.e. the lengths of the volume with uniform field along this direction appear to be of $27 \mathrm{~mm}$ in all cases. 
Conducted comparison allows for considering volume probes composed of cosine-profiled patches as most advantageous. Therefore, a prototype of such probe was fabricated and tested in 14.1T MRI system (Figure 2.13a). As in the previous tests of volume probes, a cylindrical container filled with mineral oil was used as the phantom. The diameter of the container was $30 \mathrm{~mm}$. Figures $2.13 \mathrm{~b}$ to $2.13 \mathrm{~d}$ present $2 \mathrm{D}$ images of the phantom in XY-, YZ-, and XZ-cross-sections, respectively. These images demonstrate that B1 field is distributed quite uniformly within the phantom.
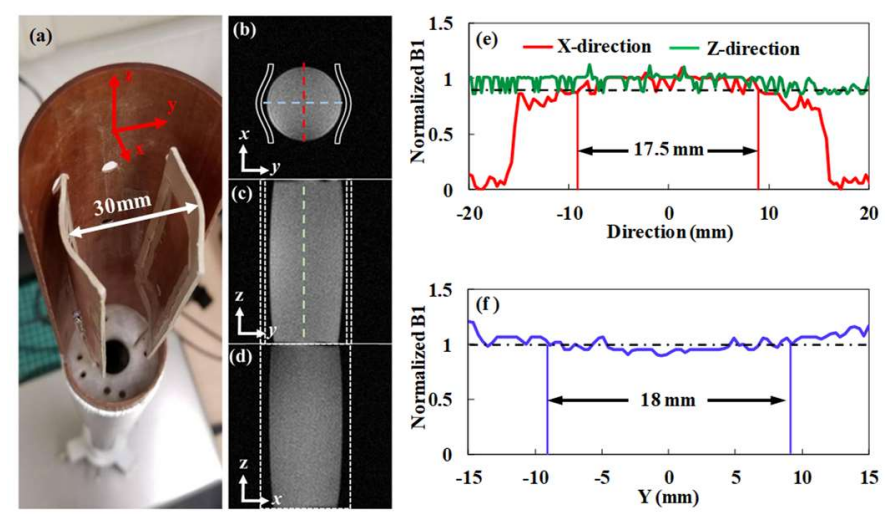

Figure 2.13 (a) Volume probe formed from two cosine-profiled patches is prepared for testing in the bore; (b)-(d) Echo images of B1 field distributions in cross-sections of the sample presented by a plastic container filled with mineral oil; (b) in XY-plane, (c) in YZ-plane, and (d) in XZ- plane. (e) - (f) Extracted at MRI tests graphs presenting changes of B1 field intensity along coordinate axes passing through the center of volume probe.

Obtained at the MRI tests graphs representing changes of B1 field intensity along coordinate axes passing through the center of phantom symmetry are shown in Figures $2.13 \mathrm{e}$ and 2.13f. It can be seen from the figures that the probe with cosine-profiled patches demonstrates the width of volume with uniform B1 field in X-direction of $17.5 \mathrm{~mm}$ and the width of volume with uniform B1 field in Y-direction of $18 \mathrm{~mm}$ at more than $25 \mathrm{~mm}$ length of this volume in Z-direction. These experimental data do not contradict the results of simulations presented in Figures 2.12d and 2.12f.

Thus, both simulations and experiments confirm superior performance of the probes with cosine-profiled patches in regard of the formation of spacious sampling volume with uniform distribution of $\mathrm{B} 1$ field. 


\subsection{Comparing Patch Probes with Birdcage Coils}

In this section, B1 field homogeneity and Q-factors for patch probes, composed of patches with engineered substrates and of cosine-profiled patches, have been compared with the same characteristic parameters of birdcage coils, which are considered as "golden standard" of RF volume probes in MRI systems. The design of the birdcage coil incorporated usual quantity of eight straight conductive element (rungs), which were electrically connected by capacitors between each of neighboring rungs. To perform the comparison, the dimensions of modeled birdcage coils were adjusted so that the lengths of their rungs were equal to the lengths of patch electrodes, while the areas of coil XY cross-sections (normal to their axes) were equal to the areas of similar cross-sections of inter-patch space in patch probes. Since the latter areas in the probes composed of patches with engineered substrates could be represented by a rectangle with Y-dimension equal to the distance between patches $(30 \mathrm{~mm})$ and X-dimension defined by the patch width (about $20 \mathrm{~mm}$ ), the circular area of the inner space in the birdcage, used for comparison, was taken equal to $600 \mathrm{~mm}^{2}$ that defined the diameter of the birdcage to be of about $28 \mathrm{~mm}$. Although the lengths of birdcage rungs were taken equal to the lengths of patch electrodes, i.e. to $30 \mathrm{~mm}$, the entire length of the birdcage coil was of $40 \mathrm{~mm}$, to incorporate so-called end rings of the coil (circular conductive rings). This length determined the length of cylindrical phantom used for birdcage coil loading. At the same length of cuboid-shape phantom used in the volume probe composed of patches with engineered substrates, the phantom appeared extended beyond the patch electrode edges. However, it was found that this did not affect the response of the probe.

Most uniform B1 field within the birdcage coil was found along vertical axis passing through the coil center. Therefore, to compare the performances of the birdcage coil probe and the probe composed of patches with engineered substrates, we first investigated changes of B1 field magnitudes along Y-axes in two probes. Simulations were performed at loading both probes by lossy phantoms mimicking lung tissues ( $\varepsilon r=34, \sigma=0.75 \mathrm{~S} / \mathrm{m}$ ) [110]. Although the shapes of phantoms in two probes were different because of different shapes of inter-probe spaces, the volumes of phantoms were adjusted to be identical. 
Therefore, while the phantom for the patch-based probe had the dimensions of $14 \mathrm{~mm} \times$ $10 \mathrm{~mm} \times 40 \mathrm{~mm}$, the cylindrical phantom for the birdcage probe had the diameter of $13 \mathrm{~mm}$. As seen in Figure 2.14, the values of simulated B1 field magnitude in the patch-based probe appeared to be higher than these values in the case of birdcage probe all over the Y-axis. This result allowed for considering patch-based volume probes as competitive with birdcage coil probes.

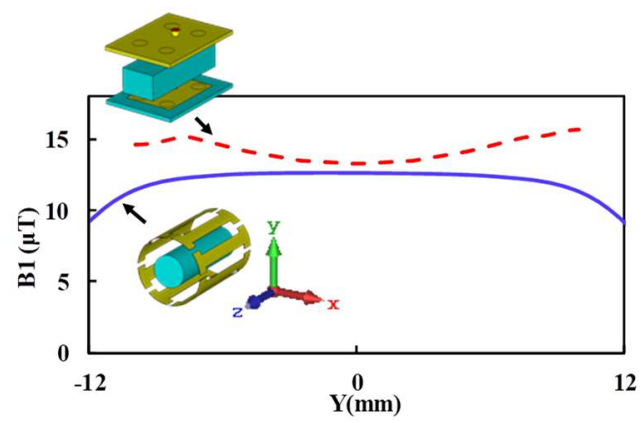

Figure 2.14. Changes of B1 field magnitudes along Y-axis passing through the centers of two probes under consideration: dashed red curve - volume probe composed of patches with engineered substrates; blue curve - birdcage probe.

Figure 2.15 compares B1 field distributions along Y-axes for the birdcage coil probe and for the probe composed of vis-à-vis placed cosine-profiled patches. In this case, the length of rungs in the birdcage coil was chosen to be of $80 \mathrm{~mm}$, i.e. equal to the length of patch electrodes in the patch probe (see section 2.4), while the diameter of coil was chosen to be $30 \mathrm{~mm}$, i.e. equal to the distance between centers of two cosine-profiled patches. Although the distributions of B1 fields in cross-sections of two probes were not identical, their difference was much less than that at previous comparison. Therefore, identical cylindrical phantoms representing lung tissues were employed in both probes. The diameters of phantoms were equal to $20 \mathrm{~mm}$ and the lengths - to $100 \mathrm{~mm}$ (equal to the entire length of the coil with end rings). It can be seen from Figure 2.15 that the probe composed of cosineprofiled patches outperformed the birdcage probe, in regards of both uniformity of B1 field distribution and the strength of B1 field magnitude all over Y-axes. 


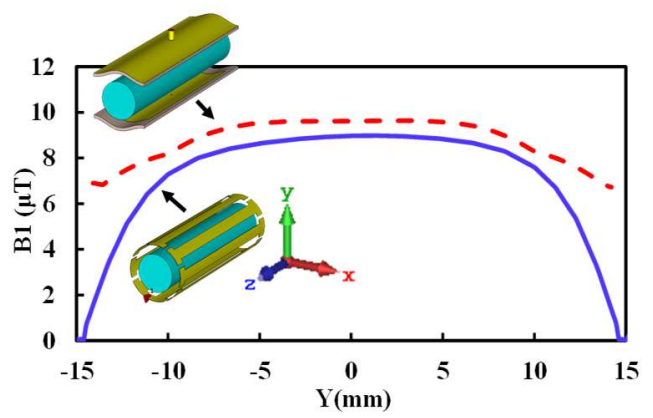

Figure 2.15 Changes of B1 field magnitudes along Y-axis passing through the centers of two probes under consideration: dashed red curve - volume probe composed of cosine-profiled patches; blue curve - birdcage probe.

Next, we compared Q-factors of patch-based probes and birdcage probes. As known, the Q-factor is defined by the ratio of energy $P$ stored in the probe and energy $L$ lost at the resonance angular frequency $\omega: Q=\omega P / L$, that makes the values of Q-factors correlating with the values of signal to noise ratio (SNR), which is critical for the resolution of images. The Q-factor could be conventionally estimated by using the ratio: $Q=\Delta f / f_{0}$, where $f_{0}$ is the resonant frequency and $\Delta f$ is the resonance bandwidth $[111,112]$. Therefore we simulated S11 spectra of the probes, found their resonance frequencies and, following [113], defined the resonance bandwidth as the frequency range, in which S11 became less than $-10 \mathrm{~dB}$ (VSWR less than 2:1). In fact, at this value of S11 it is possible to assume that the influence of reflections becomes negligible.

Figure 2.16 allows for comparing S11 spectra simulated for the volume probe composed of patches with engineered substrates and for the $40 \mathrm{~mm}$ long birdcage coil. At this comparison, the probes were not loaded by any phantoms. As seen in the figure, patchbased probe had a wider bandwidth than the birdcage coil had, so that the Q-factor of the former probe was estimated to be equal to $272(\Delta \mathrm{f}=2.3 \mathrm{MHz})$, while the $\mathrm{Q}$-factor for the birdcage coil appeared to be higher, i.e. equal to $991(\Delta \mathrm{f}=0.605 \mathrm{MHz})$.

As a possible reason of the lower Q-factor in simulated results for the probe composed of patches with engineered substrates, we assumed energy losses due to radiation. Although, unlike conventional MPAs, the patch-probe was not designed to provide efficient radiation, it still could have antenna-like properties. To check this assumption, we compared the responses of the probe with patches made on engineered substrates and of the same-size 
probe with patches on uniform substrates with effective permittivity providing resonance at $600 \mathrm{MHz}$ (this relative permittivity was found to be close to 80).

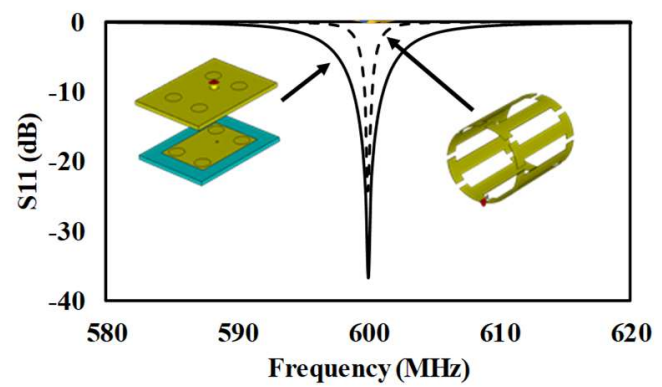

Figure 2.16 S11 spectra of two probes: the probe composed of patches on engineered substrates (left insert solid curve) and the birdcage probe with the length of $40 \mathrm{~mm}$ (right insert, dashed curve).

As seen in Figure 2.17, the probe composed of patches with uniform substrates demonstrates essentially narrower bandwidth corresponding to the Q-factor of about 750, i.e. almost three times higher than that of the probe with patches on engineered substrates. Since two probes have the same size and provide similar resonance responses, the difference in their Q-factors could be attributed to the difference in radiation losses, which are expected to be higher in patches formed on low permittivity substrates (that is just the case for patches on engineered substrates). The obtained data pointed out at importance of preventing large radiation losses at employment of patch structures in RF probes. It is obvious, that future work on RF probes composed of patches with engineered substrates should take this topic into consideration.
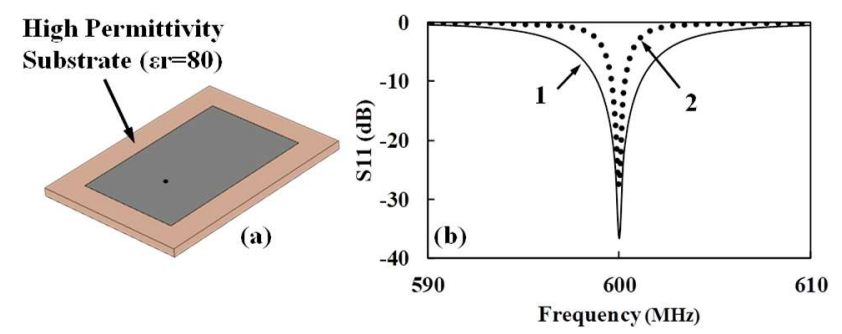

Figure 2.17 (a) Schematic of the patch with uniform substrate of high permittivity, and (b) S11 spectra of patch-based volume probes composed of patches with engineered (1-solid curve) and uniform (2-dotted curve) substrates.

In addition, we investigated how Q-factors of patch-based and birdcage coil probes were affected by loading them with phantoms. In these studies, we used the same phantoms as 
those used at investigating the difference in $\mathrm{B} 1$ field uniformity in the probe composed of patches with engineered substrates and in the birdcage probe (i.e. phantoms mimicking lung tissues with $\varepsilon r=34, \sigma=0.75 \mathrm{~S} / \mathrm{m}$ ). As seen in Figure 2.18, which presents $\mathrm{S} 11$ spectra for two probes, the response of the loaded by phantom probe composed of patches with engineered substrates did not change significantly compared to the response of unloaded probe (Figure 2.16), while loading the birdcage probe, just opposite, led to drastic changes in its response (see dashed curves in Figures 2.16 and 2.18). Correspondingly, the Q-factor of patch-based probe after loading $(\mathrm{Q}=257, \Delta \mathrm{f}=2.33 \mathrm{MHz})$ remained on the same level as that of unloaded probe $(\mathrm{Q}=272, \Delta \mathrm{f}=2.3 \mathrm{MHz})$, with the changes not exceeding several percent. In contrary, the $\mathrm{Q}$-factor of the birdcage coil decreased at loading from $991(\Delta \mathrm{f}=$ $0.605 \mathrm{MHz})$ to $428(\Delta \mathrm{f}=1.4 \mathrm{MHz})$, i.e. dropped down by more than $50 \%$.

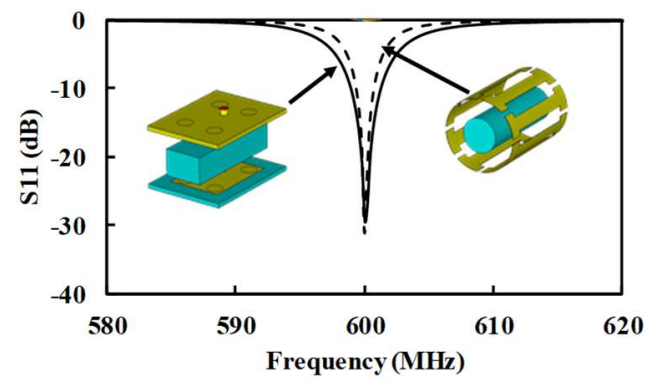

Figure 2.18 S11 spectra of compared in Figure 2.16 probes after loading them by phantoms used at obtaining the data presented in Figure 2.14. Left insert and solid curve represent the probe composed of patches with engineered substrates, while right insert and dashed curve represent the $40 \mathrm{~mm}$ long birdcage coil.

In contrast with the probe composed of patches on engineered substrates (Figure 2.16), the resonance response of the probe composed of cosine-profiled patches demonstrated very narrow bandwidth (Figure 2.19). Such difference in responses of two probes could be related to the difference in radiation efficiency of respective patches. In fact, cosineprofiled patches were designed using uniform substrates with the relative permittivity of 10 , i.e. more than twice higher than that of host material in engineered substrates (see section 2.4). It had to lead to lower radiation efficiency. In addition, cosine-profiled patches had the lengths of electrodes substantially exceeding their widths that was also not in favor of high radiation. 


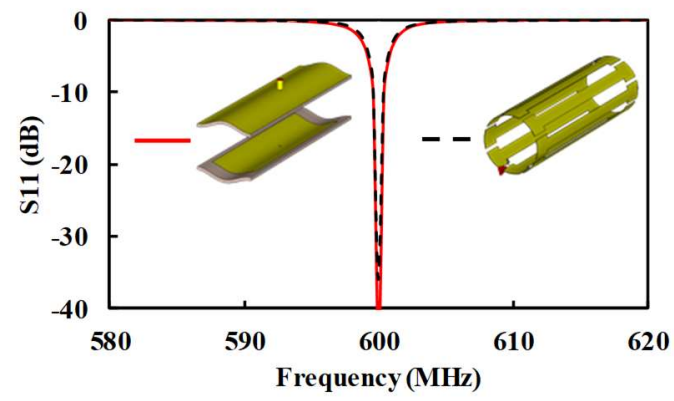

Figure 2.19 S11 spectra of volume RF probes composed of cosine-profiled patches (red solid curve) and of properly elongated birdcage probe (dashed curve).

As seen in the Figure 2.19, the curves, representing resonance responses of the volume probe composed of cosine-profile patches and of the birdcage coil with respectively increased up to $100 \mathrm{~mm}$ length, almost coincide. The bandwidths of resonance responses in both probes did not exceed $0.8 \mathrm{MHz}$, and the Q-factors of these probes were of about 750. This result allows for concluding that volume probes composed of cosine-profiled patches are well competitive with birdcage coil probes in relation to radiation losses. If the uniformity and the strength of B1 fields in new probes are taken into account, as well as the simplicity of their design and the possibility of extending the frequency range of their operation up to high microwave range, the proposed patch-based volume probes can be considered as superior and perspective option.

In addition, loading the volume probe composed of cosine-profiled patches by the same phantom, as that used at the studies of B1 field uniformity within the probes and obtaining the data presented in Figure 2.15, did not lead to drastic changes of the probe resonance response (see red solid curves in Figures 2.19 and 2.20). The Q-factor of the probe composed of cosine-profiled patches experienced relatively small changes from 750 in unloaded case down to 700 at loading, i.e. decreased by less than 7\%. In contrast, loading the comparable birdcage coil led to strong increase of the response bandwidth and, correspondingly, to the deep drop of the Q-factor value. From comparison of the data presented in Figures 2.19 and 2.20, it follows that after loading the birdcage coil by the phantom, its Q-factor changed from 750 down to 240, i.e. became of about three times less. The revealed difference in changes of probe Q-factors after loading probes by phantoms 
demonstrates an important advantage of the newly developed volume probes composed from cosine-profiled patches.

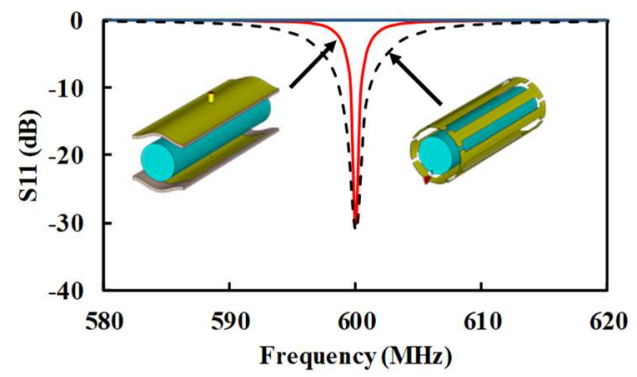

Figure 2.20 S11 spectra of compared in Figure 2.19 volume probes after loading them by phantoms used at obtaining the data presented in Figure 2.15. Left insert and solid curve represent the probe composed of cosine-profiled patches, while right insert and dashed curve represent the $100 \mathrm{~mm}$ long birdcage coil.

The results of Q-factor investigation for various unloaded and loaded by phantoms probes are also combined in Table 2.1.

Table 2.1 Comparison of calculated q-factors of proposed volume patch probes and birdcage coils

Q-factor

\begin{tabular}{ccc}
\hline RF probe & Unloaded & Loaded \\
\hline Birdcage (Length=40mm) & 991 & 428 \\
\hline Miniaturized engineered Patch & 272 & 257 \\
\hline Birdcage (length=80mm) & 750 & 240 \\
\hline Cosine-profiled Patch & 750 & 700 \\
\hline
\end{tabular}

As seen in the table, the birdcage coils appear extremely sensitive to their loading by phantoms and unable to keep high Q-factors at operating with lossy samples, while Qfactors of patch-based volume probes (either with engineered substrates or with cosineprofiled ones) demonstrate stability at loading. These results confirm expected screening effects of patch electrodes on resonance processes in substrate cavities of microstrip patch structures, which were chosen for employment in RF probes for UHF MRI scanners.

\subsection{Conclusion}

We have demonstrated, using full-wave electromagnetic analysis and experiments in the VARIAN 14.1T MRI scanner, an opportunity to employ patch structures, inspired by the 
designs of microstrip patch antennas, to create RF probes for UHF MRI systems. We have shown that magnetic fields, formed above these structures by resonance currents in patch electrodes, can be used as B1 fields in MRI studies. Different options for controlling the strength and the uniformity of these fields have been investigated, in particular, by engineering dielectric substrates using specifically located plugs with higher permittivity and by modifying the shape of structures using properly profiled substrates. Single patch structures are shown to perform as surface coils, while vis-à-vis placed patches fed with $180^{\circ}$ phase delay can act as volume probes. Such probes provided additivity of magnetic fields formed by two patch structures in inter-patch space, while electric fields in this space appear out of phase and suppress each other that prevents their harmful effects on conductive samples under study. Comparison of patch-based probes with properly sized birdcage-based probes, considered as "golden standard" of RF volume probes for MRI systems, has shown that patch-based probes can compete with birdcage coils in respect to strength and uniformity of B1 fields. In addition, they can demonstrate in unloaded state high Q-factors comparable to those of birdcage coils, while in difference from the latter, do not lose the quality of resonances at their loading by lossy phantoms. The importance of this advantage is defined by the correlation between the probe Q-factors and the SNR values, which define image resolution at MRI studies. While the complexity of birdcage coils increases with the increase of operation frequency, patch-based probes are expected to provide advantageous performance up to higher microwave range at relative simplicity of their implementation. 


\section{Clarifying the nature of negative refraction in all-dielectric metamaterials composed of identical resonators}

\subsection{Introduction}

To achieve the goal of this Chapter it was necessary to investigate the roles of Mie resonances and dispersive phenomena on wave propagation through arrays of identical dielectric resonators (DRs), which are considered in current literature as all-dielectric metamaterials (adMMs). To do that we had to compare spectra of Mie responses in DRs and other data about appearance of resonances with spectral characteristics of array dispersion responses. In particular, it was desirable to analyze spectra of array effective parameters, which could be extracted from the scattering parameters spectra. Calculations of Mie responses were possible only for spherical and cylindrical resonators. Therefore, following most of the studies of resonant dielectric MMs we investigated arrays of infinitely long dielectric rods. As in many other works, TM excitation was used in simulations. The dimensions of rods and the lattice parameters of arrays were used to correspond the conditions in most advanced studies of adMMs. In particular, the basic part of our studies was done for arrays, parameters of which corresponded to the parameters used in [83], where relative permittivity of rod was $\varepsilon_{r}=100$, radius $r=10 \mu \mathrm{m}$ and lattice constant $a=100 \mu \mathrm{m}$. Other part of our studies used parameters of most cited work [75] with $\varepsilon_{r}=600, r=3 \mathrm{~mm}$, and $a=10 \mathrm{~mm}$. It is worth mentioning here that both cited above works, conclusion of which were completely revised by our studies, ignored dispersive phenomena, and viewed the DR arrays as homogenized media, similar to most other works on adMMs. Therefore, spectra of effective parameters in these works were extracted from scattering data for one unit cell according to usual practice for MMs. We also conducted such extraction, however also analyzed the dispersion phenomena. The choice of such approach was defined by our intent to find a bridge between results obtained in previous studies of adMMs and numerous data about negative refraction in all-dielectric photonic crystals, which, in fact, had a lot in common with adMMs. To incorporate the dispersion phenomena in calculations of scattering spectra of arrays, we used special models, which 
are described below. To provide better insight into resonance processes and wave phenomena in adMMs, we simulated, in addition, field patterns in cross-sections of arrays.

\subsection{Modelling of DR arrays}

At simulations of EM responses, we employed two types of modelling DR arrays:

1- One cell models (Figure 3.1a), which are the basic ones at the analysis of responses from conventional homogenized MMs. These models exclude any account for array periodicity along the wave propagation direction.

2- The models represented by the row of cells stacked in the direction of wave propagation. At periodic boundary conditions in two perpendicular to the k-vector directions, these models allow for accounting for the effects of array periodicity and for investigating dispersion phenomena in PhC structures. We usually considered the rows of 5 cells, as according to [114], this quantity is enough to demonstrate the specifics of the $\mathrm{PhC}$ response (Figure 3.1b). However, for studying more specific effects, models comprised of more than 5 cells were also employed.

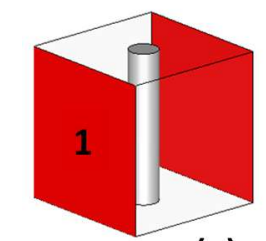

(a)

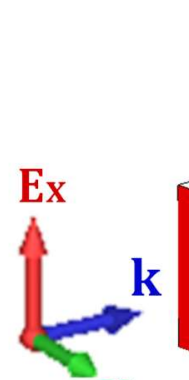

Hy

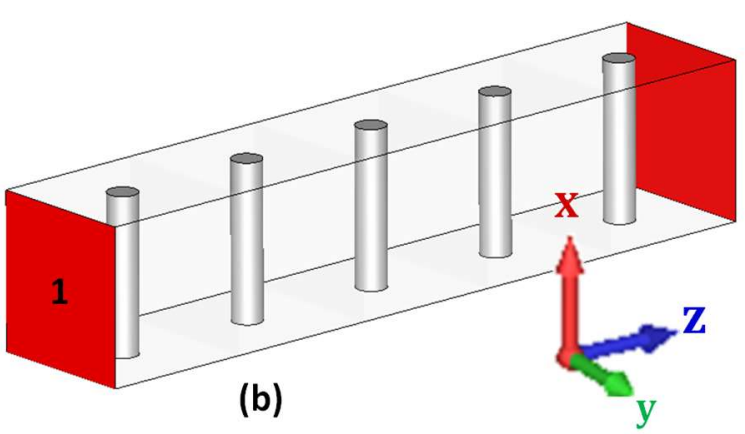

(b)

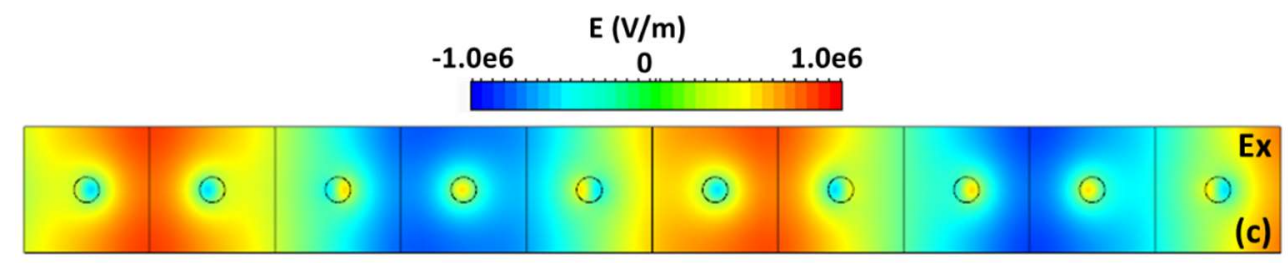

Figure 3.1 (a) One-cell model of adMM, (b) model of 5 stacked cells, (c) snap-shot of E-field intensity at wave propagation through a 10 -cell model of adMMs comprised of rods with $\mathrm{r}=10 \mathrm{~nm}$ and $\mathrm{a}=100 \mathrm{~nm}$ at sampled at $\mathrm{f}=1.28 \mathrm{THz}$. (Fig. 1 in ref. [115], (C) 2017 IOP Publishing. Reproduced with permission. All rights reserved. See Appendix A for documentation of permission to republish this material.)

CST Microwave Studio and COMSOL Multiphysics have been used as two strong fullwave software packages for studies of S-parameter spectra, field intensity distributions and 
wave patterns in 1-cell and rows of multiple cells. The snapshots of field intensity distributions in the rows of cells at wave propagation through the array (Figure 3.1c) could be used to estimate the values of refractive indices, which characterize wave propagation. For this purpose, we determined graphically the wavelengths of passing waves represented by snapshots $\left(\lambda_{\text {est }}\right)$ and then found the absolute index values by using relation: $n=c_{0} /\left(f \lambda_{\text {est }}\right)$, where $c_{0}$ is the speed of light in free space and $f$ is the frequency at which we took the snapshot of the wave pattern. Looking at the snapshot presented in Figure 3.1c it can be concluded that the depicted snapshot represents the wavelength of 470 microns. Such wavelength corresponds to the index value of 0.5 . The same value can be obtained by using the retrieval procedure applied to scattering parameters data for the row of cells at the frequency of $1.28 \mathrm{THz}$ (shown below in Figure 3.2d). The retrieval procedure utilizing S-parameter spectra was the basic one in this work for obtaining spectra of index values. For this procedure, we usually employed S-parameter spectra simulated for 5-cell models. However, one-cell models with relative permittivity values of $\varepsilon_{r}=100$, and $\varepsilon_{r}=600$, were also checked for comparison with the results in [83] and [75], respectively. It should be mentioned that the index spectra for two types of models (one cell and five cells) did not differ too much. Index retrieval was based on employing the equation given in [22]:

$$
n=\frac{1}{k_{0} d}\left[\left\{\left[\ln \left(e^{j k_{0} n d}\right)\right]^{\prime \prime}+2 m \pi\right\}-i\left[\ln \left(e^{j k_{0} n d}\right)\right]^{\prime}\right]
$$

where symbols $(\cdot)^{\prime}$ and $(\cdot)^{\prime \prime}$ denote the real and imaginary part of the refractive index, respectively, and ' $m$ ' is an integer number called branch number, $e^{j k_{0} n d}=\frac{S_{21}}{1-\Gamma S_{11}}, \Gamma=\frac{z-1}{z+1}$, and $z= \pm \sqrt{\left(1+S_{11}\right)^{2}-S_{21}^{2} /\left(1-S_{11}\right)^{2}-S_{21}^{2}}$.

It is challenging to choose the correct value for the branch number, ' $m$ ' in (3.1), because this coefficient has to be changed, for example, from 0 to 1 , to prevent the discontinuity in the index spectra [22]. However, we preferred to follow [23] and avoid such changes of coefficient ' $m$ ', and use $m=0$ for the entire spectrum. For index determination, we constantly controlled obtained index values by employing alternative options such as estimating wavelength on wave pattern in the cases of doubts about the accuracy of calculated index results and followed $[116,117]$ to account for the effect of array 
periodicity on index spectra.

Mie coefficients spectra for the rods used in DR arrays were calculated by using the expression for Mie scattering coefficients from [74]:

$$
b_{n}=\frac{\sqrt{\varepsilon_{r}} J_{n}\left(k_{0} r\right) J_{n}^{\prime}\left(\sqrt{\varepsilon_{r}} k_{0} r\right)-J_{n}\left(k_{0} r\right) J_{n}^{\prime}\left(\sqrt{\varepsilon_{r}} k_{0} r\right)}{\sqrt{\varepsilon_{r}} H_{n}\left(k_{0} r\right) J_{n}^{\prime}\left(\sqrt{\varepsilon_{r}} k_{0} r\right)-H_{n}^{\prime}\left(k_{0} r\right) J_{n}\left(\sqrt{\varepsilon_{r}} k_{0} r\right)}
$$

where $k_{0}$ is wavenumber in free space, $r$ is the radius, and $J_{n}$ and $H_{n}$ represent Bessel functions of the first kind and Hankel function of the second kind, respectively, and Lagrange's notation $(\cdot)^{\prime}$ is used here to denote first derivatives of these functions.

\subsection{Mie resonances versus surface resonances in rod arrays}

Figure 3.2 shows a set of spectra, described above in Section 3.2, characterizing the adMM represented by a square lattice of rods with $\varepsilon_{r}=100, r=10 \mu \mathrm{m}$ and, $a=100 \mu \mathrm{m}$. Similar structure was investigated in [83]. The latest editions of MPB software [18] was used for calculation of dispersion diagrams.

As seen in Figure 3.2a, the dispersion diagrams for arrays under study demonstrate two branches independent on K-vector, at frequencies $1.18 \mathrm{THz}$ and $1.82 \mathrm{THz}$, which are close to characteristic frequencies for coefficients $\left|b_{1}\right|$ and $\left|b_{2}\right|$ in Figure 3.2b. These coefficients correspond to the magnetic and higher order Mie resonances, respectively [74], for which the simulated electric and magnetic field patterns are shown in inserts above Figure 3.2b. These straight branches were also seen in [118] and called localized bands. Interestingly, no localized band related to the electric-type Mie resonance were observed neither in our studies, nor in [118]. It is seen in Figure 3.2b that the electric Mie resonance represented by the coefficient $\left|b_{0}\right|$ can be related to the higher frequency edge of fundamental band. 

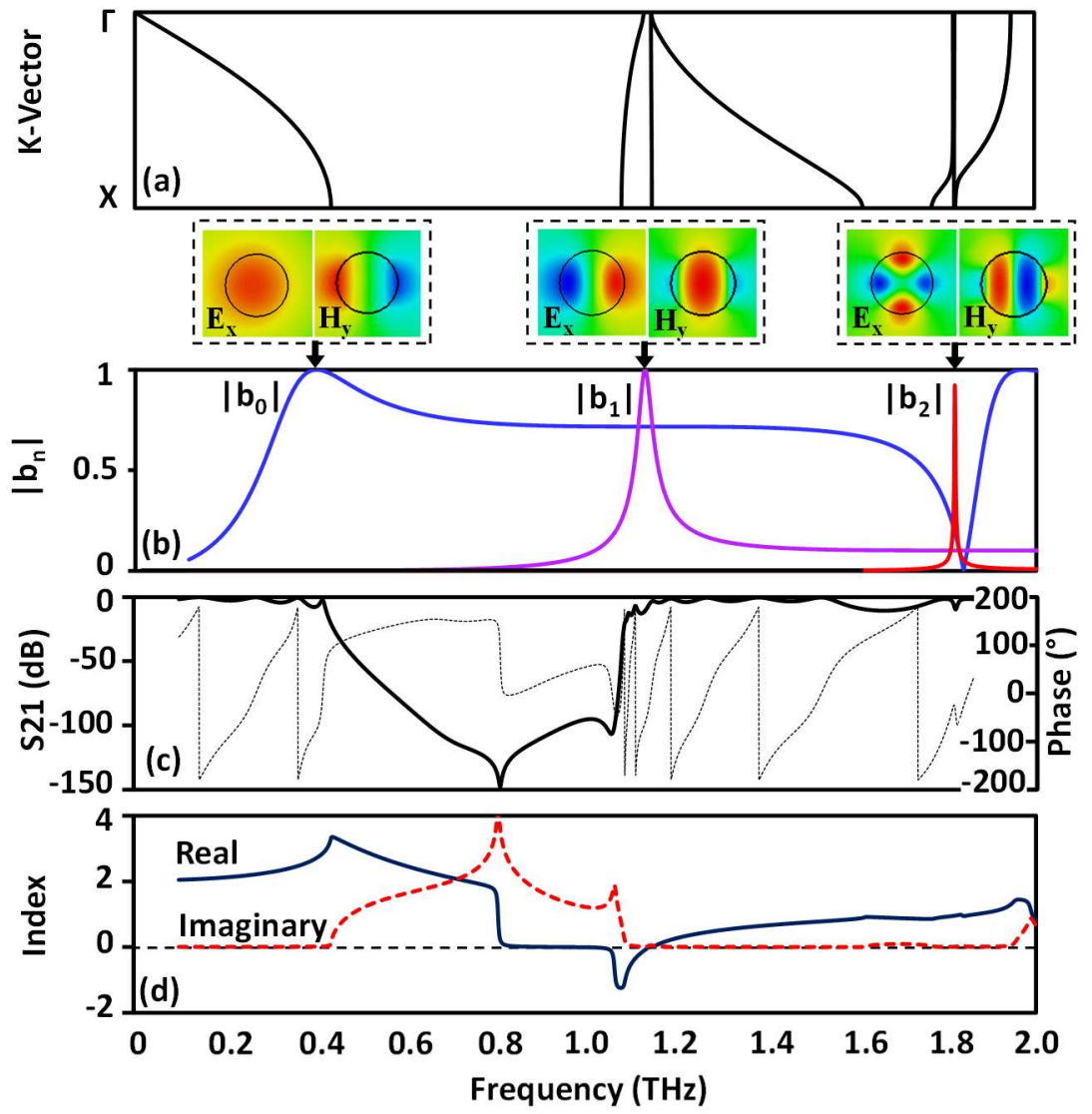

Figure 3.2 Spectra of (a) dispersion diagram for infinite PhC, (b) Mie scattering by a single rod with inserted field patterns at peaks, (c) S21 magnitude (solid line) and phase (dashed line) for the model of five cells in a row, (d) index components for one-cell model, when $\varepsilon \mathrm{r}=100, \mathrm{r}=10 \mu \mathrm{m}$, and $a=100 \mu \mathrm{m}$. (Fig. 2 in ref. [115], (C) 2017 IOP Publishing. Reproduced with permission. All rights reserved. See Appendix A for documentation of permission to republish this material.)

Figures 3.3a and 3.3b present, respectively, E- and H-field distribution in the array crosssection at the frequency of fundamental band edge. Electric and magnetic field patterns in each cell look corresponding to those at the first insert above Figure 3.2b, which were observed at the electric Mie resonance in a single rod. However, the phases of resonances in neighboring cells look shifted by $180^{\circ}$. It means that Figures $3.3 \mathrm{a}$ and $3.3 \mathrm{~b}$ represent the 'odd' transmission mode at the edge of the fundamental band. 

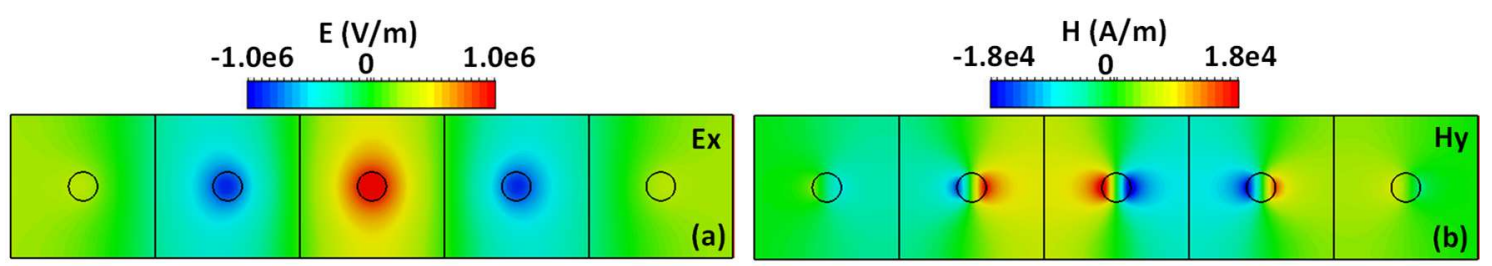

Figure 3.3 E- and H-field patterns in the cross-section of 2D rod array: (a)-(b) at $0.42 \mathrm{THz}$, the edge frequency of fundamental band. (Fig. 3 in ref. [115], (C) 2017 IOP Publishing. Reproduced with permission. All rights reserved. See Appendix A for documentation of permission to republish this material.)

From Figure 3.3 it can be also seen that field magnitude is changing from cell to cell along the chain with maximal fields observed in the median cell that is typical for Fabry-Perot (F-P) resonances in PhCs fragments of restricted lengths [114]. As known, F-P resonances are accompanied by formation of standing waves, and snapshots presented in Figures 3.3a and $3.3 \mathrm{~b}$ look corresponding to the half-wavelength standing wave. It should be noted that out-of-phase arrangement of neighboring Mie resonances excludes a possibility of their effect on effective medium parameters, and, hence, excludes any possible contribution of the electric resonance in double negativity of effective parameters.

S21 spectrum of the respective rod array in Figure 3.2c demonstrates two dips at $0.8 \mathrm{THz}$ and $1.05 \mathrm{THz}$. These dips are assumed to be related to Mie resonances according to [83]. However, these frequencies are different from Mie resonant frequencies seen in Figure 3.2b. Moreover, by comparing the $\mathrm{S} 21$ spectra and the dispersion diagram, it can be seen that the dips are located within the bandgap and thus, can be related only to resonances caused by evanescent waves. As seen in Figure 3.4, presenting field patterns at S21 dips, waves do not propagate through the array at frequencies of the dips and penetrate only in the first layer formed by edge cells. The magnitude of the field in these cells is also much less than that expected at the resonances that allows for relating the dips to surface phenomena, which do not extend inside the array volume. 


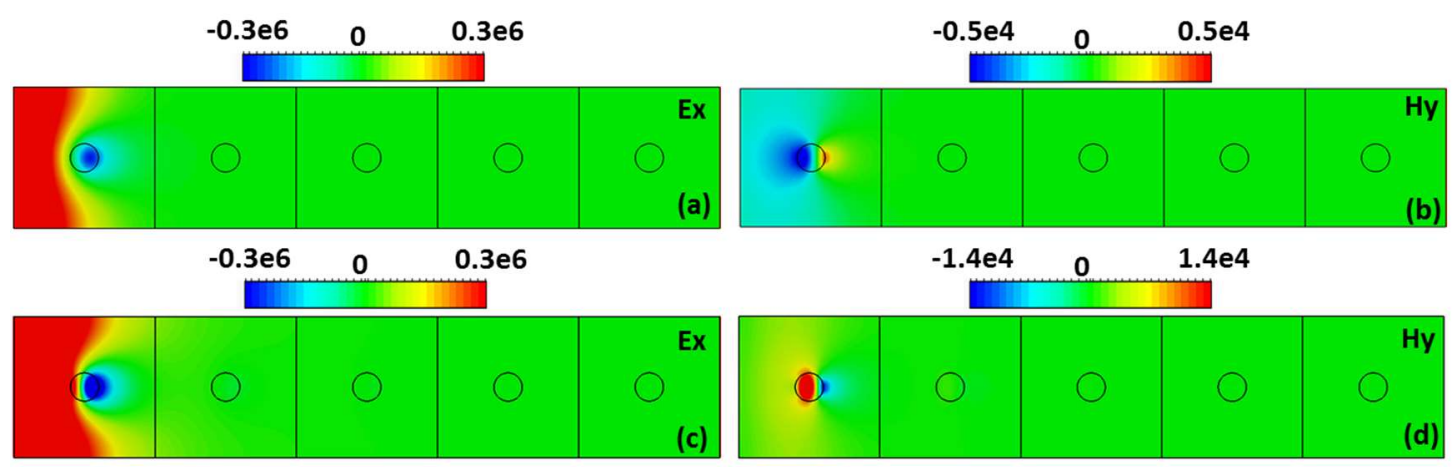

Figure 3.4 E- and H-field patterns in the cross-section of 2D rod array: (a),(b) at the $1^{\text {st }}$ dip in Figure 3.2c $(0.8 \mathrm{THz})$ in S21 spectrum; (c),(d) at the $2^{\text {nd }} \operatorname{dip}(1.05 \mathrm{THz})$ in S21 spectrum. (Fig. 3 in ref. [115], (C) 2017 IOP Publishing. Reproduced with permission. All rights reserved. See Appendix A for documentation of permission to republish this material.)

It is not excluded that distorted fields in the rods of the first layer can be related to interaction between electric and magnetic Mie resonance modes in surface cells, since the frequency ranges, in which imaginary components of index at two S21 dips are defined, are seen overlapped in Figure 3.2d.

\subsection{Correlation between changes of index spectra and of dispersion diagrams at decreasing the lattice constant of arrays}

For investigating the correlation between index spectra and the dispersion diagrams, we had to consider only those parts of index spectra, over which the imaginary component remained equal to zero, thus providing the conditions for wave propagation. As in [83] at lattice constant $a=100 \mu \mathrm{m}$, extracted by us index spectra showed a region with the negative real value below $1.1 \mathrm{THz}$ (Fig. 3.2d). To understand the reasons of this phenomenon, we studied changes of dispersion diagrams and index spectra at decreasing the lattice constant from $120 \mu \mathrm{m}$ to $80 \mu \mathrm{m}$. As seen in Figures 3.5a and 3.5b, at $a=120 \mu \mathrm{m}$ the real index value is positive near $1 \mathrm{THz}$ in a wide frequency range from $0.95 \mathrm{THz}$ to $1.07 \mathrm{THz}$. After decreasing the lattice constant to $a=110 \mu \mathrm{m}$, real index values still remained positive, however, in a narrower band between $1.04 \mathrm{THz}$ and $1.1 \mathrm{THz}$. Further decreasing the lattice constant down to $a=105 \mu \mathrm{m}$ led to disappearance of positive band with a trend of switching indices to negative values. As can be seen in Figures 3.5g and 3.5f, at $a=100 \mu \mathrm{m}$, the band 
with negative real component of index is formed from $1.08 \mathrm{THz}$ to $1.12 \mathrm{THz}$. At $a=90 \mu \mathrm{m}$ this band becomes extended from $1.08 \mathrm{THz}$ to $1.17 \mathrm{THz}$ and remains almost unchanged at $a=80 \mu \mathrm{m}$.
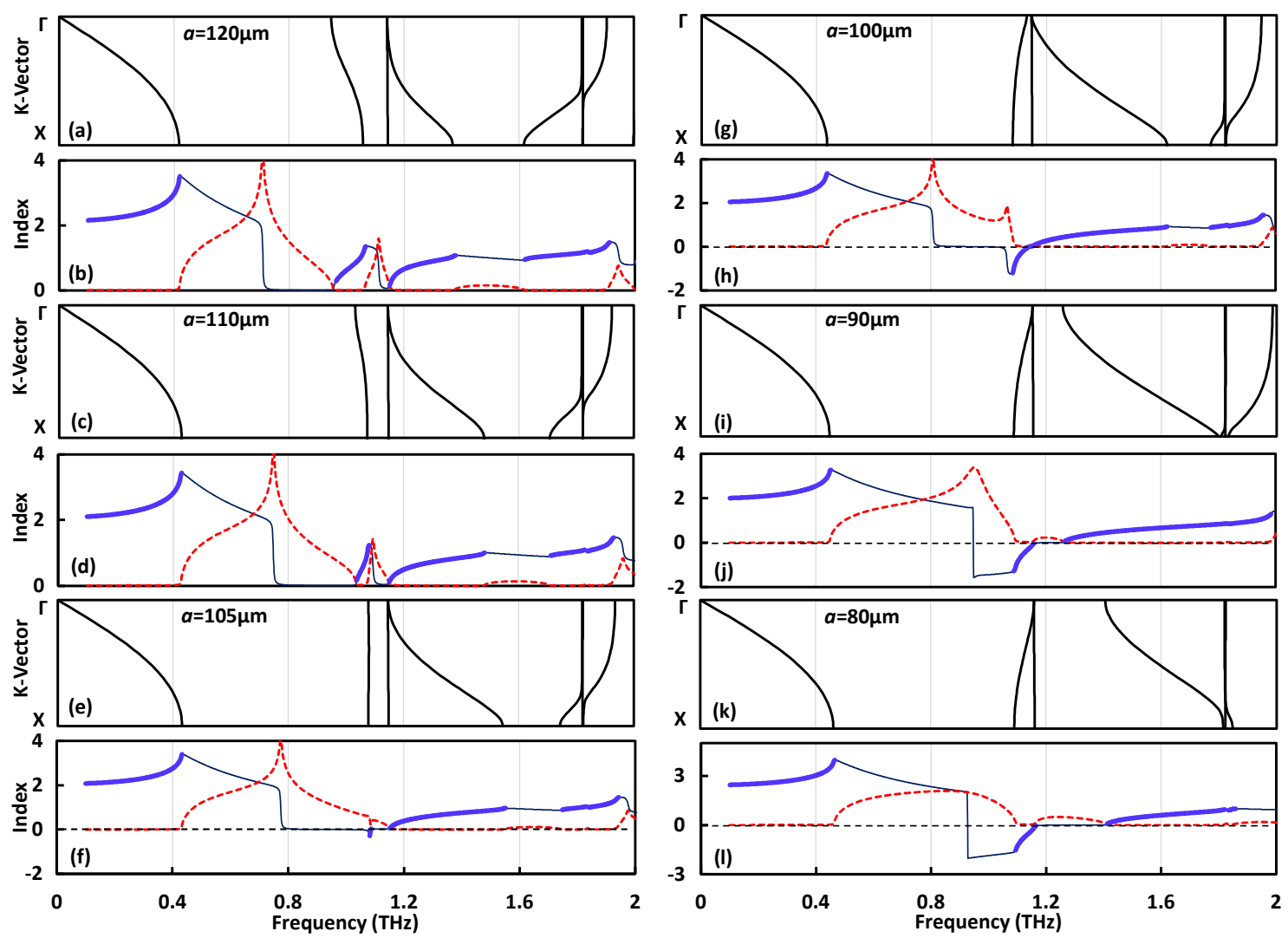

Figure 3.5 Dispersion diagrams and real (solid blue) and imaginary (dashed red) components of refractive indices for 2D rod arrays ( $(\mathrm{r}=100$ and $\mathrm{r}=10 \mu \mathrm{m}$ ) at various array lattice constants, $a$, (a) and (b) $120 \mu \mathrm{m}$, (c) and (d) $110 \mu \mathrm{m}$, (e) and (f) $105 \mu \mathrm{m}$, (g) and (h) $100 \mu \mathrm{m}$, (i) and (j) $90 \mu \mathrm{m}$, (k) and (l) $80 \mu \mathrm{m}$. (Fig. 4 in ref. [115], (C) 2017 IOP Publishing. Reproduced with permission. All rights reserved. See Appendix A for documentation of permission to republish this material.)

It can be seen in Figure 3.5 that the described above frequency ranges of real positive and real negative index values exactly correspond to the location of the $2^{\text {nd }}$ transmission band in dispersion diagrams. It is also seen in diagrams that at $a=120 \mu \mathrm{m}$, the slope of the $2^{\text {nd }}$ transmission band corresponds to positive refraction, while at decreasing the lattice constant down to $\mathrm{a}=105 \mu \mathrm{m}$, the slope becomes flat. At further decreasing the lattice constant, the $2^{\text {nd }}$ band acquires the slope associated with the negative refraction. Observed transition from positive refraction in the $2^{\text {nd }}$ transmission band in arrays with $a>105 \mu \mathrm{m}$ 
to negative refraction in arrays with smaller lattice constants could be, possibly, related to increased interaction between closer located resonators.

Since the slope of the $2^{\text {nd }}$ branch in the dispersion diagram appeared correlated with the sign and the values of refractive index at respective frequencies, we had to consider it in more details. It could be noticed that the $2^{\text {nd }}$ branches near points $\mathrm{X}$ of the dispersion diagrams seem parallel to the branch at $a=105 \mu \mathrm{m}$, which is independent on k-vector. The shape of this branch is comparable to the localized state which, as shown in Figure 3.2, is related to the magnetic Mie resonance (at $1.15 \mathrm{THz}$ for $a=100 \mu \mathrm{m}$ ). However, the latter state demonstrated full transmission through the array, while the former one, similar to what was observed at $a=105 \mu \mathrm{m}$, showed very weak transmission at less than $-50 \mathrm{~dB}$ that made phase changes along the array of rods unstable. Figure 3.6 shows the field pattern at the magnetic Mie resonance (at $1.15 \mathrm{THz}$ ) and at the lower edge (X-point) of the $2^{\text {nd }}$ branch in the dispersion diagram, where the slope is the same as that at $a=105 \mu \mathrm{m}$. As can be seen in Figures 3.6a and 3.6b, there is a superluminal phase velocity and 'even' transmission mode [25] at the magnetic Mie resonance. However, as seen in Figures 3.6c and 3.6d, the obtained field patterns for the lower edge of the $2^{\text {nd }}$ transmission band corresponds to the 'odd' transmission mode with $180^{\circ}$ phase difference between neighboring rods.

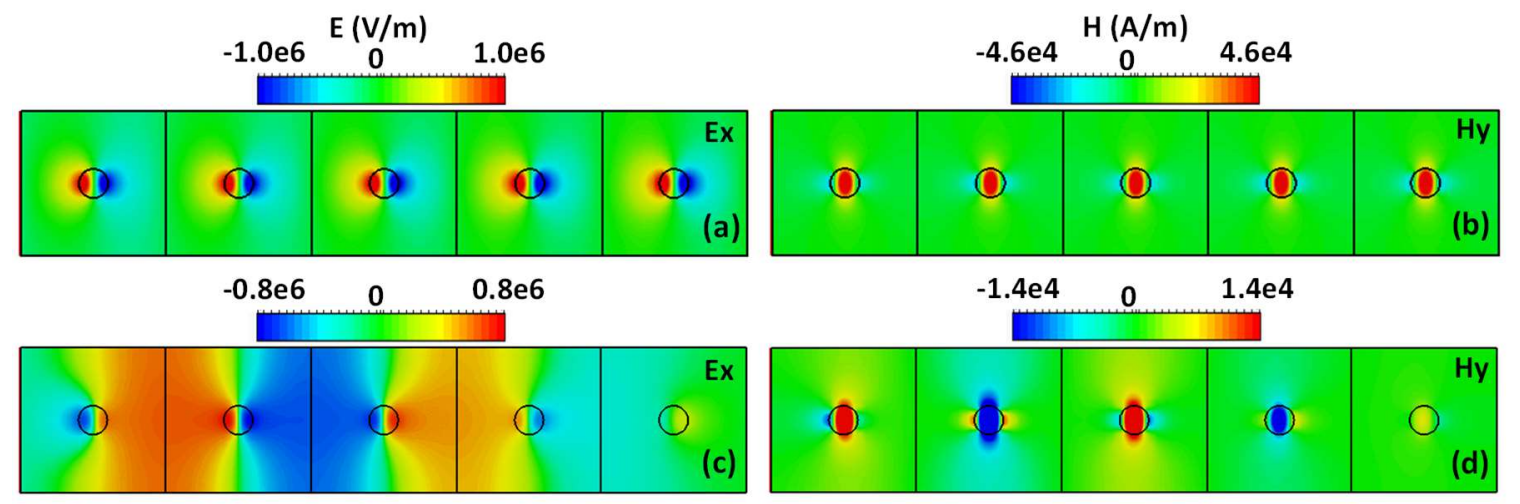

Figure 3.6 E- and H-field patterns in the cross-section of 2D rod array with a $=100 \mu \mathrm{m}$ : (a)-(b) at the frequency of localized photonic state $(1.15 \mathrm{THz})$; (c)-(d) at the lower edge of the $2^{\text {nd }}$ band in the dispersion diagram. (Fig. 6 in ref. [115], (C) 2017 IOP Publishing. Reproduced with permission. All rights reserved. See Appendix A for documentation of permission to republish this material.)

The formation of two modes for the same type of magnetic resonance could be considered as the result of splitting the resonant photonic states, due to difference in energy of 
interaction between magnetic dipoles representing resonant responses at even and odd transverse orientations [119].

Since there is a strong correlation between the $2^{\text {nd }}$ transmission branch and the sign of refractive index, we can conclude that negative refraction in adMMs is defined by specifics of their dispersive properties and not by double negativity of their effective parameters, as it is typically expected at observation of Mie resonances [120].

\subsection{Verification of extracted index spectra}

To ensure the accuracy of finding the index spectra, extracted by using the approaches corresponding to assumption that DR arrays perform as homogenized media, we could also calculate the Equi-frequency contours (EFCs), which can be used for determining the sign of refractive index $[13,121]$. Considering the array with the lattice constant $a=100 \mu \mathrm{m}$ as one of most challenging cases, we calculated the EFCs for the $2^{\text {nd }}$ and $3^{\text {rd }}$ transmission bands of its dispersion diagrams. As seen in Figure 3.7a for the $2^{\text {nd }}$ band, when $k_{x}$ or $k_{y}$ values become higher at moving along axes of the EFC graph, the frequencies of the contours crossing these axes decrease that confirms that the band can be characterized by negative indices. However, the situation is opposite for the $3^{\text {rd }}$ band. This difference is in exact correspondence with retrieved index spectra in Figure 3.4h.

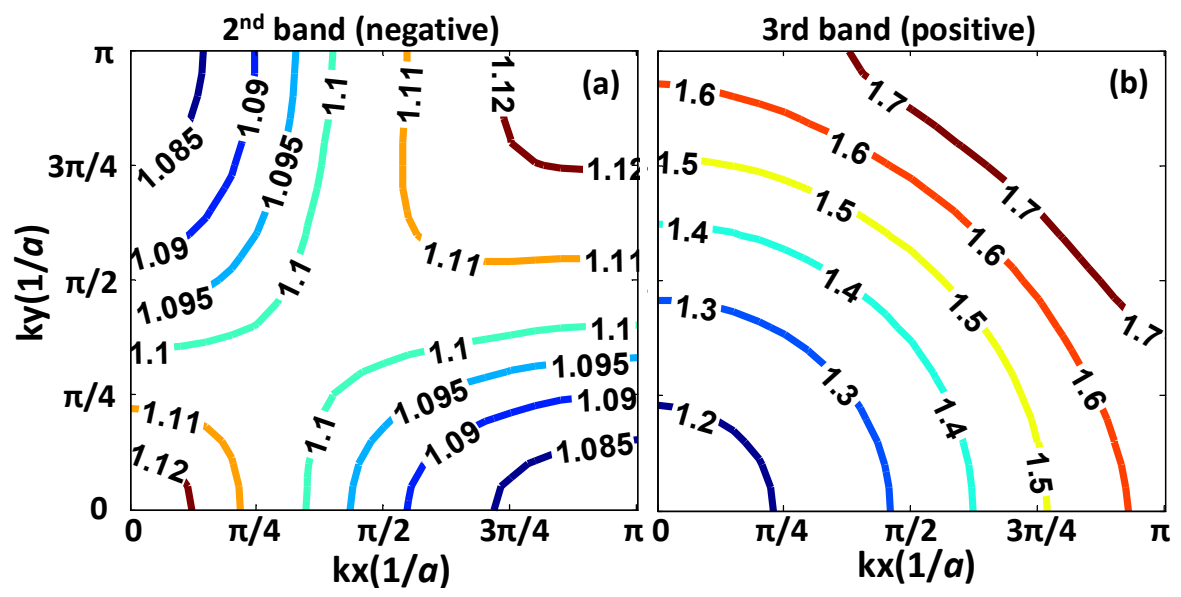

Figure 3.7 Equi-frequency contours in various frequency bands: a) 2nd band, (b) 3rd band for adMM with a $=100 \mu \mathrm{m}$. (Fig. 5 in ref. [115], (C) 2017 IOP Publishing. Reproduced with permission. All rights reserved. See Appendix A for documentation of permission to republish this material.)

We have also confirmed that the negative refraction is related to the left-handed or 
backward propagation. For this purpose, we have simulated two arrays with different quantity of cells and compared the phase changes at wave propagation along two arrays. At forward wave propagation, the longer array was expected to demonstrate a phase delay with respect to the shorter array, while at backward wave propagation, a phase advance should be expected [8]. Figure 3.8 allows for comparing phase spectra for arrays of 9 cells and of 11 cells with $a=100 \mu \mathrm{m}$. We can see that in the $2^{\text {nd }}$ band, the transmission phase of longer array advances all over the band. This behavior is specific for the backward wave propagation. However, in the $3^{\text {rd }}$ band, the delayed transmission phase confirms the forward wave propagation.

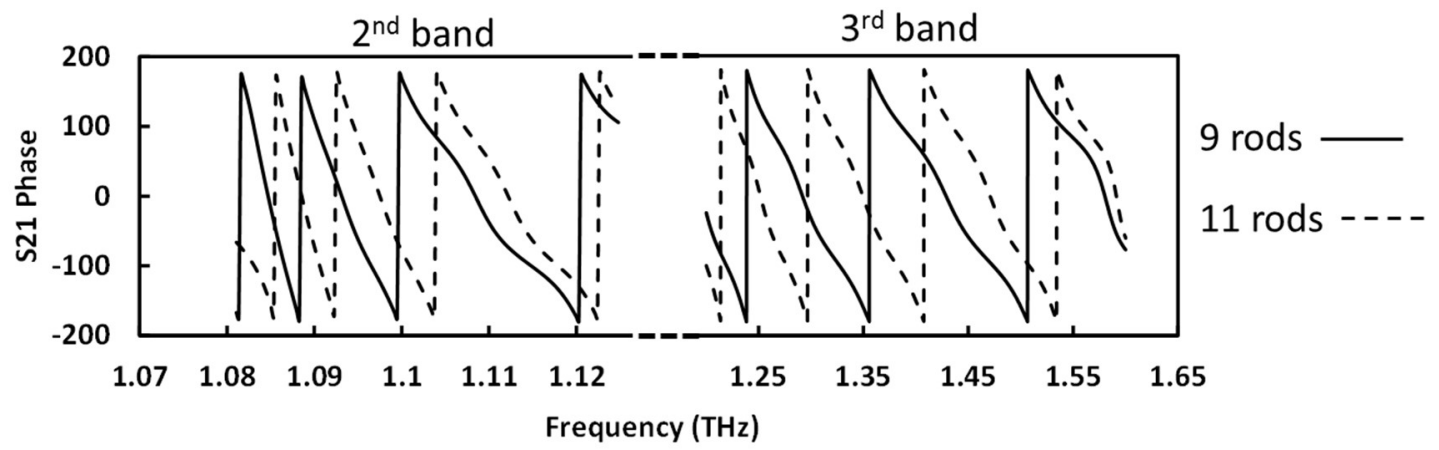

Figure 3.8 Phase change spectra at $\varepsilon_{\mathrm{r}}=100$ and $\mathrm{r}=10 \mu \mathrm{m}$, and $\mathrm{a}=100 \mu \mathrm{m}$. The model of 11 cells (dashed curves) demonstrates at the wave propagation the phase advances in the $2^{\text {nd }}$ band and phase delays in the 3 rd band against the model of 9 cells (solid curves). (Fig. 7 in ref. [115], C 2017 IOP Publishing. Reproduced with permission. All rights reserved. See Appendix A for documentation of permission to republish this material.)

\subsection{Applicability of the conclusion about the nature of negative refraction to other DR arrays}

To ensure that our conclusion about the nature of negative refraction works in other cases of DR arrays, we repeated the above-described studies for arrays used in seminal works devoted to overlapping of Mie resonances [75, 78]. The parameters of arrays in these works were $\varepsilon_{r}=600, r=0.68 \mathrm{~mm}$ and $a=3 \mathrm{~mm}$. In this section, we describe the results of comparing the band diagrams and the spectra of refractive indices for arrays with similar $\varepsilon_{r}$ and $r$, but with lattice constants varied from $15 \mathrm{~mm}$ down to $3 \mathrm{~mm}$.

As seen in Figure 3.9, there could be observed two localized bands in the dispersion diagrams of all studied arrays, the first one - at around $7 \mathrm{GHz}$ and the second one at 11 
GHz. Similar to the previous case, the localized bands were associated with the magnetic and higher order Mie resonances in all rods, while no localized states related to the electric Mie resonance were found. The real parts of index spectra observed when imaginary component of index was equal to zero appeared associated with the $2^{\text {nd }}$ transmission band, which was located in dispersion diagrams at frequencies close to $7 \mathrm{GHz}$.
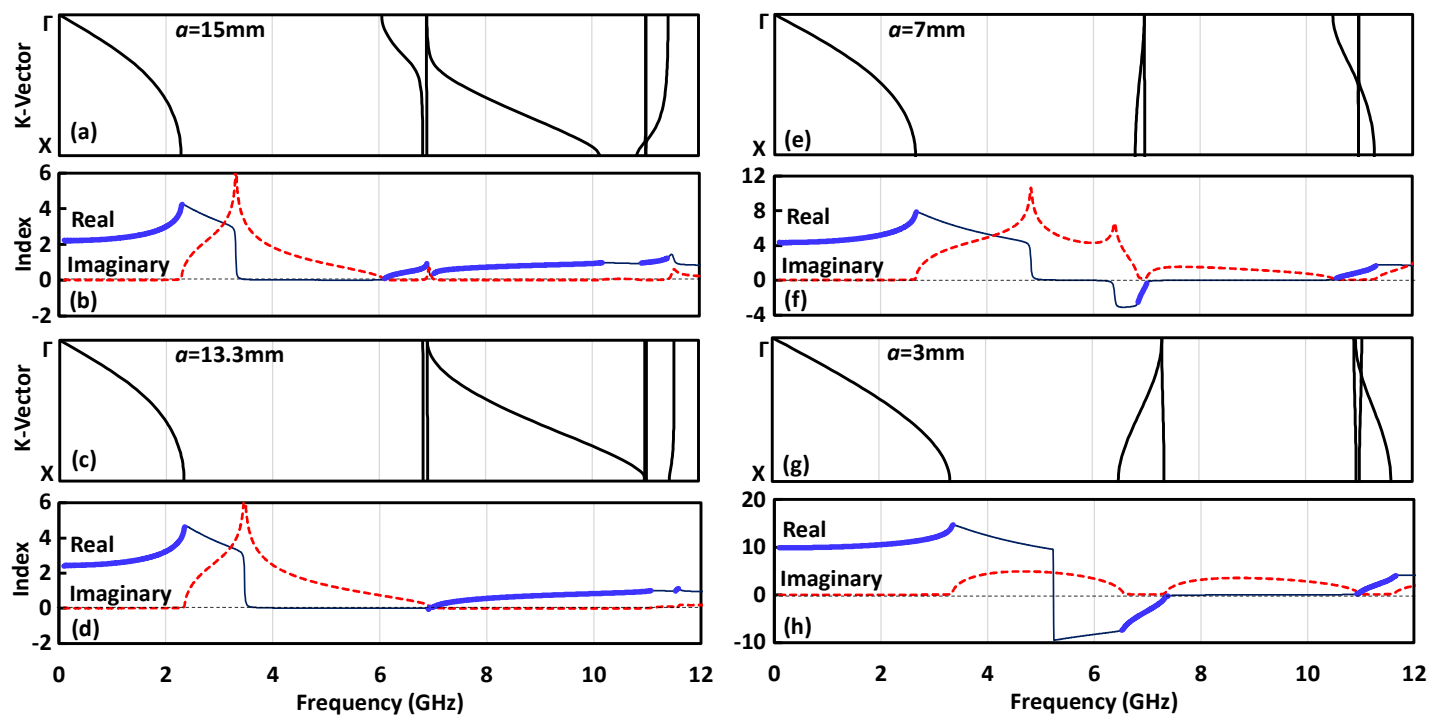

Figure 3.9 Dispersion diagrams and real (solid blue) and imaginary (dashed red) components of refractive indices for 2D rod arrays ( $\varepsilon \mathrm{r}=100$ and $\mathrm{r}=10 \mu \mathrm{m}$ ) at various array lattice constants, $a$,: (a) and (b) $15 \mathrm{~mm}$, (c) and (d) $3.3 \mathrm{~mm}$, (e) and (f) 9 mm, (g) and (h) $3 \mathrm{~mm}$. (Fig. 8 in ref. [115], (C) 2017 IOP Publishing. Reproduced with permission. All rights reserved. See Appendix A for documentation of permission to republish this material.)

From Figures 3.9 it follows that the decreasing of the lattice constant of arrays caused such changes of the branch slopes, which corresponded to gradual transformation of the sign of refractive index from positive to negative with passing through null values in between. The same changes were observed in extracted from scattering parameters index spectra. These results confirm correlation of index spectra to dispersion diagrams and thus, support our conclusion about the role of array dispersion properties in appearance of negative refraction in these arrays. 


\subsection{Conclusion}

We extracted the spectra of refraction index values from the results of simulating the scattering parameters spectra for DR arrays, considered in literature as adMMs, and compared obtained data with dispersion diagrams of these arrays. In addition, we calculated spectra of Mie resonances and simulated field patterns in array cross-sections at frequencies of these resonances and frequencies of the dips in scattering parameter spectra. The analysis of spectral characteristics of arrays has shown that negative refraction in adMMs is irrelevant to overlapping of Mie resonances and is defined by the dispersion phenomena in DR arrays at specific values of their lattice constants. 


\section{Electromagnetic responses of metasurfaces composed from dielectric nano-disk resonators}

\subsection{Introduction}

In this chapter, we describe our studies of the effects of lattice parameters of nano-disk metasurfaces on spectral positions of dipolar resonances in these structures and on their transmission spectra. These studies were undertaken to clarify the nature of full transmission through such metasurfaces at coincidence of MR and ER resonance frequencies. Revealed at our studies drastic effects of lattice parameters on spectral positions of resonances and on EM responses of metasurfaces do not allow for accepting conventional views on metasurfaces as the media, whose responses, similar to responses of MMs, are defined by the properties of single cells and are independent on inter-cell interaction and periodicity of the structures. To find out, if the dispersive phenomena affect the EM responses of metasurfaces, we calculated the dispersion diagrams for 3D arrays of nano-disks with the same parameters, as those used in metasurfaces in [95], and at similar lattice constants, and compared the specific features of responses predicted by these diagrams with characteristic features of scattering from metasurfaces at variation of disk dimensions and lattice constants. As it is shown below, the obtained data point out at an important role of dispersion phenomena, and on integration of responses from single cells due to directional coupling between them.

\subsection{Changes in spectral positions of dipolar resonances in nano-disks at varying parameters of metasurfaces.}

Figure 4.1 shows fragments of arrays under study with smallest ( $a=330 \mathrm{~nm}$ (as in [95])) and largest $(a=640 \mathrm{~nm}$ ) lattice constants. Fragments were composed of silicon disks with the radius of $120 \mathrm{~nm}$ and the refractive index of 3.5. At the smallest array (Figure 4.1a), distances between resonators were almost three times less than disk diameters, while at the

largest array (Figure 4.1b), disks were placed far from each other at the distances exceeding their diameters. The thicknesses of disks were varied in simulation between $40 \mathrm{~nm}$ to 240 
nm. A plane wave was incident along z-axis with E-field directed along $\mathrm{x}$-axis and H-field along y-axis.

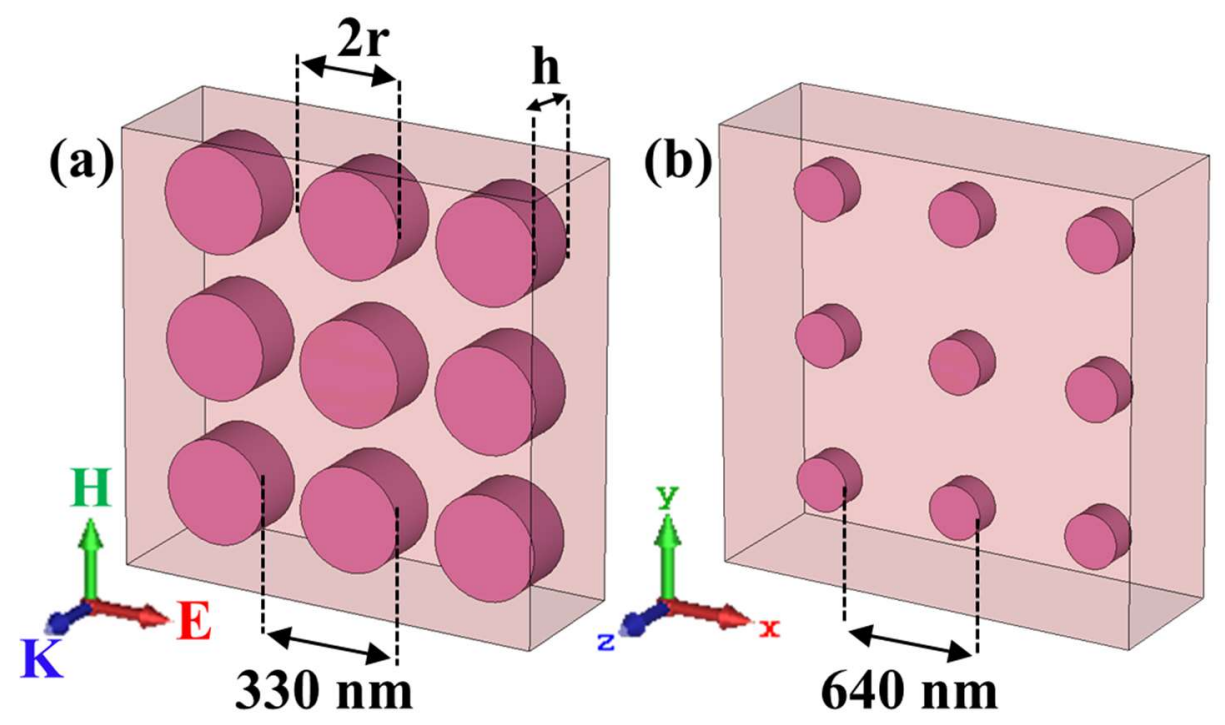

Figure 4.1 Fragments of silicon nano-disk arrays with lattice constants of (a) $330 \mathrm{~nm}$ and (b) $640 \mathrm{~nm}$. The disk thickness, h, could be varied from $40 \mathrm{~nm}$ to $240 \mathrm{~nm}$.

Figure 4.2 (two upper rows) presents changes of the resonance wavelengths at ERs and MRs at varying disk thicknesses (heights). Columns in Figure 4.2 correspond, respectively from left to right, to lattice constants $a=330 \mathrm{~nm}, 480 \mathrm{~nm}$, and $640 \mathrm{~nm}$. It is seen in Figure 4.2 that for all lattice constants, decreasing the disk thickness causes shrinking of the bandwidth of resonances and increasing their strength. However, at too small values of $h$, the former effect leads to disappearance of MRs, especially for large lattice constants. The left column in Figure 4.2 presenting the data for metasurfaces with lattice constant of about $330 \mathrm{~nm}$ shows that at large disk thicknesses, two resonances are located far from each other on frequency scale (the scale of wavelengths). Correspondingly, the S21 spectra, presented in the $3^{\text {rd }}$ row of Figure 4.2 demonstrate two deep drops looking as dark blue areas. At decreasing the disk thicknesses resonances become closer to each other and tend to coincide at $h$ around $90-120 \mathrm{~nm}$. The coincidence of resonance frequencies looks accompanied by full transmission (S21 approaches 1). Further decreasing of the disk thickness leads to restoration of two drops in S21 spectra, however in this case they look located nearby on frequency scale. It is worth mentioning that in the range of thicknesses 
$h<80 \mathrm{~nm}$, the order in which ER and MR appear in the spectra is reversed so that MR occurs at longer wavelenghs, i.e. at lower frequencies than ER. Although these data confirm the results in $[15,95]$, there are serious doubts about compatibility of field distributions characterizing two dipolar resonances in dielectric particles.
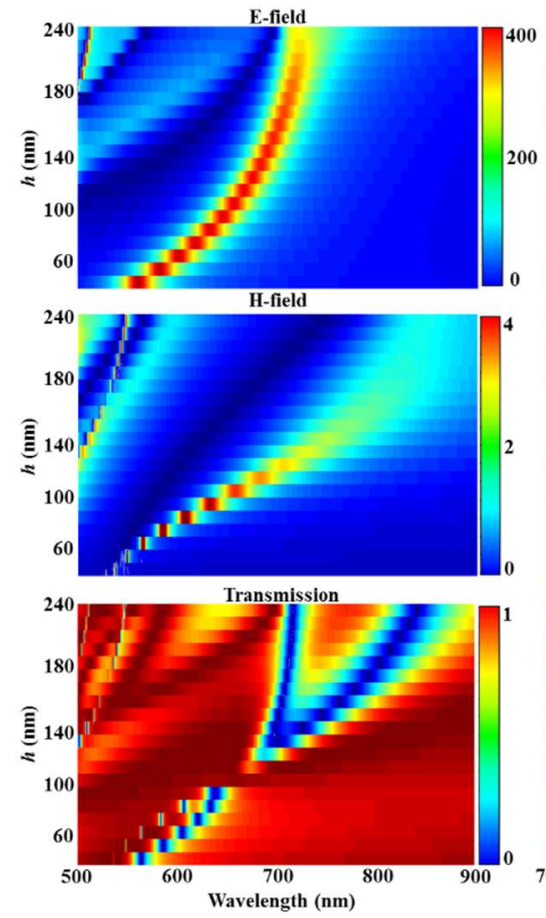
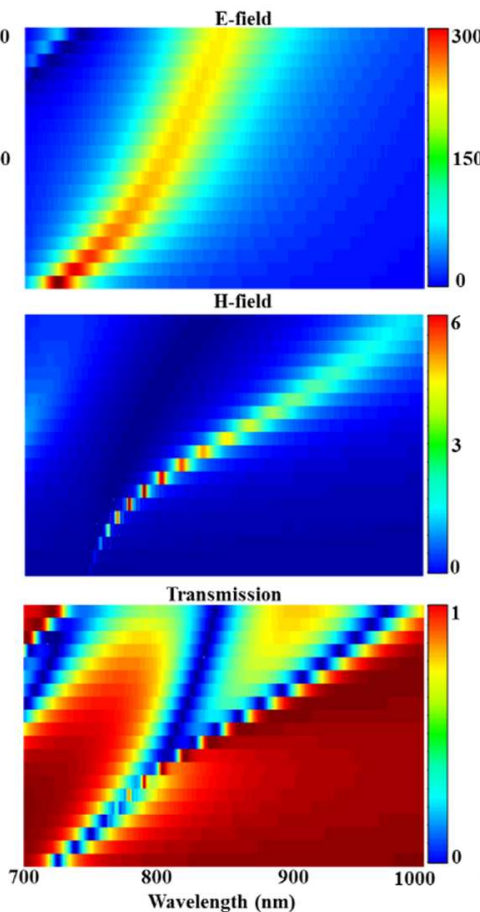
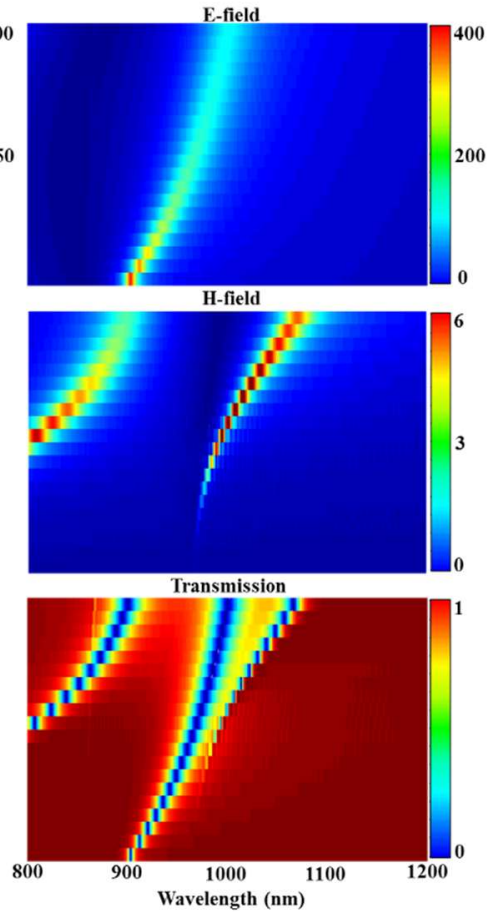

Figure 4.2 Dependencies on disk thicknesses for spectral positions of ER (upper row), MR (2nd row), and color scaled S21 spectra (lower row) at array lattice constants $\alpha$ in nm: 1st column $-330,2$ nd column -480 , and 3 rd column -640 . Disk radius is $120 \mathrm{~nm}$.

Data presented in $2^{\text {nd }}$ and $3^{\text {rd }}$ columns in Figure 4.2 illustrate complications of described above phenomena in metasurfaces with bigger lattice constants. In fact, at $a=480 \mathrm{~nm}$, spectral positions for ER and MR do not cross, and no wideband full transmission is seen when resonances come closer to each other in the spectrum. At $a=480 \mathrm{~nm}$ and $h=100 \mathrm{~nm}$, there can be seen only narrow spots with enhanced transmission within the channel related to ER. For better demonstration of the difference between these arrays and arrays with small lattice constant, we show in Figure 4.3 calculated for one cell model of metasurface spectra of E-field, $\mathrm{H}$ - field and $\mathrm{S} 21$ at $\mathrm{h}=100 \mathrm{~nm}$ and $\mathrm{a}=480 \mathrm{~nm}$. There is a sharp peak in the S21 spectrum, corresponding to the narrowband MR. 


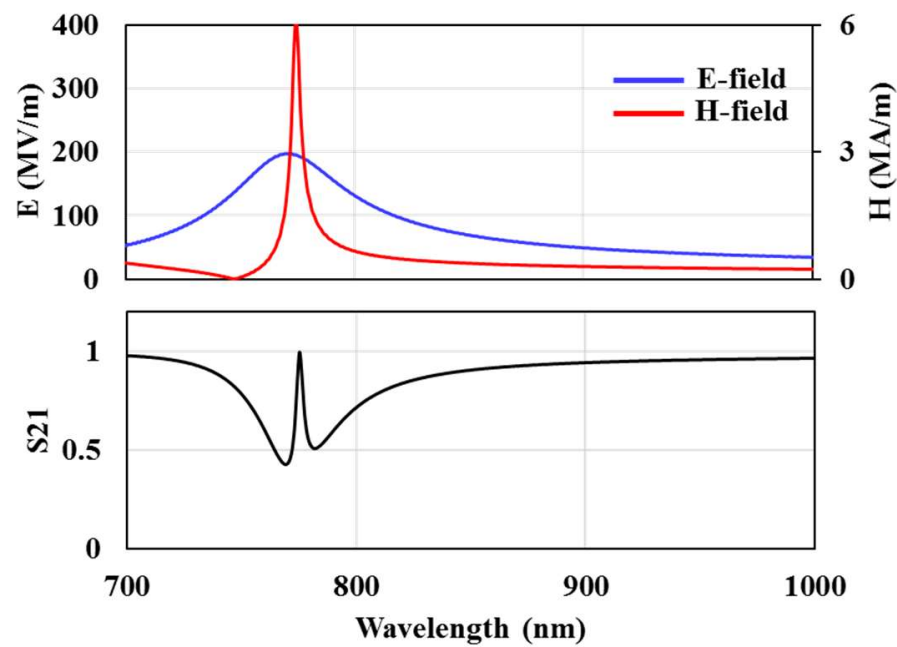

Figure 4.3 Spectra of a) E- and H- field intensity in the middle of silicon disk, and b) Transmission spectrum calculated by using the one cell model.

At $a=640 \mathrm{~nm}\left(3^{\text {rd }}\right.$ column in Figure 4.2), no effects related to coincidence of two resonances could be detected, and also no signs of wideband full transmission could be found. Thus, it becomes obvious that at increasing the lattice constant that has to make resonant processes in disks less dependent on interaction with neighbors, overlapping of electric and magnetic resonances in elements of metasurfaces should produce decreasing effect. It follows that coincidence of resonances cannot be considered as an effect typical for a single DR. Coincidence is impossible unless the structure of metasurface provides strong integration of resonant processes in array elements capable to cause such distortions of resonance modes, without which the coincidence of resonance frequencies could not be realized. By other words, the effects revealed in $[15,95]$ and in our work at $a=330 \mathrm{~nm}$ should not be considered as related to scattering of single particles at Kerker's conditions and should be analyzed, instead, by considering coupling between resonances in neighboring disks [8] and formation of Bloch's modes accounting for the structure of DR arrays [18].

Figure 4.4 shows the dependence of ER resonant frequency and wavelength on the lattice constant at $h=100 \mathrm{~nm}$. As seen in the figure, the ER frequency shifts down at increasing lattice constant, while the lengths of waves providing the resonance grow up, respectively. It can result from spreading the halos of resonance fields in more sparse arrays compared to squeezed and distorted halos in heavily packed arrays, in which DRs become incapable 
of confining longer wavelengths.

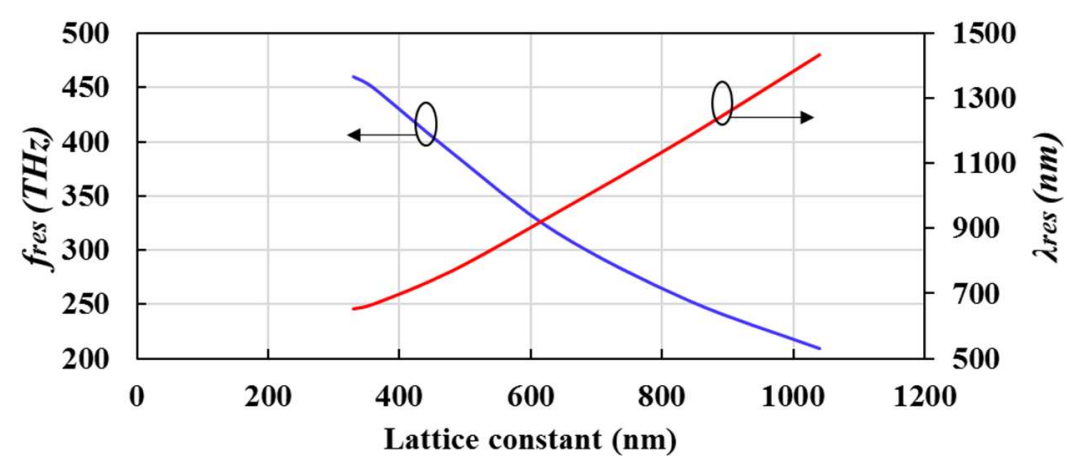

Figure 4.4 Dependence of ER resonance frequency on the lattice constant

\subsection{Correlation between EM responses of metasurfaces and dispersion phenomena in stacked DR arrays.}

To evaluate possible contribution of dispersion phenomena in responses of metasurfaces, we investigated the correlation between these responses and dispersion diagrams of DR array formed by stacking metasurfaces in the direction of wave vector (normal to the metasurfaces). Correspondingly, we compared S21 spectra simulated for one cell model with scattering spectra for five stacked metasurfaces, capable of representing infinite arrays [25]. Figure 4.5 presents spectral characteristics of array responses in four groups from A to D. Each group shows results for different thicknesses of disks, i.e. A, B, C and D for $h=140 \mathrm{~nm}, h=120 \mathrm{~nm}, h=100 \mathrm{~nm}$, and $h=80 \mathrm{~nm}$, respectively. There are three columns in each group which differ by array lattice constant $a$. Four rows in each group, respectively, illustrate S21 spectra for single cell model (first row), probe signal spectra for E- and Hfields at single cell simulations (second row), dispersion diagrams calculated for infinite arrays (third row), and S21 spectra for five planar arrays stacked in the direction of wave propagation (fourth row).

S21 spectra for the single cell model, especially in the group C for $a=330 \mathrm{~nm}$, are essentially different from those for five stacked cells. As it was mentioned previously, single cell model, often used at studying metamaterials, can represent properties of arrays adequately only when arrays are homogenized and all cells respond identically. The difference, often occurring between the responses of one cell and of an array, is usually 
assumed to be related to the influence of dispersion phenomena, characteristic for $\mathrm{PhC}$ structures. As can be seen from $3^{\text {rd }}$ and $4^{\text {th }}$ rows of each group, calculated dispersion diagrams agree well with the S21 spectra for the five cell model. We see that bandgaps and transmission branches in dispersion diagrams correlate well with the drops and the transmission frequency range in the S21 spectra. This correlation is less obvious in S21 spectra simulated for one cell model. However, the fact, that such correlation is still seen, let for considering the dispersion phenomena as involved in the formation of EM responses from metasurfaces.

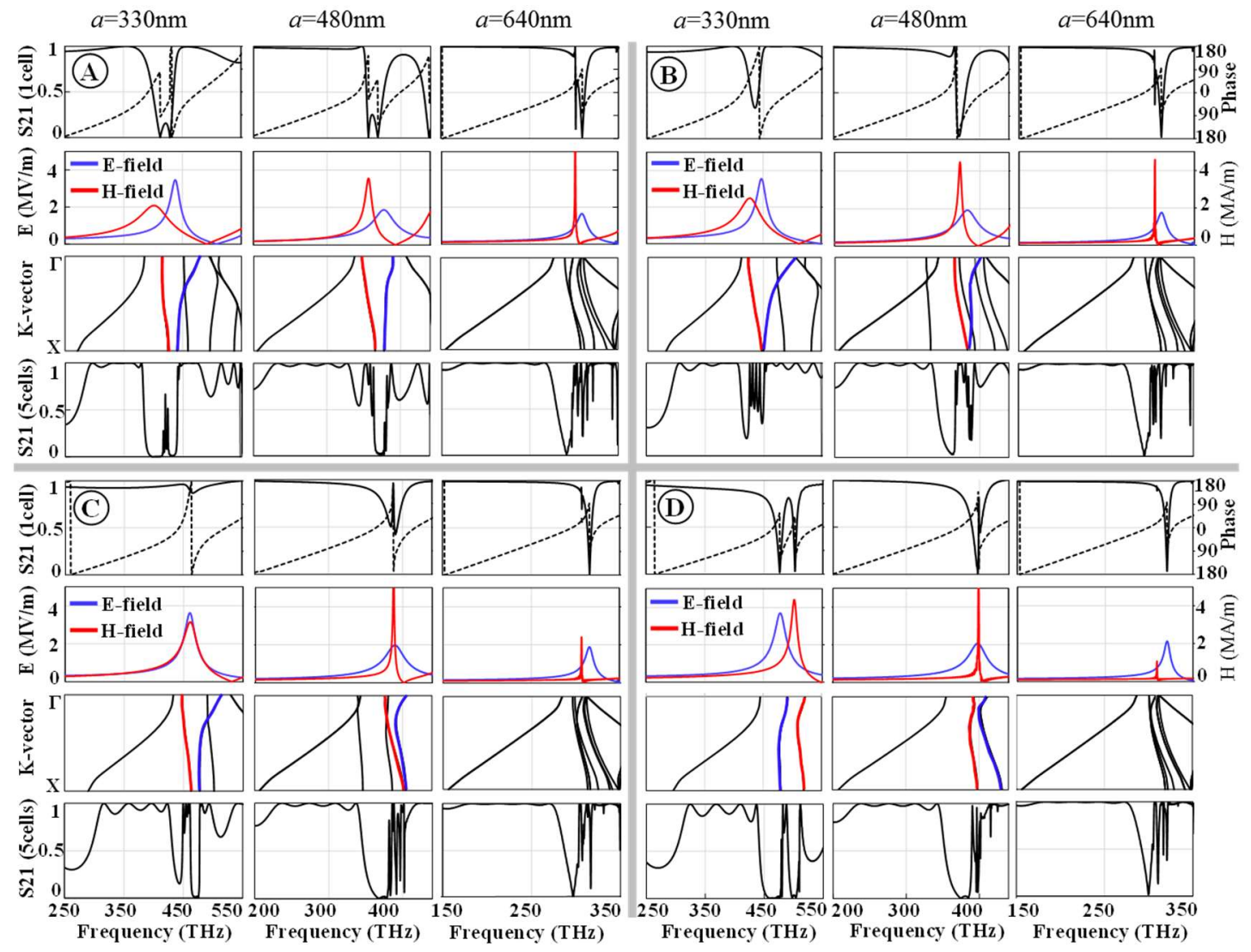

Figure 4.5 Four groups A-D present the results for four types of arrays, which differ by the disk thickness, in nm: A-140, B-120, C-100, and D-80. Columns in each group represent arrays with different lattice constants, in nm: 1 st column -310 , 2nd -480 , 3rd -640. Four rows in each group(from top to bottom) present, respectively, S21 spectra for one cell model, E- and H- field probe signal spectra, dispersion diagrams, and S21 spectra for five arrays stacked in the direction of wave propagation.

Analyzing the probe signal spectra presented in the $2^{\text {nd }}$ rows of all four data groups, we can see that peaks in probe signals, representing two resonances in first two columns of the 
each group, can be associated with two specific transmission branches in dispersion diagrams. We have marked these branches by the same colors as resonances, i.e. MR related branch with 'red', and ER related branch with 'blue'. We see that at lower lattice constants, MR related branches demonstrate slopes, characteristics for wave propagation in the media with positive refractive indices, while ER related branches, just opposite, acquire negative slopes and, therefore, represent negative refractive indices. Taking into account the data described in Chapter 3, it could be suggested that colored branches in dispersion diagrams support propagating resonance modes, i.e represent integrated responses of arrays with strongly coupled resonators. Figure 4.6 shows an example of field pattern for arrays of five stacked cells with $h=120 \mathrm{~nm}$ and $a=330 \mathrm{~nm}$. The field snapshots in the figure also show that at frequencies characteristic for MR related branch (red curve in respective dispersion diagram), we can see the propagation mode involving magnetic resonances in participating disks, while at frequencies characteristic for the ER related branch (blue line in diagram) propagation mode represents electric resonances in neighboring disks.
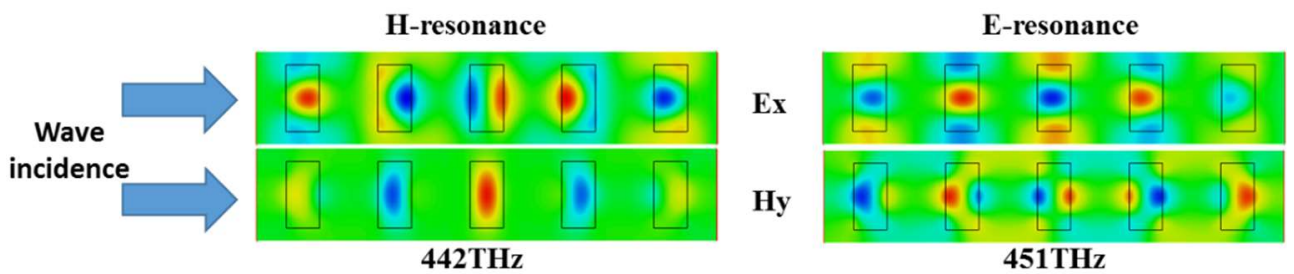

Figure 4.6 E- and $\mathrm{H}$ field pattern snapshots at a) frequency of MR related branch, and b) frequency of ER related branch in dispersion diagram

It can be also noticed in Figure 4.5 that at increasing the lattice constant, the Q-factor of resonances, especially of $\mathrm{MR}$, becomes higher, as it should be at decreasing the distortions of resonances and at their less integration in sparse arrays compared to that in dense arrays. To show the difference in resonance integration in sparse and dense arrays, Figure 4.7 compares the E- and H-field snapshots for disk array fragments with lattice constants of $330 \mathrm{~nm}$ and $640 \mathrm{~nm}$ at ER and MR frequencies in XY cross-sections. The thicknesses of disks are chosen to be $h=140 \mathrm{~nm}$ at the comparison. As seen in Figure $4.7 \mathrm{a}$, at ER for the case $a=330 \mathrm{~nm}$, strong E-fields confined in the resonators are observed and there are also 
E-fields squeezed in gaps along $\mathrm{x}$-direction between resonators. It is seen that interresonator E-fields in gaps in y-direction are largely enhanced opposite to that in gaps in $\mathrm{x}$ direction. At increasing the lattice constant to $a=640 \mathrm{~nm}$, both the confinement of electric dipoles and squeezing of field lines in the inter-resonator gaps in y-directions become decreased, while E-fields contributing to coupling along x-directions become enhanced. High E-field intensity in dense arrays shows more integration of ER response in these heavily packed arrays compared to that in sparse arrays. Magnetic dipoles in both arrays are mostly confined within resonators and they are less coupled than electric dipoles. Considering the field intensity in dense and sparse array, we can conclude, however, that most of the incident energy in dense arrays is confined between resonators in the form of E-field related energy, while in sparse arrays resonators are acting, rather, as individual particles and show stronger MR response. These observations confirms our previous statement that the ER and MR responses in dense arrays are less defined by single cell scattering than by the inter-resonator coupling in the array.

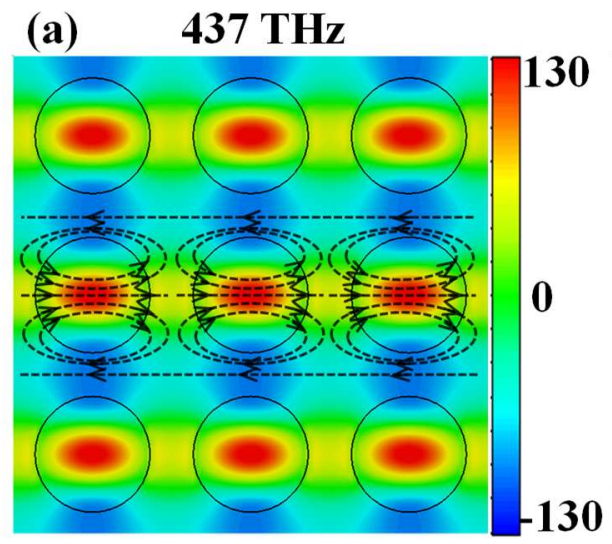

(b) $309 \mathrm{THz}$

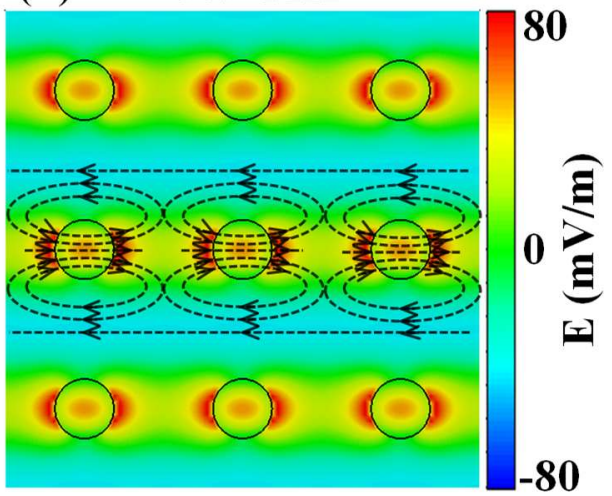

(c)

$402 \mathrm{THz}$

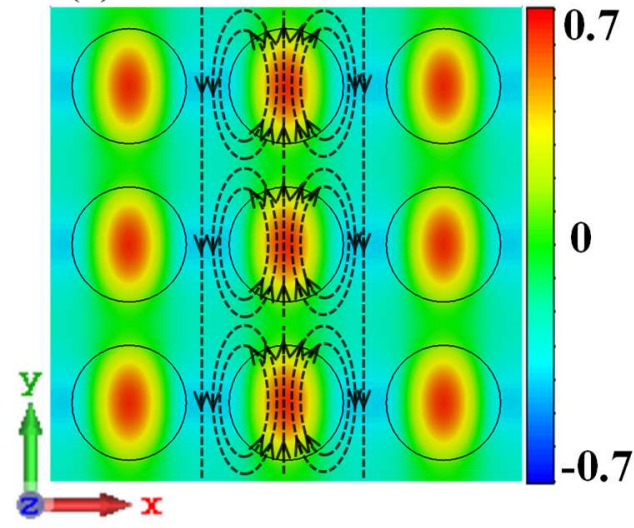

(d)

$301 \mathrm{THz}$

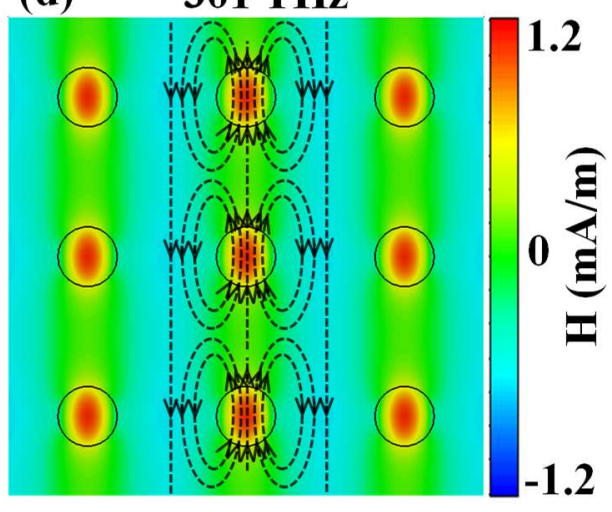


Fig. 4. (a, b) E-field and (c, d) H-field patterns in xy-cross-sections of 3x3 fragments of arrays with lattice constants of $330 \mathrm{~nm}$ (left column), and $640 \mathrm{~nm}$ (right column) at ERs and MRs observed, respectively, at (a) $437 \mathrm{THz}$, (b) 309 THz, and (c) $402 \mathrm{THz}$, (d) $301 \mathrm{THz}$. Disk radius - $120 \mathrm{~nm}$, disk thickness - $140 \mathrm{~nm}$.

\subsection{Conclusion}

Obtained in this chapter results do not support consideration of metasurfaces formed from dielectric resonators as arrays of independent particles. Instead, it is shown that resonators in arrays are integrated by coupled fields. In addition, these arrays appear to have a lot in common with $\mathrm{PhCs}$, in which array responses strongly depend on lattice parameters. Coincidence of MRs and ERs appears only in heavily packed arrays, so that transmission through arrays cannot be described by wave scattering from single particles at applying the concepts of Kerker's conditions. Investigated planar arrays cannot be considered as MMs, i.e. as homogenized media characterized solely by effective parameters. Analysis of array responses should account for their periodicity and dispersion properties. 


\section{Selected problems of implementing all-dielectric photonic crystals in the media of transformation optics based devices}

\subsection{Introduction}

This chapter describes my work on solving some problems, which make incorporating of photonic crystals (PhCs) in transformation media of EM devices extremely challenging. As it was mentioned in section 1.5, EM devices designed by using the transformation optics theory tend to require transformation media with constitutive parameters, which are anisotropic and often, in addition, have singular values, in particular, close to 0 , at least in one direction, to support superluminal phase velocity of passing waves. Therefore, one of the first tasks at incorporating PhCs in EM devices was to find PhCs capable of supporting superluminal wave propagation. In [122] and other earlier works of our team it was shown that close to zero index values could be expected in 2D PhCs of dielectric rods, if relative dielectric permittivity of the latter is higher than that in conventional PhCs. In [114] it was clarified, that superluminal wave propagation through arrays of dielectric resonators can be obtained at frequencies of the $2^{\text {nd }}$ transmission band in dispersion diagrams of respective $\mathrm{PhCs}$ near the upper edge of the bandgap between this band and fundamental band. In the next work, conducted with my participation [123], it was confirmed that the above mentioned $2^{\text {nd }}$ band is the product of splitting of fundamental band and of other transformations experienced by dispersion diagrams of DR arrays at an increase of rod permittivity.

Below in section 5.2 we describe changes in positioning of the $2^{\text {nd }}$ transmission band in dependence on the array parameters.

Next direction of our efforts became clear after it was decided to design PhCs-based transformation media from fragments of crystals with different lattice parameters. In particular, we developed a cylindrical cloak from the above fragments, which looked as ring-like structures, each composed of several circular arrays of infinite rods. Such design gave us an opportunity to approximate the requested by the TO dispersions of indices in 
the cloak medium by step functions. However, in [123] we took care about only radial dispersion of azimuthal index components, since it allowed for controlling superluminal wave propagation. Prescribed radial dispersion of radial index components was not provided in [123]. However, it did not deteriorate observation of the cloaking effect. Analysis of this unexpected result in the next paper of our team [103] has let us to realize the potential of self-collimation in cloaking (See section 5.3).

The decision to form the cloak from crystal fragments has demanded an investigation of the minimum quantity of circular arrays of dielectric particles, which we used to form crystal fragments. This quantity had to provide electromagnetic responses of fragments close to those from infinite arrays, so that we could design the cloak by using the results of simulations performed for infinite arrays. This minimal quantity of arrays was determined in the course of studies described in section 5.4 (first part of it).

Next question, which appeared in relation to fragmental cloak design, was if neighboring arrays of different fragments are able to affect responses of each other and thus, provide a kind of integration or averaging of the reaction of fragments to incident waves. The situation with integration is highlighted at the end of section 5.4.

The last section 5.5 of this chapter pays attention to additional opportunities of employing the self-collimation phenomena for hiding objects. In particular, we show the possibility to employ rod arrays with deformed cells to send waves around an elongated area, which can be used for hiding. Although proposed unidirectional cloak has fewer advantages compared to omnidirectional cloak, its design is easier to implement that promises future applications.

\subsection{PhC parameters providing superluminal wave propagation}

Considering employment of $\mathrm{PhC}$ structures for obtaining superluminal wave propagation, we have examined in this section 2D PhCs composed of low-loss dielectric rods with various parameters. Results presented in Figure 5.1 for PhCs with square lattices allow for determining the ranges of rod permittivity values and aspect ratios of rod radius $r$ to lattice constant $a$, at which superluminal propagation could be observed. 


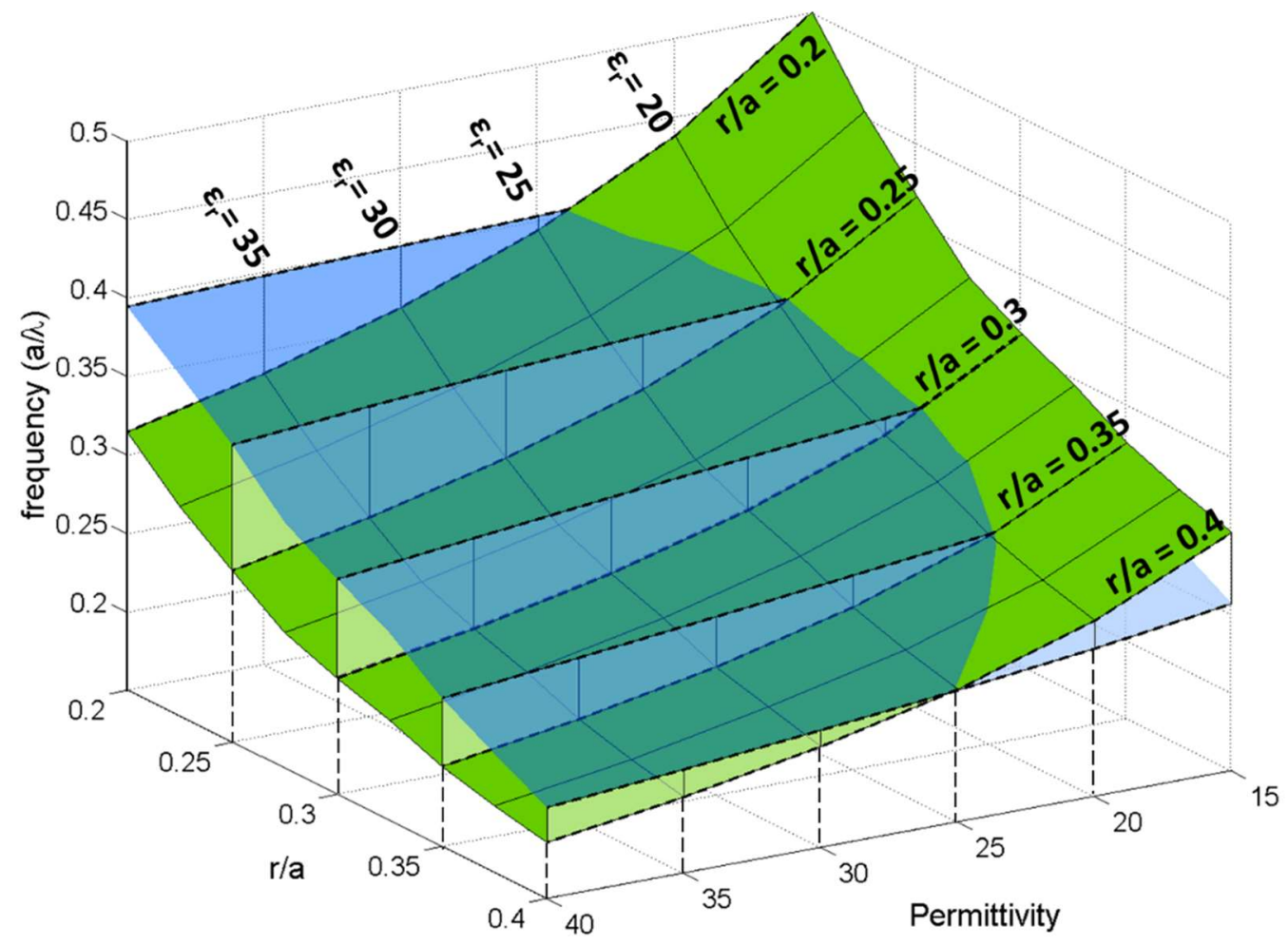

Figure 5.1 Data extracted from dispersion diagrams, calculated for PhCs with square lattices in wide range of rod permittivity values and aspect ratios. Green surface shows locations of the lower edges of $2^{\text {nd }}$ transmission bands at the energy/frequency scale, while blue surface characterizes the locations of Bragg resonance. Crossing of two surfaces demonstrates parameters, at which sinking of the original $2^{\text {nd }}$ transmission bands into the fundamental band causes an opening in this band of a new bandgap and formation above this bandgap of a novel transmission band with positive refraction indices ranging from zero values at the lower edge of the band up to values exceeding 1 at the upper band edge [123]. The gap between two surfaces seen when blue surface is located above green one, represents a novel $2^{\text {nd }}$ transmission band.

This figure shows at which values of rod permittivity and aspect ratios we can observe appearance of the new $2^{\text {nd }}$ transmission band and how one of two parameters could be changed if another one is fixed at some value. As seen in Figure 5.1, the width of the $2^{\text {nd }}$ band at given $\varepsilon_{\mathrm{r}}, \mathrm{r}$ and $a$, which is defined by the gap between two surfaces, can be significantly changed at varying the parameters of PhCs. For example, at $\varepsilon_{r}=35$, changing the $r / a$ from 0.4 to 0.2 can be used to extend the width of the $2^{\text {nd }}$ band up to several times. It can decrease the steepness of the dependence of index values on frequency in the range with superluminal wave propagation and, thus, affect the bandwidth of the cloaking effect. The curve marking the crossing of two surfaces allows for determining the lowest 
permittivity values, at which wave propagation is still controlled by positive indices of refraction. For example, at $\mathrm{r} / a=0.2$, switching to positive indices becomes possible at the relative permittivity over 24 .

\subsection{Bending the wave paths in cylindrical cloak composed of rod arrays}

Prescriptions for refractive indices in the medium of TO-based cloak, which resulted from transforming an infinitely long cylindrical region with $R \leq R_{\text {out }}$ to a cylindrical shell $R_{\text {in }} \leq$ $R^{\prime} \leq R_{\text {out }}$, could be represented by next expressions [123]:

$$
\begin{gathered}
n_{R}=1 /\left(1-2 R_{\text {in }} / R_{\text {out }}+\frac{2 R_{\text {in }} R}{R_{\text {out }}^{2}}\right) \\
n_{\theta}=\left(R / R^{\prime}\right)
\end{gathered}
$$

where $R_{\text {in }}$ and $R_{\text {out }}$ are the inner and the outer radii of the shell cross-section. However in [123] we took into consideration only the azimuthal components responsible for realizing wave propagation with superluminal phase velocity, and employed photonic crystal fragments with square lattices, which could provide only identical radial dispersion for azimuthal and radial components of refractive indices. Figure 5.2a shows simulated wave pattern in a cloak media with identical radial dispersion of both azimuthal and radial index components. It is clearly seen that such media should not provide bending of wave paths around the target and cause cloaking effect.

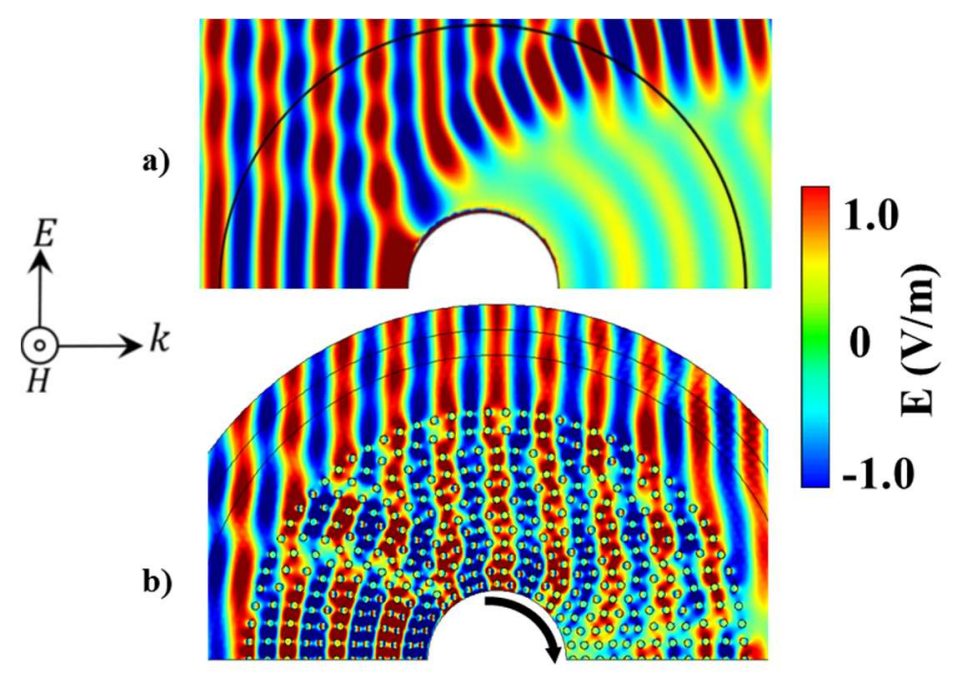

Figure 5.2 a) the simulated wave pattern for the model of cloak media with identical azimuthal and radial component of refractive indices, b) the wave pattern observed in the cloak built of square-latticed fragments 
of PhC: it is seen that waves pass around the target

Figure 5.2b shows a wave pattern taken from [123] for a medium composed of $\mathrm{PhC}$ fragments with square lattice, which as it has been mentioned above was capable to provide only identical radial dispersion of azimuthal and radial components. In the figure, we observe a wave turning around the object and unexpected cloaking effect which has to be related, obviously, to other physical mechanism, instead of to refraction. Such mechanism could be provided by well-known in PhCs phenomenon of self-collimation. According to [124], this phenomenon provides wave propagation along crystallographic axes of PhCs, even if axes are bent. Figure 5.3, presents another wave pattern observed in the PhCs based cloak [123], which clearly demonstrates self-guided waves along the circumference in the outer PhC layers of the cloak, while beyond the cloak, a shadow similar to that presented in Figure 5.2a can be clearly seen.

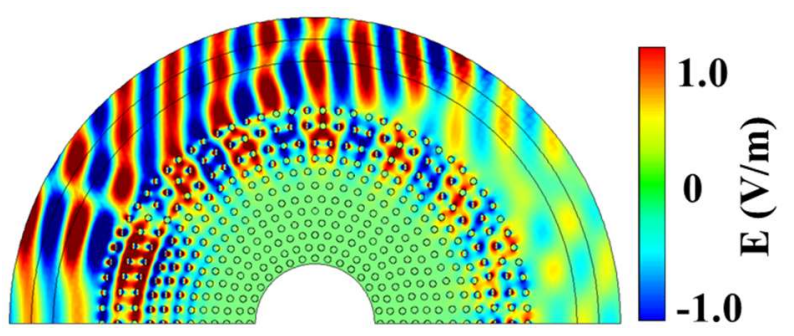

Figure 5.3 wave patterns observed in the PhCs based cloak in [123] that shows outer layer is guiding the wave along the circumference.

Considering the above observations, we have concluded that employing $\mathrm{PhCs}$ in the cloak medium allows for using self-collimation in $\mathrm{PhCs}$ to realize TO-requested functionalities of the cloak medium, even if prescriptions for material properties could not be fully realized. As mentioned above, self-collimation is the phenomenon of guiding waves by photonic crystal along one of its crystallographic directions normal to EFCs [125]. Self-collimation (also known as auto-collimation or self-guiding) allows an electromagnetic wave to propagate in $\mathrm{PhC}$ structures without any significant broadening or change in the beam profile, and also without relying on a bandgap or engineered defects [126]. This phenomenon was employed for beam bending in [124, 125], in optical devices such as splitters in [127] and in analogs to digital converters in [128], as well as for routing optical signals $[129,130]$. However, bending of the wave paths in TO controlled devices using the 
self-collimation has not been studied so far and was never used for realizing the effect of invisibility at employing cloaking devices. The only known attempt to employ the selfcollimation for concealing was reported in [131] where authors proposed to use it for controlling the reflections from some object (in so-called reflection cloak). Therefore, after realizing the possibility to employ the self-collimation for wave paths bending within the cylindrical TO-governed cloak, we decided to investigate an opportunity of building up the unidirectional transmission invisibility cloak based on the self-collimation in $\mathrm{PhCs}$ (section $5.5)$.

\subsection{Optimal structure of PhC fragments and clarifying the problem of EM interaction between fragments}

As it was mentioned in section 5.1, we composed the cloaking shell from concentric arrays of dielectric rods with the same lattice constants within each of $\mathrm{PhC}$ fragments, but with essentially different lattice constants of arrays used in various fragments. Such design transformed square unit cells into trapezoidal ones that could affect their properties. However, more serious problem was seen in the need to use minimal possible number of arrays in each fragment, to prevent the thickness of the cloak from impractical increase, while, on the other hand, each of $\mathrm{PhC}$ fragments had to incorporate such number of circular arrays, which would provide mimicking infinite crystals to conserve an opportunity for calculating refractive indices of fragments. To find solution to these contradictory demands, we simulated field probe signals and wave patterns in several array sets with various number of linear arrays in each set (Figure 5.4). We chose for simulation arrays with rod permittivity of 35 , rod radius of $1.5 \mathrm{~mm}$, and lattice constants of $5.55 \mathrm{~mm}$. From the results in section 5.2 it followed that in infinite arrays with such parameters, the upper edge of bandgap (onset of $2^{\text {nd }}$ transmission band) could be observed near $14 \mathrm{GHz}$. The spectra of signals from field probes placed in arrays far from the source present, in fact, the data comparable to S21 spectra. As seen in Figure 5.4b, PhC fragments containing 3 or more parallel rod arrays demonstrate similar responses in the spectra of probe signals, showing, in particular, a sharp rise around $14 \mathrm{GHz}$. Since the results for fragments composed of 2 linear arrays appeared different from the results for fragments composed of 3 or more linear 
arrays, it could be concluded that PhCs fragments composed of 2 arrays are incapable of mimicking the responses of infinite $\mathrm{PhCs}$ with similar parameters. Therefore, we excluded fragments composed of 2 linear arrays from further consideration and did not present probe signal spectra observed in these fragments in Figure 5.4b. Snap-shots of field distributions in cross-sections of fragments composed of 3 and more linear rod arrays are presented in Fig. 5.4c. These snap-shots allowed for identifying 'odd' and 'even' transmission modes characteristic for infinite rod arrays. Even modes were the subject of our special interest, since their formation confirmed superluminal wave propagation and thus, an opportunity to use respective fragments in the cloak medium. As seen in Figures 5.4c, $\mathrm{PhC}$ fragments composed of 3 linear arrays (triplets) demonstrate superluminal wave propagation, similar to that observed in fragments composed of 4 and more linear arrays. This result allowed for choosing array triplets as the basic units at forming $\mathrm{PhC}$ fragments, which provide $\mathrm{EM}$ responses close to those of infinite arrays at minimal number of constituting arrays.

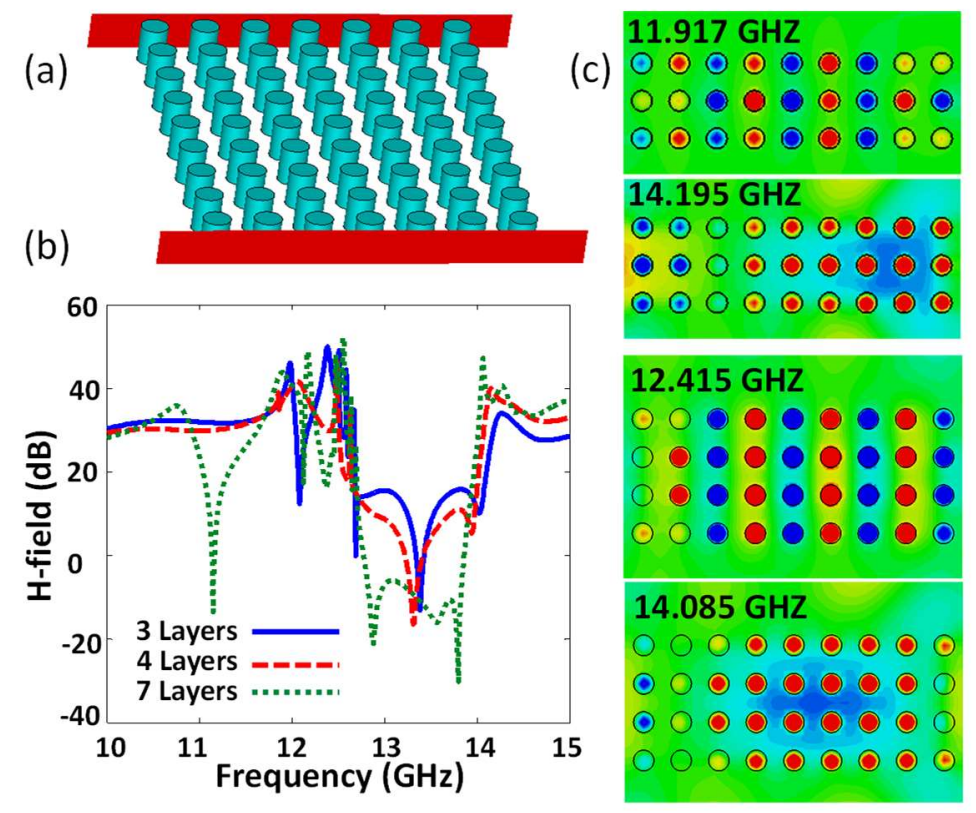

Figure 5.4 (a) Simulation model, (b) probe signal spectra, and (c) snap-shots of odd and even mode formation observed near the bandgap edges for array sets composed of 3, and 4 linear arrays in parallel with the lattice constant of 5.55 mm. (Fig. 8 in ref. [123], (c) 2016 IOP Publishing. Reproduced with permission. All rights reserved. See Appendix B for documentation of permission to republish this material.) 
The next problem to be addressed was the choice of separation distance between triplets to understand, if neighboring fragments of triplets can affect responses of each other. In fact, interaction between closely placed array triplets could produce dual influence on their responses. If close presence of other triplets deteriorated superluminal transmission, it would be an undesirable effect. On the other hand, we could expect positive effects if neighboring triplets could provide a kind of averaging of the reaction to incident waves, which could lead to smoother changes of indices in the fragmented cloak. To investigate the effects of neighboring arrays on each other, we simulated probe signal spectra and field patterns for structures composed from 3 close packed array triplets with different lattice parameters: $5.55 \mathrm{~mm}, 6.0 \mathrm{~mm}$, and $6.52 \mathrm{~mm}$ (Figure 5.5). The distance between neighboring arrays of employed triplets was taken equal to the lattice constant of the sparser triplet. For example, we took the distance of $6.52 \mathrm{~mm}$ between neighboring arrays of triplets with lattice constants of $6.0 \mathrm{~mm}$ and $6.52 \mathrm{~mm}$. As seen in Fig.5.5, the probe signal spectra of employed triplets have demonstrated characteristic peaks at the upper edges of the bandgap: at $13.65 \mathrm{GHz}$ for array with $a=6.52 \mathrm{~mm}$, at $13.85 \mathrm{GHz}$ for $a=6.00 \mathrm{~mm}$, and at $14 \mathrm{GHz}$ for $a=5.55 \mathrm{~mm}$ (shown by dashed vertical lines in Figure 5.5). As seen in the snapshot at the frequency of $13.65 \mathrm{GHz}$, when the array triplet with lattice constant of $6.52 \mathrm{~mm}$ supports superluminal wave propagation, similar propagation can also be seen in nearest array of the neighboring triplet ( $a=6.00 \mathrm{~mm}$ ), despite such propagation could be expected to occur at higher frequency $13.85 \mathrm{GHz}$. At the frequency of $13.85 \mathrm{GHz}$, the superluminal wave propagation in the central triplet $(a=6.00 \mathrm{~mm})$ also involves two linear arrays from the triplet with smaller lattice constant $(a=5.55 \mathrm{~mm})$, although this triplet with smaller lattice constant is expected to show superluminal wave propagation at $14 \mathrm{GHz}$. The obtained results show that neighboring triplets do not deteriorate the superluminal response of each other. They, instead, involve each other in supporting superluminal wave propagation and provide averaging of the reaction of triplets to incident waves. Thus, the revealed integration of triplet responses was expected to make smoother the steps of index changes at moving from one $\mathrm{PhC}$ fragment to another along the radius of the cloak. It should improve approximation of index dispersion in the cloak by step function. 

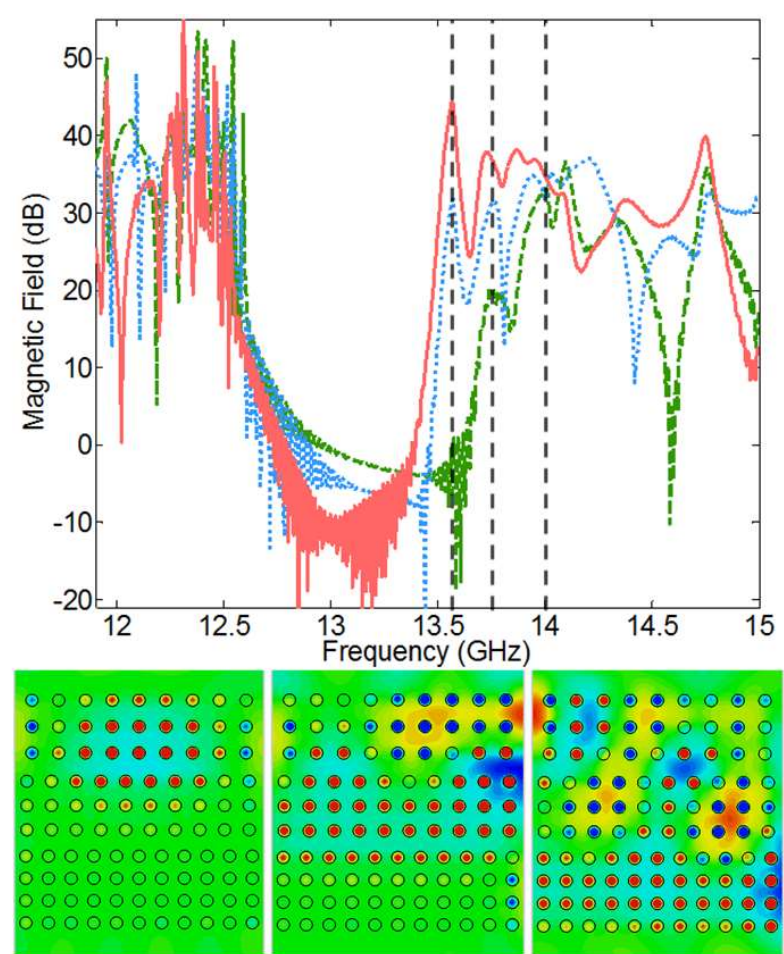

$13.564 \mathrm{GHz}$

13.7 $\mathrm{GHz}$

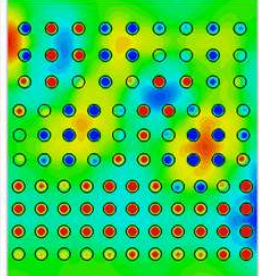

$14.01 \mathrm{GHz}$

Figure 5.5 Upper figure: signal spectra from probes located in centers of one array quartet with lattice constant $5.55 \mathrm{~mm}$ (dashed curve) and two array triplets with constants $6.0 \mathrm{~mm}$; and $6.52 \mathrm{~mm}$ (dot and solid curves). Vertical dashed lines show positions of peaks observed at superluminal transmission in respective array sets. Lower row: snapshots of field patterns in array cross-sections demonstrating areas of superluminal response and involvement of neighboring triplets. (Fig. 10 in ref. [123], (C) 2016 IOP Publishing. Reproduced with permission. All rights reserved. See Appendix B for documentation of permission to republish this material.)

\subsection{Additional options for employing the self-collimation in cloaking devices}

In the presented below work, we demonstrate a possibility to realize the phenomenon of self-collimation in PhCs with specifically deformed lattice cells that allows for obtaining the cloaking effect by simpler means using unidirectional cloaking structure. The rod radius was chosen to be $r=1.5 \mathrm{~mm}$, and the dielectric permittivity $-\varepsilon_{r}=35$. We have taken the lattice constant equal to $a=5.85 \mathrm{~mm}$, which was employed in [123]. The extracted dispersion diagram ensured that the rod array with chosen parameters should be capable to respond with positive refractive indices starting from zero, and thus should support the wave propagation with superluminal phase velocities. Two symmetric in structure rod 
arrays with curvilinear crystallographic axis in $\mathrm{X}$ direction were used to hide the elongated metal object by guiding the incident waves around the object without any reflections, thus making the object invisible. The field pattern at passing the waves through the new device at $14.2 \mathrm{GHz}$ is shown in Figure 5.6a. It is seen that the front of incident wave becomes split and after coming the wave flow close to the hidden object, the waves begin to move along two curvilinear paths around the object. Therefore, our numerical experiments on rod arrays with properly deformed crystallographic axes have confirmed the possibility to use such arrays for directing waves along proper axes, no matter if they were curved. The structure, realized in the course of our experiments, could be considered as unidirectional transmission cloak.

a)
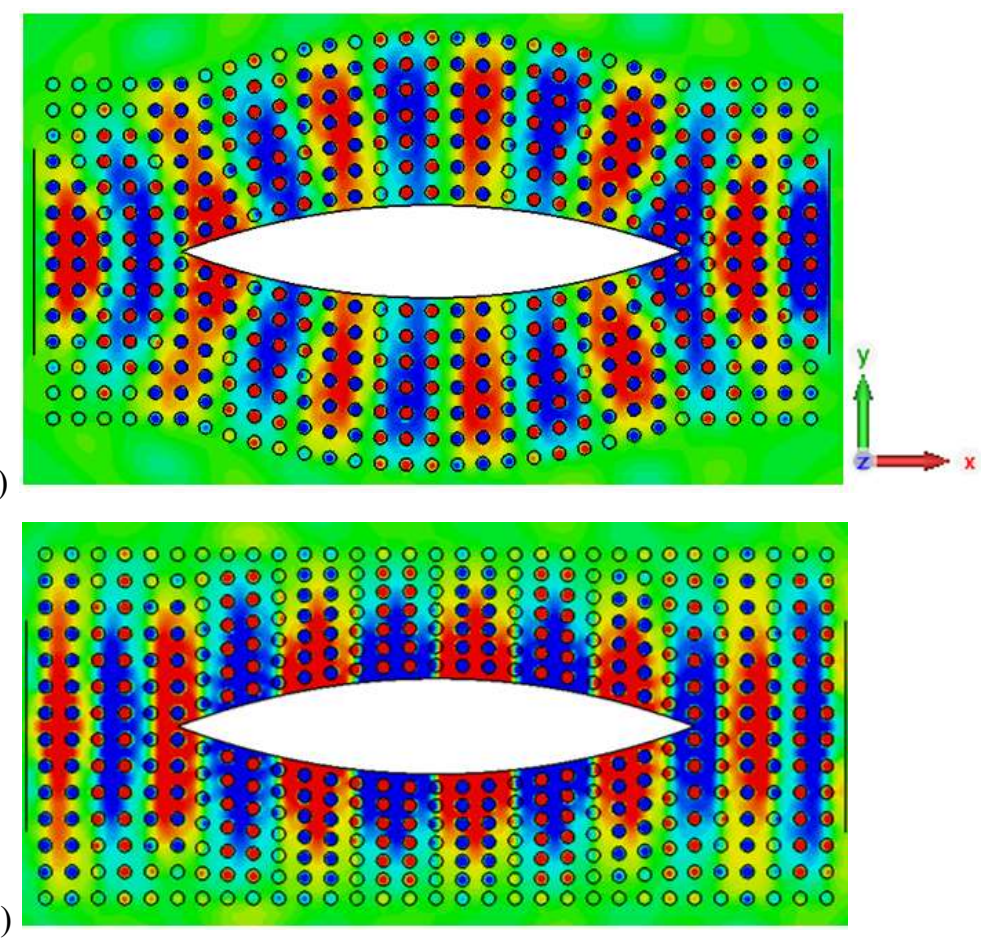

Figure 5.6 Self-guiding of waves a) through the deformed array along curved trajectories around the object and b) in PhC waveguide with fixed cross-section provided by squeezing Y-oriented sides of the lattice cells near the object at $\mathrm{f}=14.2 \mathrm{GHz}$. (Fig. 2 and Fig. 3 in ref. [132], (C) 2016 IEEE. Reproduced with permission. All rights reserved. See Appendix $\mathrm{C}$ for documentation of permission to republish this material.)

To mitigate the drawback of the proposed structure - changing the $\mathrm{Y}$ size along the device length - we have proposed to deform the rod array additionally, so that its cross-section would be kept constant along the entire length (Figure 5.6b). Thus, we squeezed the cloak 
in $\mathrm{Y}$ direction near the object, while the dimension along $\mathrm{X}$ axis was remained unchanged. For the squeezed medium, the lattice constant along Y direction is decreased to $4.08 \mathrm{~mm}$. As seen from Figure 5.6b, squeezing did not deteriorate self-guiding of waves along crystallographic direction, which is not so much different from $\mathrm{X}$ one. It is also seen in Figure $5.6 \mathrm{~b}$ that in the squeezed medium, the wavelengths and thus the phase velocities of waves are up to 2 times bigger than those at the waveguide/device ends. Respective speeding of waves up is usually required in TO-based cloaks for restoring the wave front beyond the cloak. To ensure that observed speeding of waves in our case is defined by changes in lattice structure of arrays, we have extracted for comparison, the spectra of refractive indices for PhCs with both square lattice $5.85 \mathrm{~mm} \times 5.85 \mathrm{~mm}$ used at the waveguide ends, and rectangular lattice $5.85 \mathrm{~mm} \times 4.08 \mathrm{~mm}$ used at building up rod arrays around the hidden object in Fig.5.8b.

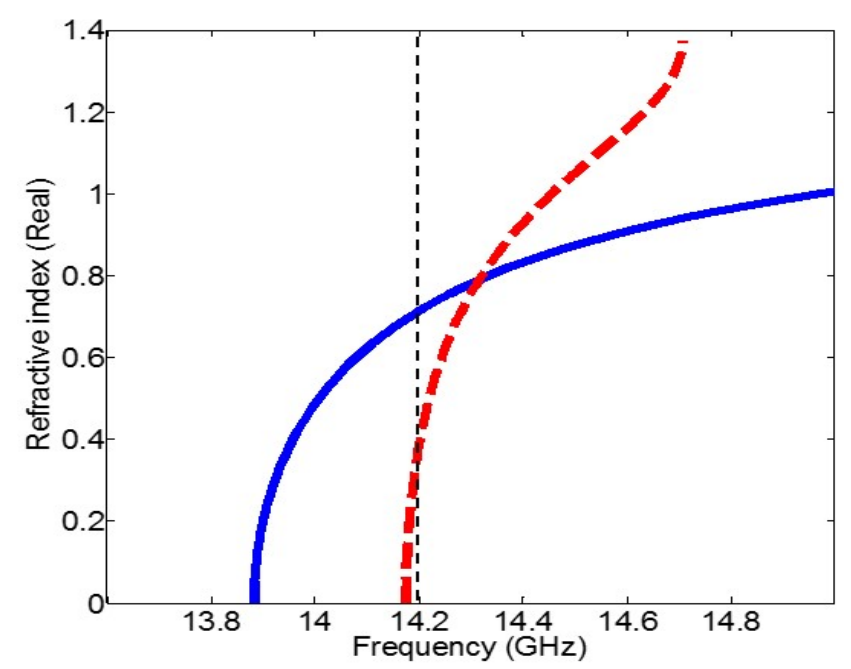

Figure 5.7 Frequency dependencies of refractive indices for X-directed wave propagation in the square lattice $5.85 \mathrm{~mm} \times 5.85 \mathrm{~mm}$ (blue/solid) and in the rectangular lattice $5.85 \mathrm{~m} \times 4.08 \mathrm{~mm}$ (red/dashed). (Fig. 4 in ref. [132], (C) 2016 IEEE. Reproduced with permission. All rights reserved. See Appendix C for documentation of permission to republish this material.)

As seen from Figure 5.7, at 14.2 GHz, the square lattice had much higher index in X direction than that of rectangular lattice, although dimension of both lattices in this direction were identical. It follows that the former lattice supports phase velocities more close to those in free space, while the latter lattice demonstrates superluminal wave propagation. It could be concluded that variable deformation of $\mathrm{PhC}$ lattice cells could be 
employed for controlling the phase velocity of waves in electromagnetic devices. However, in future works, refractive indices of cells in the cloak medium still have to be more precisely adjusted for providing the expected phase velocities.

\subsection{Conclusion}

In this chapter, we addressed several challenges, met at incorporating dielectric $\mathrm{PhC}$ in TObased devices. We first determined the changes in positioning and bandwidth of the $2^{\text {nd }}$ transmission band in dependence on the array parameters. We graphically showed, at which aspect ratio and rod permittivity rod arrays can exhibit positive indices starting from 0 , and can support superluminal phase velocity with wider bandwidth. Then we investigated responses of $\mathrm{PhC}$ fragments composed of various numbers of parallel linear arrays and have shown that $\mathrm{PhC}$ fragments, used in the cloak design, should incorporate not less than 3 parallel rod arrays to mimic responses characteristic for infinite $\mathrm{PhCs}$ with the same parameters. It was also revealed that neighboring array triplets in the cloak design could affect the EM responses of each other. However, at some distance between triplets, their interactions do not deteriorate superluminal wave propagation in $\mathrm{PhC}$ fragments, and, instead, involving neighboring triplets in joint responses appears useful for smoothing steps in radial index dispersion, caused by fragmenting the cloak media, that can improve the functionality of the cloak. We have also found that in the TO-based cloak composed of PhC fragments with square lattices, it is possible to realize desired wave-guiding controlled by the phenomenon of self-collimation, which provides bending of wave paths around the target, no matter if the TO prescribed radial dispersion of radial index components is provided or not. We have also demonstrated that self-collimated wave propagation along curved trajectories is possible at the superluminal phase velocity of waves and at squeezing the lattice cells of PhCs along the direction normal to the direction of wave propagation. This phenomenon can be used, when realizing conditions requested by the TO is impossible or too complicated. 


\section{Summary and future works}

\subsection{Summary}

In Chapter 1, we have discussed new role of dielectric materials in EM devices and advances in applications of dielectrics, which occurred due to development of new artificial materials, such as photonic crystals and metamaterials. These materials use periodic structures formed from macroscopic "atoms", which could be represented by either dielectric particles or dielectric resonators. Analysis of literature devoted to developing dielectric-based media allowed us to formulate a row of problems, solving of which was necessary for advancing applications of these media in EM devices.

In Chapter 2 we have described approaches to developing and fabricating novel RF probes for UHF MRI systems. The designs of these probes were inspired by microstrip patch antennas, however, we employed patch structures as resonators, radiation from which was undesirable. We used different options to control magnetic field uniformity above the patches in new probes, in particular, we used nonuniform multi-dielectric dielectric substrates engineered by proper embedding high permittivity pellets inside low permittivity hosts, and by modifying the shape of probes using profiled high permittivity substrates. We have shown that single patch structures could be successfully used as surface probes, while two vis-à-vis placed patches could act as volume probes, if fed with $180^{\circ}$ phase difference. The extracted images of B1 fields at MRI experiments with fabricated RF probes confirmed a possibility to create very uniform magnetic field distribution within the tested samples. We also compared Q-factors of the proposed volume patch probes with those of birdcage coils, and have shown that Q-factors of our probes were essentially more stable than Qfactors of birdcage coils, when probes were loaded with lossy samples. This property can be considered as a very promising advantage of patch probes over standard coils.

In Chapter 3 we have presented the results of our studies of negative refraction in alldielectric MMs composed of elongated dielectric rods. To define the factors leading to negative refraction we compared electromagnetic responses from arrays of such rods with different lattice parameters. In particular, we calculated the spectra of Mie resonances in single rods, extracted spectra of refractive indices from scattering parameter spectra of 
arrays, and compared the obtained data with dispersion diagrams. We also simulated field patterns in cross-sections of arrays at different frequencies. Our studies have shown that the phenomenon of negative refraction in DR arrays has no relation to overlapping of Mie resonances in single resonators, as it was conventionally supposed. Instead, appearance of negative refraction at some values of lattice constants was found to correlate with appearance of transmission branches of specific shape (with "negative' slope) in dispersion diagrams of arrays. It means that negative refraction often observed in MMs composed of dielectric rods cannot be considered as characteristic for conventional MMs phenomenon defined by double negativity of effective parameters. The real nature of this phenomena is similar to that in photonic crystals and is defined by dispersion properties of arrays owing to array periodicity.

In Chapter 4, we described investigations undertaken to realize the nature of wideband full transmission of incident light through dielectric meta-surfaces, represented by planar periodic arrays of silicon nano-disks. In literature this phenomenon has been considered as a confirmation of unidirectional front scattering of light by nano-disks due to realizing Kerker's scattering condition at coincidence of electric and magnetic resonances in disks. Such interpretation caused doubts, since Kerker's conditions were formulated for Mie resonances in single sphere. To clarify the observed phenomena, we studied the effects of array lattice parameters on their EM responses. It was found that increase of lattice parameters affected spectral positions of resonances in arrays and was capable of preventing two resonances in disks from coincidence and even of suppressing magnetic resonance completely. The obtained results contradict conventional views on metasurfaces, composed of DRs, as on assemblies of independent particles. Instead, we have shown that resonators in arrays are integrated by coupled fields so that their properties have a lot more in common with $\mathrm{PhCs}$, that with MMs. The wideband full transmission through such arrays appeared to be a result of mutual annihilation of electric and magnetic resonances and of defined by such annihilation deep drop in reflection losses.

Chapter 5 described our contribution to the solution of problems met at incorporating photonic crystals into the media of transformation optics - based devices, in particular, of invisibility cloaks. We have started from defining the ranges of array parameters - such as 
rod permittivity, rod radius, and the lattice constant, at which transmission through arrays could be characterized by close to zero values of positive indices, which are requested for wave propagation with superluminal phase velocities. It was confirmed that to realize such propagation in microwave arrays, they should be composed of rods with relative permittivity higher than 20 .

Then, we have contributed in developing the design of a cloak based on photonic crystals. It was found that the medium of the cloak could be formed from layers of different sizes, each of which integrated several concentric arrays of dielectric particles and, thus, represented the fragment of bent photonic crystal with specific lattice constants. To realize prescribed by the TO radial dispersion of index values within the cloak, fragments located closer to inner boundary of the cloak had to have smaller lattice constants, while fragments located close to outer boundary of the cloak - bigger lattice constants. We have shown that each of PhCs fragments should contain not less than three concentric array of dielectric particles with the same lattice constants to provide acceptable correspondence of refractive indices of these fragments to theoretical values characteristic for infinite array. Then the cloak could be formed by using 4 or 5 array triplets placed along the radius of the shell. Since the lattice parameters of the array triplets in $\mathrm{PhCs}$ fragments were chosen to provide the TO prescribed dispersion for only azimuthal index components, we could not expect bending of wave paths within the cloak. However, the designed cloak has demonstrated such bending and, therefore, the cloaking effect. Analysis of this phenomenon has shown that bending of wave paths was provided by self-collimation of passing waves within fragments of bent PhCs. Further investigations has allowed to ensure that self-collimation can be used instead of TO prescribed radial dispersion of radial index components for controlling superluminal movement of waves along curvilinear trajectories in

transformation media, including the cases when lattice cells are squeezed along the direction normal to the wave incidence.

\subsection{Future works}

The results obtained in these studies have inspired some possible future directions of work. 
As we mentioned in Chapter 2, the value of Q-factor of miniaturized patch probes appeared too low compared to that of birdcage coil. This happened, most probably, due to radiation losses of the probe. To increase the Q-factor, it is possible in future to use higher permittivity substrate such as RT3010 as the host instead of FR-4. At high permittivity of the substrate material, the probe radiation should be decreased and the higher Q-factor should be obtained.

Next, the uniformity of magnetic field above the patch in new probes was investigated only along $\mathrm{X}$ - and $\mathrm{Y}$ - directions in Chapter 2. It would be desirable to increase also the uniformity of B1 field along Z- direction, and for this purpose, tapered patch could be used. However, employing tapered patch may affect the uniformity along X- direction and, therefore, should be employed with care.

Further, the simulation results obtained for the cloak design proposed in Chapter 4 can be verified in future at prototyping the cloaking device. Moreover, since in the current work, only azimuthal component of the cloak was investigated, later, by applying rectangular lattice parameters, the prescribed dispersion for both radial and azimuthal component could be provided when the self-collimation is not effective. Controlling the contribution of the self-collimation in bending the wave paths could also become a subject of future work, to make possible designing the cloak based on self-collimation effects. 


\section{References}

[1] C. A. Balanis, Advanced engineering electromagnetics. John Wiley \& Sons, 1999.

[2] D. K. Cheng, Field and wave electromagnetics. Pearson Education India, 1989.

[3] A. G. Webb, "Dielectric materials in magnetic resonance," Concepts in Magnetic Resonance Part A, vol. 38A, no. 4, pp. 148-184, 2011.

[4] S. John, "Strong localization of photons in certain disordered dielectric superlattices," Physical Review Letters, vol. 58, no. 23, pp. 2486-2489, 1987.

[5] E. Yablonovitch, "Inhibited Spontaneous Emission in Solid-State Physics and Electronics," Physical Review Letters, vol. 58, no. 20, pp. 2059-2062, 1987.

[6] E. Yablonovitch, "Photonic band-gap structures," Journal of the Optical Society of America B, vol. 10, no. 2, pp. 283-295, 1993.

[7] J. B. Pendry, A. J. Holden, D. J. Robbins, and W. J. Stewart, "Magnetism from conductors and enhanced nonlinear phenomena," IEEE Transactions on Microwave Theory and Techniques, vol. 47, no. 11, pp. 2075-2084, 1999.

[8] Fang Chen, X. Wang, G. B. Semouchkin, and E. Semouchkina, "Effects of inductive waves on multiband below-cut-off transmission in waveguides loaded with dielectric metamaterials," AIP Advances, vol. 4, no. 10, p. 107129, 2014.

[9] E. A. Semouchkina, G. B. Semouchkin, M. Lanagan, and C. A. Randall, "FDTD study of resonance Processes in metamaterials," IEEE Transactions on Microwave Theory and Techniques, vol. 53, no. 4, pp. 1477-1487, 2005.

[10] V. M. Shalaev et al., "Negative index of refraction in optical metamaterials," Optics Letters, vol. 30, no. 24, pp. 3356-3358, 2005.

[11] G. Anthony and E. G. V., "Experimental verification of backward-wave radiation from a negative refractive index metamaterial," Journal of Applied Physics, vol. 92, no. 10, pp. 5930-5935, 2002.

[12]E. Cubukcu, K. Aydin, E. Ozbay, S. Foteinopoulou, and C. M. Soukoulis, "Negative refraction by photonic crystals," Nature, vol. 423, p. 604, 2003.

[13] S. Foteinopoulou and C. M. Soukoulis, "Negative refraction and left-handed behavior in twodimensional photonic crystals," Physical Review B, vol. 67, no. 23, p. 235107, 2003.

[14] Y. H. Fu, A. I. Kuznetsov, A. E. Miroshnichenko, Y. F. Yu, and B. Luk'yanchuk, "Directional visible light scattering by silicon nanoparticles," Nature Communications, Article vol. 4, p. 1527, 2013.

[15]I. Staude et al., "Tailoring Directional Scattering through Magnetic and Electric Resonances in Subwavelength Silicon Nanodisks," ACS Nano, vol. 7, no. 9, pp. 7824-7832, 2013.

[16] W. H. Hayt and J. A. Buck, Engineering electromagnetics. McGraw-Hill New York, 2001.

[17] J. B. Pendry, D. Schurig, and D. R. Smith, "Controlling Electromagnetic Fields," Science, vol. 312, no. 5781, p. 1780, 2006.

[18] S. G. Johnson and J. D. Joannopoulos, "Block-iterative frequency-domain methods for Maxwell's equations in a planewave basis," Optics Express, vol. 8, no. 3, pp. 173-190, 2001.

[19] D. W. Prather et al., "Dispersion-based optical routing in photonic crystals," Optics Letters, vol. 29, no. 1, pp. 50-52, 2004.

[20]C. R. Simovski, "Material parameters of metamaterials (a Review)," Optics and Spectroscopy, vol. 107, no. 5, p. 726, 2009.

[21]S. Arslanagić et al., "A Review of the Scattering-Parameter Extraction Method with Clarification of Ambiguity Issues in Relation to Metamaterial Homogenization," IEEE Antennas and Propagation Magazine, vol. 55, no. 2, pp. 91-106, 2013.

[22]X. Chen, T. M. Grzegorczyk, B.-I. Wu, J. Pacheco, and J. A. Kong, "Robust method to retrieve the constitutive effective parameters of metamaterials," Physical Review E, vol. 70, no. 1, p. 016608, 2004.

[23] A. B. Numan and M. S. Sharawi, "Extraction of Material Parameters for Metamaterials Using a FullWave Simulator [Education Column]," IEEE Antennas and Propagation Magazine, vol. 55, no. 5, pp. 202-211, 2013.

[24] J. H. Hannay, "The Clausius-Mossotti equation: an alternative derivation," European Journal of Physics, vol. 4, no. 3, p. 141, 1983. 
[25]E. Semouchkina, "Formation of Coherent Multi-Element Resonance States in Metamaterials," in MetamaterialCroatia: Intech, 2012.

[26]E. M. Shapiro, K. Sharer, S. Skrtic, and A. P. Koretsky, "In vivo detection of single cells by MRI," Magnetic Resonance in Medicine, vol. 55, no. 2, pp. 242-249, 2006.

[27] G. D. Merrifield et al., "Rapid and recoverable in vivo magnetic resonance imaging of the adult zebrafish at 7T," Magnetic Resonance Imaging, vol. 37, pp. 9-15, 2017.

[28] C. Baltes, N. Radzwill, S. Bosshard, D. Marek, and M. Rudin, "Micro MRI of the mouse brain using a novel $400 \mathrm{MHz}$ cryogenic quadrature RF probe," NMR in Biomedicine, vol. 22, no. 8, pp. 834-842, 2009.

[29] J. H. Duyn, "The future of ultra-high field MRI and fMRI for study of the human brain," NeuroImage, vol. 62, no. 2, pp. 1241-1248, 8/15/ 2012.

[30] J. P. Hornak, "The basics of MRI, 2008," in URL http://www. cis. rit. edu/htbooks/mri/index. html vol. 68, ed, 2008.

[31]D. E. Vincent, T. Wang, T. A. K. Magyar, P. I. Jacob, R. Buist, and M. Martin, "Birdcage volume coils and magnetic resonance imaging: a simple experiment for students," J. Biol. Eng., vol. 11, no. 1, pp. 17, Nov. 2017.

[32] J. T. Vaughan et al., "Whole-body imaging at 7T: Preliminary results," Magnetic Resonance in Medicine, vol. 61, no. 1, pp. 244-248, 2009.

[33] T. S. Ibrahim, "A Perspective into Ultra High Field MRI RF Coils," in Ultra High Field Magnetic Resonance Imaging, P.-M. Robitaille and L. Berliner, Eds. Boston, MA: Springer US, 2006, pp. 163208.

[34] T.-K. Truong, D. W. Chakeres, D. Q. Beversdorf, D. W. Scharre, and P. Schmalbrock, "Effects of static and radiofrequency magnetic field inhomogeneity in ultra-high field magnetic resonance imaging," Magnetic Resonance Imaging, vol. 24, no. 2, pp. 103-112, 2006.

[35] E. Moser, E. Laistler, F. Schmitt, and G. Kontaxis, "Ultra-High Field NMR and MRI-The Role of Magnet Technology to Increase Sensitivity and Specificity," (in English), Frontiers in Physics, vol. 5, no. 33, August 2017.

[36] A. A. Tonyushkin, J. A. Muniz, S. C. Grant, and A. J. Kiruluta, "Traveling Wave MRI at 21.1 T Propagation below Cut-off for Ultrahigh Field Vertical Bore System," arXiv:1409.1965, 2014.

[37] P. Bluem et al., "Travelling-wave excitation for 16.4T small-bore MRI," in 2015 IEEE MTT-S International Microwave Symposium, 2015, pp. 1-4, Boston, MA, USA.

[38] T. Herrmann et al., "The Travelling-Wave Primate System: A New Solution for Magnetic Resonance Imaging of Macaque Monkeys at 7 Tesla Ultra-High Field," PLOS ONE, vol. 10, no. 6, pp. 1-18, 2015.

[39] J. Hoffmann, G. Shajan, J. Budde, K. Scheffler, and R. Pohmann, "Human brain imaging at 9.4 T using a tunable patch antenna for transmission," Magnetic Resonance in Medicine, vol. 69, no. 5, pp. 1494$1500,2013$.

[40] J. T. Vaughan, "Ultra High Field MRI: High-Frequency Coils," in Ultra High Field Magnetic Resonance Imaging, P.-M. Robitaille and L. Berliner, Eds. Boston, MA: Springer US, 2006, pp. 127-161.

[41] V. C. Behr and T. Weber, Neuberger, T., Vroemen, M., Weidner, N., Bogdahn, U., Haase, A., Jakob, P. M., Faber, C., "High-resolution MR imaging of the rat spinal cord in vivo in a wide-bore magnet at 17.6 Tesla," Magnetic Resonance Materials in Physics, Biology and Medicine, vol. 17, no. 3, pp. 353-358, 2004.

[42] S. Li and J. Shen, "Integrated RF probe for in vivo multinuclear spectroscopy and functional imaging of rat brain using an 11.7 Tesla 89 mm bore vertical microimager," Magnetic Resonance Materials in Physics, Biology and Medicine, vol. 18, no. 3, pp. 119-127, 2005.

[43] A. J. E. Raaijmakers et al., "Design of a radiative surface coil array element at 7 T: The single-side adapted dipole antenna," Magnetic Resonance in Medicine, vol. 66, no. 5, pp. 1488-1497, 2011.

[44] R. Lattanzi and D. K. Sodickson, "Ideal current patterns yielding optimal snr and sar in magnetic resonance imaging: computational methods and physical insights," Magnetic Resonance in Medicine, vol. 68, no. 1, pp. 286-304, 11/29 2012.

[45] A. J. E. Raaijmakers et al., "The fractionated dipole antenna: A new antenna for body imaging at 7 Tesla," Magnetic Resonance in Medicine, vol. 75, no. 3, pp. 1366-1374, 2016.

[46] W. Lee, M. Cloos, D. Sodickson, and G. Wiggin, "Parallel transceiver array design using the modified folded dipole for 7T body applications," in in the 21st proc. ISMRM Salt Lake city, Utah, 2013, p. 292.

[47] S.-M. Hong, J. H. Park, M.-K. Woo, Y.-B. Kim, and Z.-H. Cho, "New design concept of monopole 
antenna array for UHF 7T MRI," Magnetic Resonance in Medicine, vol. 71, no. 5, pp. 1944-1952, 2014. [48] L. Alon et al., "Transverse slot antennas for high field MRI," Magnetic Resonance in Medicine, 2018.

[49] P.-M. L. Robitaille, "Ultra High Field Magnetic Resonance Imaging: A Historical Perspective," in Ultra High Field Magnetic Resonance Imaging, P.-M. Robitaille and L. Berliner, Eds. Boston, MA: Springer US, 2006, pp. 1-17.

[50] C. Qian et al., "A volume birdcage coil with an adjustable sliding tuner ring for neuroimaging in high field vertical magnets: Ex and in vivo applications at 21.1T," Journal of Magnetic Resonance, vol. 221, pp. 110-116, 2012/08/01/ 2012.

[51] T. Cheng, A. W. Magill, A. Comment, R. Gruetter, and H. Lei, "Ultra-high field birdcage coils: A comparison study at 14.1T," in 2014 36th Annual International Conference of the IEEE Engineering in Medicine and Biology Society, Chicago, IL, USA, 2014, pp. 2360-2363.

[52] T. Vaughan, "An improved volume coil for high field MRI," presented at the International Society for Magnetic Resonance in Medicine (ISMRM), Philadelphia, Pennsylvania, U.S.A, 24-28 May, 1999.

[53] S. M. Sohn, L. DelaBarre, A. Gopinath, and J. T. Vaughan, "RF Head Coil Design With Improved RF Magnetic Near-Fields Uniformity for Magnetic Resonance Imaging (MRI) Systems," IEEE Transactions on Microwave Theory and Techniques, vol. 62, no. 8, pp. 1784-1789, 2014.

[54] T. Neuberger et al., "Design of a ceramic dielectric resonator for NMR microimaging at 14.1 tesla," Concepts in Magnetic Resonance Part B: Magnetic Resonance Engineering, vol. 33B, no. 2, pp. 109114, 2008.

[55]K. Haines, T. Neuberger, M. Lanagan, E. Semouchkina, and A. G. Webb, "High Q calcium titanate cylindrical dielectric resonators for magnetic resonance microimaging," Journal of Magnetic Resonance, vol. 200, no. 2, pp. 349-353, 2009.

[56] D. O. Brunner, N. De Zanche, J. Frohlich, J. Paska, and K. P. Pruessmann, "Travelling-wave nuclear magnetic resonance," Nature, vol. 457, no. 7232, pp. 994-998, 2009.

[57] J. Hoffmann, C. Mirkes, G. Shajan, K. Scheffler, and R. Pohmann, "Combination of a multimode antenna and TIAMO for traveling-wave imaging at 9.4 Tesla," Magnetic Resonance in Medicine, vol. 75, no. 1, pp. 452-462, 2016.

[58] P. Bluem, A. Kiruluta, P. F. V. d. Moortele, A. Duh, G. Adriany, and Z. Popović, "Patch-Probe Excitation for Ultrahigh Magnetic Field Wide-Bore MRI," IEEE Transactions on Microwave Theory and Techniques, vol. 65, no. 7, pp. 2547-2557, 2017.

[59]B. Seifi, E. Semouchkina, M. Lanagan, and T. Neuberger, "Approaches to designing micro-solenoidal RF probes for 14 T MRI studies of millimeter-range sized objects," Concepts in Magnetic Resonance Part B: Magnetic Resonance Engineering, vol. 46B, no. 4, pp. 178-185, 2016, Art. no. e21349.

[60]E. Semouchkina, A. Baker, G. B. Semouchkin, M. Lanagan, and R. Mittra, "New approaches for designing microstrip filters utilizing mixed dielectrics," IEEE Transactions on Microwave Theory and Techniques, vol. 53, no. 2, pp. 644-652, 2005.

[61] A. Hennings, E. Semouchkina, A. Baker, and G. Semouchkin, "Design optimization and implementation of bandpass filters with normally fed microstrip resonators loaded by high-permittivity dielectric," IEEE Transactions on Microwave Theory and Techniques, vol. 54, no. 3, pp. 1253-1261, 2006.

[62] V. G. Veselago, "The electrodynamics of substances with simultaneously negative values of and $\mu$," Soviet physics uspekhi, vol. 10, no. 4, p. 509, 1968.

[63] D. R. Smith, W. J. Padilla, D. C. Vier, S. C. Nemat-Nasser, and S. Schultz, "Composite Medium with Simultaneously Negative Permeability and Permittivity," Physical Review Letters, vol. 84, no. 18, pp. 4184-4187, 2000.

[64] R. A. Shelby, D. R. Smith, and S. Schultz, "Experimental Verification of a Negative Index of Refraction," Science, vol. 292, no. 5514, p. 77, 2001.

[65] D. R. Smith, J. B. Pendry, and M. C. K. Wiltshire, "Metamaterials and Negative Refractive Index," Science, vol. 305, no. 5685, p. 788, 2004.

[66] J. Pendry and D. Smith, "Reversing Light: Negative Refraction.," Physics Today, vol. 57, pp. 37-43, 2004.

[67] Q. Zhao, J. Zhou, F. Zhang, and D. Lippens, "Mie resonance-based dielectric metamaterials," Materials Today, vol. 12, no. 12, pp. 60-69, 2009.

[68] D. Kajfez and P. Guillon, "Dielectric resonators," Norwood, MA, Artech House, Inc., 1986, 547 p. No individual items are abstracted in this volume., vol. 1, 1986. 
[69] O. G. Vendik and M. S. Gashinova, "Artificial double negative (DNG) media composed by two different dielectric sphere lattices embedded in a dielectric matrix," in 34th European Microwave Conference, 2004., 2004, vol. 3, pp. 1209-1212.

[70] L. Jylhä, I. Kolmakov, S. Maslovski, and S. Tretyakov, "Modeling of isotropic backward-wave materials composed of resonant spheres," Journal of Applied Physics, vol. 99, no. 4, p. 043102, 2006.

[71] T. Ueda, A. Lai, and T. Itoh, "Negative Refraction in a Cut-Off Parallel-Plate Waveguide Loaded with Two-Dimensional Lattice of Dielectric Resonators," in 2006 European Microwave Conference, 2006, pp. 435-438.

[72] E. Semouchkina, D. Werner, and C. Pantano, "An optical cloak composed of identical chalcogenide glass resonators," in 3rd International Congress on Advanced Electromagnetic Materials in Microwave and Optics, Metamateials, 2009, pp. 277-279.

[73]E. Semouchkina, D. H. Werner, G. B. Semouchkin, and C. Pantano, "An infrared invisibility cloak composed of glass," Applied Physics Letters, vol. 96, no. 23, p. 233503, 2010.

[74]H. C. Van De Hulst, Light scattering by small particles by small particles. Dover Publications Inc. : [distributor] Grantham Book Services Ltd, 1982.

[75] L. Peng, L. Ran, H. Chen, H. Zhang, J. A. Kong, and T. M. Grzegorczyk, "Experimental Observation of Left-Handed Behavior in an Array of Standard Dielectric Resonators," Physical Review Letters, vol. 98, no. 15, p. $157403,2007$.

[76] J. A. Schuller, R. Zia, T. Taubner, and M. L. Brongersma, "Dielectric Metamaterials Based on Electric and Magnetic Resonances of Silicon Carbide Particles," Physical Review Letters, vol. 99, no. 10, p. 107401,2007

[77] Y.-J. Lai, C.-K. Chen, and T.-J. Yen, "Creating negative refractive identity via single-dielectric resonators," Optics Express, vol. 17, no. 15, pp. 12960-12970, 2009.

[78] K. Vynck, D. Felbacq, E. Centeno, A. I. Căbuz, D. Cassagne, and B. Guizal, "All-Dielectric Rod-Type Metamaterials at Optical Frequencies," Physical Review Letters, vol. 102, no. 13, p. 133901, 2009.

[79]L. Peng, L. Ran, and N. A. Mortensen, "Achieving anisotropy in metamaterials made of dielectric cylindrical rods," Applied Physics Letters, vol. 96, no. 24, p. 241108, 2010.

[80] J. Wang et al., "Achieving all-dielectric left-handed metamaterials via single-sized dielectric resonators," Journal of Applied Physics, vol. 111, no. 4, p. 044903, 2012.

[81] R. Yahiaoui, U. C. Chung, C. Elissalde, M. Maglione, V. Vigneras, and P. Mounaix, "Towards left-handed metamaterials using single-size dielectric resonators: The case of TiO2-disks at millimeter wavelengths," Applied Physics Letters, vol. 101, no. 4, p. 042909, 2012.

[82] R. Yahiaoui, P. Mounaix, V. Vigneras, U. C. C. Seu, C. Elissalde, and M. Maglione, "All dielectric metamaterials at millimeter wavelengths using single-size $\mathrm{TiO} 2$ resonators: Simulation and experiments," in 2013 European Microwave Conference, 2013, pp. 1159-1162.

[83]F. Dominec, C. Kadlec, H. Němec, P. Kužel, and F. Kadlec, "Transition between metamaterial and photonic-crystal behavior in arrays of dielectric rods," Optics Express, vol. 22, no. 25, pp. 30492-30503, 2014.

[84]B. García-Cámara, F. Moreno, F. González, and O. J. F. Martin, "Light scattering by an array of electric and magnetic nanoparticles," Optics Express, vol. 18, no. 10, pp. 10001-10015, 2010.

[85] M. Nieto-Vesperinas, R. Gomez-Medina, and J. J. Saenz, "Angle-suppressed scattering and optical forces on submicrometer dielectric particles," Journal of the Optical Society of America A, vol. 28, no. 1, pp. 54-60, 2011.

[86]P. R. Wiecha et al., "Strongly Directional Scattering from Dielectric Nanowires," ACS Photonics, vol. 4, no. 8, pp. 2036-2046, 2017.

[87] M. Kerker, D. S. Wang, and C. L. Giles, "Electromagnetic scattering by magnetic spheres," Journal of the Optical Society of America, vol. 73, no. 6, pp. 765-767, 1983.

[88] M. Kerker, C. L. Giles, and D.-S. Y. Wang, "Mie scattering from a magnetic sphere immersed," J. Opt. Soc. Am., vol. 72, 1982.

[89] J. M. Geffrin et al., "Magnetic and electric coherence in forward- and back-scattered electromagnetic waves by a single dielectric subwavelength sphere," Nature Communications, Article vol. 3, p. 1171, 2012.

[90] W. Liu, A. E. Miroshnichenko, D. N. Neshev, and Y. S. Kivshar, "Broadband Unidirectional Scattering by Magneto-Electric Core-Shell Nanoparticles," ACS Nano, vol. 6, no. 6, pp. 5489-5497, 2012. 
[91] S. Person, M. Jain, Z. Lapin, J. J. Sáenz, G. Wicks, and L. Novotny, "Demonstration of Zero Optical Backscattering from Single Nanoparticles," Nano Letters, vol. 13, no. 4, pp. 1806-1809, 2013.

[92]B. S. Luk'yanchuk, N. V. Voshchinnikov, R. Paniagua-Domínguez, and A. I. Kuznetsov, "Optimum Forward Light Scattering by Spherical and Spheroidal Dielectric Nanoparticles with High Refractive Index," ACS Photonics, vol. 2, no. 7, pp. 993-999, 2015.

[93]F. Tianhua, Z. Wei, L. Zixian, and X. Yi, "Unidirectional emission in an all-dielectric nanoantenna," Journal of Physics: Condensed Matter, vol. 30, no. 12, p. 124002, 2018.

[94] J. Lv et al., "Multi-wavelength unidirectional forward scattering in the visible range in an all-dielectric silicon hollow nanodisk," Applied Optics, vol. 57, no. 17, pp. 4771-4776, 2018.

[95] Y. F. Yu, A. Y. Zhu, R. Paniagua - Domí nguez, Y. H. Fu, B. Luk'yanchuk, and A. I. Kuznetsov, "High transmission dielectric metasurface with $2 \pi$ phase control at visible wavelengths," Laser \& Photonics Reviews, vol. 9, no. 4, pp. 412-418, 2015.

[96] D. Schurig et al., "Metamaterial Electromagnetic Cloak at Microwave Frequencies," Science, vol. 314, no. 5801, p. 977, 2006.

[97]H. Chen, C. T. Chan, and P. Sheng, "Transformation optics and metamaterials," Nature Materials, Review Article vol. 9, p. 387, 2010.

[98]H. Hashemi, B. Zhang, J. D. Joannopoulos, and S. G. Johnson, "Delay-Bandwidth and Delay-Loss Limitations for Cloaking of Large Objects," Physical Review Letters, vol. 104, no. 25, p. 253903, 2010.

[99] J. Zhou, T. Koschny, M. Kafesaki, E. N. Economou, J. B. Pendry, and C. M. Soukoulis, "Saturation of the Magnetic Response of Split-Ring Resonators at Optical Frequencies," Physical Review Letters, vol. 95, no. 22, p. 223902, 2005.

[100] D. P. Gaillot, C. Croënne, and D. Lippens, "An all-dielectric route for terahertz cloaking," Optics express, vol. 16, no. 6, pp. 3986-3992, 2008.

[101] Y. A. Urzhumov and D. R. Smith, "Transformation Optics with Photonic Band Gap Media," Physical Review Letters, vol. 105, no. 16, p. 163901, 2010.

[102] D. Xiao and H. T. Johnson, "Approximate optical cloaking in an axisymmetric silicon photonic crystal structure," Optics Letters, vol. 33, no. 8, pp. 860-862, 2008.

[103] S. Jamilan, G. B. Semouchkin, N. P. Gandji, and E. Semouchkina, "Spatial dispersion of index components required for building invisibility cloak medium from photonic crystals," Journal of Optics, vol. 20 , no. 4 , p. $045102,2018$.

[104] E. Semouchkina et al., "Metamaterials-inspired miniaturization of UHF patch antennas with circular polarization," Microwave and Optical Technology Letters, vol. 53, no. 8, pp. 1938-1943, 2011.

[105] Navid P. Gandji, Akshay V. Palle, George B. Semouchkin, and E. Semouchkina, "Field-Simulation Based Engineering of RF Antenna Probes with Non-Uniform Substrates for High-Field Magnetic Resonance Imaging Systems," Applied Computational Electromagnetics Society (ACES), vol. 31, no. 5, pp. 492-497, May 2016.

[106] X. Chen, "Analytical Path to Improved RF Field Homogeneity for High Field MRI," Case Western Reserve University, 2009.

[107] F. Abboud, J. P. Damiano, and A. Papiernik, "Simple model for the input impedance of coax-fed rectangular microstrip patch antenna for CAD," IEE Proceedings $H$ - Microwaves, Antennas and Propagation, vol. 135, no. 5, pp. 323-326, 1988.

[108] M. Kazerooni, G. R. Rad, and A. Cheldavi, "Behavior Study of Simultaneously Defected Microstrip and Ground Structure (DMGS) in Planar Circuits," presented at the Progress in Electromagnetic Research Symposium Beijing, China, March 23-27, 2009.

[109] C. M. Collins and Z. Wang, "Calculation of Radiofrequency Electromagnetic Fields and Their Effects in MRI of Human Subjects," Magnetic resonance in medicine : official journal of the Society of Magnetic Resonance in Medicine / Society of Magnetic Resonance in Medicine, vol. 65, no. 5, pp. 14701482, 2011.

[110] R. Pethig, "Dielectric properties of body tissues," Clinical Physics and Physiological Measurement, vol. 8, no. 4A, p. 5, 1987.

[111] K. Carver and J. Mink, "Microstrip antenna technology," IEEE Transactions on Antennas and Propagation, vol. 29, no. 1, pp. 2-24, 1981.

[112] J. R. James, P. S. Hall, and C. Wood, Microstrip antenna: Theory and design (no. 12). Iet, 1981. 
[113] A. B. Constantine, "Antenna theory: analysis and design," MICROSTRIP ANTENNAS, third edition, John wiley \& sons, 2005.

[114] E. Semouchkina, R. Duan, G. Semouchkin, and R. Pandey, "Sensing Based on Fano-Type Resonance Response of All-Dielectric Metamaterials," Sensors, vol. 15, no. 4, 2015.

[115] N. P. Gandji, G. B. Semouchkin, and E. Semouchkina, "All-dielectric metamaterials: irrelevance of negative refraction to overlapped Mie resonances," Journal of Physics D: Applied Physics, vol. 50, no. 45, p. 455104, 2017.

[116] T. Koschny, P. Markoš, D. R. Smith, and C. M. Soukoulis, "Resonant and antiresonant frequency dependence of the effective parameters of metamaterials," Physical Review E, vol. 68, no. 6, p. 065602, 2003.

[117] T. Koschny, P. Markoš, E. N. Economou, D. R. Smith, D. C. Vier, and C. M. Soukoulis, "Impact of inherent periodic structure on effective medium description of left-handed and related metamaterials," Physical Review B, vol. 71, no. 24, p. 245105, 2005.

[118] C. Vandenbem and J. P. Vigneron, "Mie resonances of dielectric spheres in face-centered cubic photonic crystals," Journal of the Optical Society of America A, vol. 22, no. 6, pp. 1042-1047, 2005.

[119] N. Liu and H. Giessen, "Coupling Effects in Optical Metamaterials," Angewandte Chemie International Edition, vol. 49, no. 51, pp. 9838-9852, 2010.

[120] P. Moitra, Y. Yang, Z. Anderson, I. I. Kravchenko, D. P. Briggs, and J. Valentine, "Realization of an all-dielectric zero-index optical metamaterial," Nat Photon, Letter vol. 7, no. 10, pp. 791-795, 2013.

[121] P. V. Parimi, W. T. Lu, P. Vodo, J. Sokoloff, J. S. Derov, and S. Sridhar, "Negative Refraction and Left-Handed Electromagnetism in Microwave Photonic Crystals," Physical Review Letters, vol. 92, no. 12, p. 127401, 2004.

[122] A. Hosseinzadeh and E. Semouchkina, "Effect of permittivity on energy band diagrams of dielectric metamaterial arrays," Microwave and Optical Technology Letters, vol. 55, no. 1, pp. 134-137, 2013.

[123] E. Semouchkina, R. Duan, N. P. Gandji, S. Jamilan, G. B. Semouchkin, and R. Pandey, "Superluminal media formed by photonic crystals for transformation optics-based invisibility cloaks," Journal of Optics, vol. 18, no. 4, p. 044007, 2016.

[124] R. C. Rumpf and J. Pazos, "Synthesis of spatially variant lattices," Optics Express, vol. 20, no. 14, pp. 15263-15274, 2012.

[125] H. Kosaka et al., "Self-collimating phenomena in photonic crystals," Applied Physics Letters, vol. 74, no. 9, pp. 1212-1214, 1999.

[126] D. W. Prather et al., "Self-collimation in photonic crystal structures: a new paradigm for applications and device development," Journal of Physics D: Applied Physics, vol. 40, no. 9, p. 2635, 2007.

[127] S. Shi, A. Sharkawy, C. Chen, D. M. Pustai, and D. W. Prather, "Dispersion-based beam splitter in photonic crystals," Optics Letters, vol. 29, no. 6, pp. 617-619, 2004.

[128] B. Miao, C. Chen, A. Sharkway, S. Shi, and D. W. Prather, "Two bit optical analog-to-digital converter based on photonic crystals," Optics Express, vol. 14, no. 17, pp. 7966-7973, 2006.

[129] G. Magno et al., "Controlled reflectivities in self-collimating mesoscopic photonic crystal," Journal of the Optical Society of America B, vol. 31, no. 2, pp. 355-359, 2014.

[130] H. Alipour-Banaei, M. G. Rabati, P. Abdollahzadeh-Badelbou, and F. Mehdizadeh, "Application of self-collimated beams to realization of all optical photonic crystal encoder," Physica E: Low-dimensional Systems and Nanostructures, vol. 75, pp. 77-85, 2016.

[131] W. Park, "Controlling the flow of light with silicon nanostructures," Laser Physics Letters, vol. 7, no. 2 , p. 93, 2010.

[132] N. P. Gandji and E. Semouchkina, "Employing self-collimation phenomena in photonic crystals for the invisibility cloak development," in 2016 IEEE International Symposium on Antennas and Propagation (APSURSI), 2016, pp. 1967-1968. 


\section{Appendix A. Copyright permission of the figures in chapter 3}

\section{Order Details}

Journal of Physics D : Applied Physics

\begin{tabular}{|c|c|c|}
\hline & & $\begin{array}{l}\text { Billing Status: } \\
\text { N/A }\end{array}$ \\
\hline \multicolumn{3}{|c|}{ Permission Status: $\mho_{\text {Granted }}$} \\
\hline \multicolumn{3}{|c|}{$\begin{array}{ll}\text { Permission type: } & \text { Republish or display content } \\
\text { Type of use: } & \text { Thesis/Dissertation } \\
& \text { Order License Id: } 4385660539775\end{array}$} \\
\hline \multicolumn{3}{|l|}{ Hide details } \\
\hline Requestor type & \multicolumn{2}{|c|}{$\begin{array}{l}\text { Author of requested } \\
\text { content }\end{array}$} \\
\hline Format & \multicolumn{2}{|c|}{ Electronic } \\
\hline Portion & \multicolumn{2}{|c|}{ chart/graph/table/figure } \\
\hline $\begin{array}{l}\text { Number of } \\
\text { charts/graphs/tables/f } \\
\text { igures }\end{array}$ & \multicolumn{2}{|c|}{ 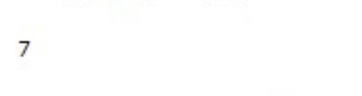 } \\
\hline $\begin{array}{l}\text { The requesting } \\
\text { person/organization }\end{array}$ & \multicolumn{2}{|c|}{ Navid Pourramzan Gandji } \\
\hline $\begin{array}{l}\text { Title or numeric } \\
\text { reference of the } \\
\text { portion(s) }\end{array}$ & \multicolumn{2}{|c|}{$\begin{array}{l}\text { Figure } 3.1 \text {, Figure } 3.2, \\
\text { Figure } 3.3 \text {, Figure } 3.4, \\
\text { Figure } 3.5 \text {, Figure } 3.6, \\
\text { Figure } 3.7 \text {, Figure } 3.8, \\
\text { Figure } 3.9\end{array}$} \\
\hline $\begin{array}{l}\text { Title of the article or } \\
\text { chapter the portion is } \\
\text { from }\end{array}$ & \multicolumn{2}{|c|}{$\begin{array}{l}\text { Chapter } 3 \text {. Clarifying the } \\
\text { nature of negative } \\
\text { refraction in all-dielectric } \\
\text { metamaterials composed } \\
\text { of identical resonators }\end{array}$} \\
\hline Editor of portion(s) & \multicolumn{2}{|c|}{ N/A } \\
\hline Author of portion(s) & \multicolumn{2}{|c|}{ Navid P.Gandji } \\
\hline $\begin{array}{l}\text { Volume of serial or } \\
\text { monograph }\end{array}$ & \multicolumn{2}{|c|}{$\mathrm{N} / \mathrm{A}$} \\
\hline Page range of portion & \multicolumn{2}{|l|}{$38-48$} \\
\hline $\begin{array}{l}\text { Publication date of } \\
\text { portion }\end{array}$ & \multicolumn{2}{|l|}{ July 2018} \\
\hline Rights for & \multicolumn{2}{|c|}{ Main product } \\
\hline Duration of use & \multicolumn{2}{|c|}{ Life of current edition } \\
\hline $\begin{array}{l}\text { Creation of copies for } \\
\text { the disabled }\end{array}$ & \multicolumn{2}{|c|}{ yes } \\
\hline $\begin{array}{l}\text { With minor editing } \\
\text { privileges }\end{array}$ & \multicolumn{2}{|l|}{ yes } \\
\hline For distribution to & \multicolumn{2}{|c|}{ Worldwide } \\
\hline $\begin{array}{l}\text { In the following } \\
\text { language(s) }\end{array}$ & \multicolumn{2}{|c|}{$\begin{array}{l}\text { Original language of } \\
\text { publication }\end{array}$} \\
\hline $\begin{array}{l}\text { With incidental } \\
\text { promotional use }\end{array}$ & \multicolumn{2}{|l|}{ no } \\
\hline $\begin{array}{l}\text { Lifetime unit quantity of } \\
\text { new product }\end{array}$ & \multicolumn{2}{|c|}{ Up to 499} \\
\hline Title & \multicolumn{2}{|c|}{$\begin{array}{l}\text { EMPLOYING DIELECTRIC- } \\
\text { BASED MEDIA FOR } \\
\text { CONTROLLING FIELD } \\
\text { PATTERNS AND WAVE } \\
\text { PROPAGATION IN } \\
\text { ADVANCED }\end{array}$} \\
\hline Instructor name & \multicolumn{2}{|c|}{ Elena Semouchkina } \\
\hline Institution name & \multicolumn{2}{|c|}{$\begin{array}{l}\text { Michigan Technological } \\
\text { University }\end{array}$} \\
\hline $\begin{array}{l}\text { Expected presentation } \\
\text { date }\end{array}$ & \multicolumn{2}{|l|}{ Jul 2018} \\
\hline
\end{tabular}




\section{Appendix B. Copyright permission of the figures 5.4 and 5.5}

\section{Order Details}

Journal of Optics
Billing Status:

\begin{tabular}{|c|c|c|}
\hline & & $\begin{array}{c}\text { Billing Status: } \\
\text { N/A }\end{array}$ \\
\hline \multicolumn{3}{|c|}{ Permission Status: $\checkmark$ Granted } \\
\hline $\begin{array}{l}\text { Permission type: } \\
\text { Type of use: }\end{array}$ & $\begin{array}{l}\text { or display } \\
\text { sertation } \\
\text { ense Id: }\end{array}$ & 981056420 \\
\hline \multicolumn{3}{|l|}{$\square$ Hide details } \\
\hline Requestor type & $\begin{array}{l}\text { Author of } \\
\text { content }\end{array}$ & sted \\
\hline Format & Print, Elec & \\
\hline Portion & chart/grap & ole/figure \\
\hline $\begin{array}{l}\text { Number of } \\
\text { charts/graphs/tables/f } \\
\text { igures }\end{array}$ & 2 & \\
\hline $\begin{array}{l}\text { The requesting } \\
\text { person/organization }\end{array}$ & \multicolumn{2}{|c|}{ Navid Pourramzan Gandji } \\
\hline $\begin{array}{l}\text { Title or numeric } \\
\text { reference of the } \\
\text { portion(s) }\end{array}$ & \multicolumn{2}{|c|}{ Figure 5.4, Figure 5.5} \\
\hline $\begin{array}{l}\text { Title of the article or } \\
\text { chapter the portion is } \\
\text { from }\end{array}$ & \multicolumn{2}{|c|}{$\begin{array}{l}\text { Chapter } 5 \text {. Selected } \\
\text { problems of implementing } \\
\text { all-dielectric photonic } \\
\text { crystals in the media of } \\
\text { transformation optics } \\
\text { based devices }\end{array}$} \\
\hline Editor of portion(s) & \multicolumn{2}{|l|}{ N/A } \\
\hline Author of portion(s) & \multicolumn{2}{|c|}{ Navid P. Gandji } \\
\hline $\begin{array}{l}\text { Volume of serial or } \\
\text { monograph }\end{array}$ & \multicolumn{2}{|l|}{$\mathrm{N} / \mathrm{A}$} \\
\hline Page range of portion & \multicolumn{2}{|l|}{$60-71$} \\
\hline $\begin{array}{l}\text { Publication date of } \\
\text { portion }\end{array}$ & \multicolumn{2}{|l|}{ Aug 2018} \\
\hline Rights for & \multicolumn{2}{|c|}{ Main product } \\
\hline Duration of use & \multicolumn{2}{|c|}{ Life of current edition } \\
\hline $\begin{array}{l}\text { Creation of copies for } \\
\text { the disabled }\end{array}$ & \multicolumn{2}{|l|}{ yes } \\
\hline $\begin{array}{l}\text { With minor editing } \\
\text { privileges }\end{array}$ & \multicolumn{2}{|l|}{ yes } \\
\hline For distribution to & \multicolumn{2}{|c|}{ Worldwide } \\
\hline $\begin{array}{l}\text { In the following } \\
\text { language(s) }\end{array}$ & \multicolumn{2}{|c|}{$\begin{array}{l}\text { Original language of } \\
\text { publication }\end{array}$} \\
\hline $\begin{array}{l}\text { With incidental } \\
\text { promotional use }\end{array}$ & \multicolumn{2}{|l|}{ no } \\
\hline $\begin{array}{l}\text { Lifetime unit quantity of } \\
\text { new product }\end{array}$ & \multicolumn{2}{|l|}{ Up to 499} \\
\hline Title & \multicolumn{2}{|c|}{$\begin{array}{l}\text { EMPLOYING DIELECTRIC- } \\
\text { BASED MEDIA FOR } \\
\text { CONTROLLING FIELD } \\
\text { PATTERNS AND WAVE } \\
\text { PROPAGATION IN } \\
\text { ADVANCED }\end{array}$} \\
\hline Instructor name & \multicolumn{2}{|c|}{ Elena Semouchkina } \\
\hline Institution name & \multicolumn{2}{|c|}{$\begin{array}{l}\text { Michigan Technological } \\
\text { University }\end{array}$} \\
\hline $\begin{array}{l}\text { Expected presentation } \\
\text { date }\end{array}$ & \multicolumn{2}{|l|}{ Jul 2018} \\
\hline
\end{tabular}

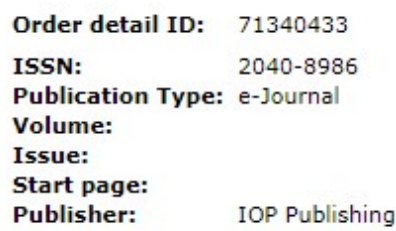




\section{Appendix C. Copyright permission of the figures 5.6 and 5.7}

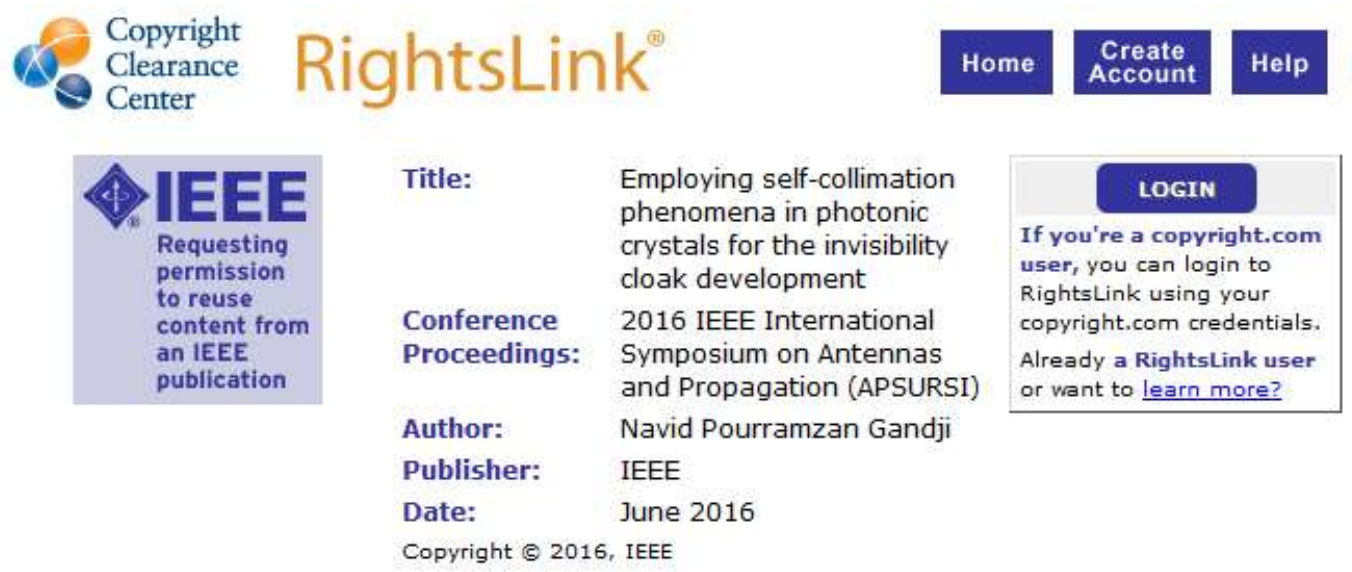

Thesis / Dissertation Reuse

The IEEE does not require individuals working on a thesis to obtain a formal reuse license, however, you may print out this statement to be used as a permission grant:

Requirements to be followed when using any portion (e.g., figure, graph, table, or textual material) of an IEEE copyrighted paper in a thesis:

1) In the case of textual material (e.g., using short quotes or referring to the work within these papers) users must give full credit to the original source (author, paper, publication) followed by the IEEE copyright line (c) 2011 IEEE.

2) In the case of illustrations or tabular material, we require that the copyright line (c) [Year of original publication] IEEE appear prominently with each reprinted figure and/or table.

3) If a substantial portion of the original paper is to be used, and if you are not the senior author, also obtain the senior author's approval.

Requirements to be followed when using an entire IEEE copyrighted paper in a thesis:

1) The following IEEE copyright/ credit notice should be placed prominently in the references: (c) [year of original publication] IEEE. Reprinted, with permission, from [author names, paper title, IEEE publication title, and month/year of publication]

2) Only the accepted version of an IEEE copyrighted paper can be used when posting the paper or your thesis on-line.

3) In placing the thesis on the author's university website, please display the following message in a prominent place on the website: In reference to IEEE copyrighted material which is used with permission in this thesis, the IEEE does not endorse any of [university/educational entity's name goes here]'s products or services. Internal or personal use of this material is permitted. If interested in reprinting/republishing IEEE copyrighted material for advertising or promotional purposes or for creating new collective works for resale or redistribution, please go to http://www.ieee.org/publications standards/publications/riqhts /riahts link.html to learn how to obtain a License from RightsLink.

If applicable, University Microfilms and/or ProQuest Library, or the Archives of Canada may supply single copies of the dissertation. 